\title{
Avifauna (Non Passeriformes) of Santa Cruz province, Patagonia (Argentina): annotated list of species
}

\author{
Carlos Aquiles DARRIEU ${ }^{1}$, Aníbal Raúl CAMPERI ${ }^{1,2} \&$ Santiago IMBERTI $^{3}$ \\ ${ }^{1}$ División Zoología Vertebrados, Sección Ornitología, Museo de La Plata, Paseo del Bosque, 1900 La Plata, Pcia. \\ de Buenos Aires, cdarrieu@fcnym.unlp.edu.ar. ${ }^{2}$ Adscripto a la División Ornitología, Museo Argentino de \\ Ciencias Naturales "Bernardino Rivadavia", Av. A. Gallardo 470, C1405DJR Buenos Aires, \\ acamperi@fcnym.unlp.edu.ar. ${ }^{3}$ Rivadavia 780,9400 Río Gallegos, Argentina, imbertis@ar.inter.net
}

\begin{abstract}
The birds of Santa Cruz Province, Argentina, are analyzed based on three main sources: specimens housed in the Museo Argentino de Ciencias Naturales of Buenos Aires, in the Félix de Azara Collection, Buenos Aires (CFA), in the Museo de La Plata, La Plata, in the Fundación Miguel Lillo, Tucumán and in the National Museum of Natural History (USNM); from bibliographical citation which include precise localities, and from field observations. A list of 150 species belonging to 33 families is presented. First records with precise localities are provided for nine species. New localities are cited for 114 species.
\end{abstract}

Key words: Birds, systematic list, distribution, Santa Cruz, Argentina.

Resumen: En el presente trabajo se aporta una lista comentada de todas las especies de aves no Passeriformes registradas en la provincia de Santa Cruz. Los datos fueron obtenidos de la literatura, del análisis de especímenes de museos y de observaciones de campo realizadas por uno de los autores. Esto nos permitió incluir un total de 150 especies. Nueve de las mismas no presentaban registros concretos para la provincia, aportándose nuevas localidades para otras 114. Los ejemplares de colección pertenecen al Museo Argentino de Ciencias Naturales y a la Colección Félix de Azara, ambos de Buenos Aires, al Museo de La Plata, y a la Fundación Miguel Lillo de Tucumán y al National Museum of Natural history (USNM).

Palabras Clave: Aves, lista sistemática, distribución, Santa Cruz, Argentina.

\section{INTRODUCCION}

Although an important number of papers relating to different aspects of the ornithological fauna of Santa Cruz exist, all of them refer to particular areas or singular species that have been studied from a systematic or biological point of view. Among them it is possible to mention Zapata (1967, 1969), Daciuk (1977), De Lucca \& Saggese (1992), Gandini et al. (1997), Frere \& Gandini (1998), Gandini \& Frere (1998), Yorio (1998), Yorio et al. (1998), Nasca et al. (2004) and Imberti (2005). This contribution is the first list that makes a complete revision of the occurrence of each species in the Santa Cruz province. It was compiled according to bibliographic data, skins from different museum collections and field observations.

A number of 150 species belonging to 33 families of Non Passeriformes were registred.

New localities on the basis of collected material and field observations for 43 species are given. 266 localities (6 not localized) are mentioned and pin-pointed on the map (Fig. 1).

\section{Study area}

The province of Santa Cruz is the second largest of Argentina (after Buenos Aires) with an area of $243.943 \mathrm{~km}^{2}$. Two distinct zones were recognized whitin this area. The first one belongs to the Andean region located in its western part, which is characterized by the presence of a section of the Andes System called Patagonian Andes. The second one belongs to the Extra-andean region which extends over the central and eastern part of the province, and is characterized by terraced plateaus decreasing in altitude towards the East, reaching the sea with 300 meters high cliffs and broad beaches. At the foothills of the Andean System glacial lakes known as Argentino, Viedma, Buenos Aires and San Martín are nourished by glaciers coming from the Patagonian Ice Fields.

Biogeographically most of Santa Cruz belongs to the Patagonian Province. It is characterized by rocky-sandy soils and arid weather with low temperatures during most of the year, strong thermal amplitude and little precipitation, setting a semi-desert biome. The dominant flora is a shrubby steppe with leafless bushes, some with 


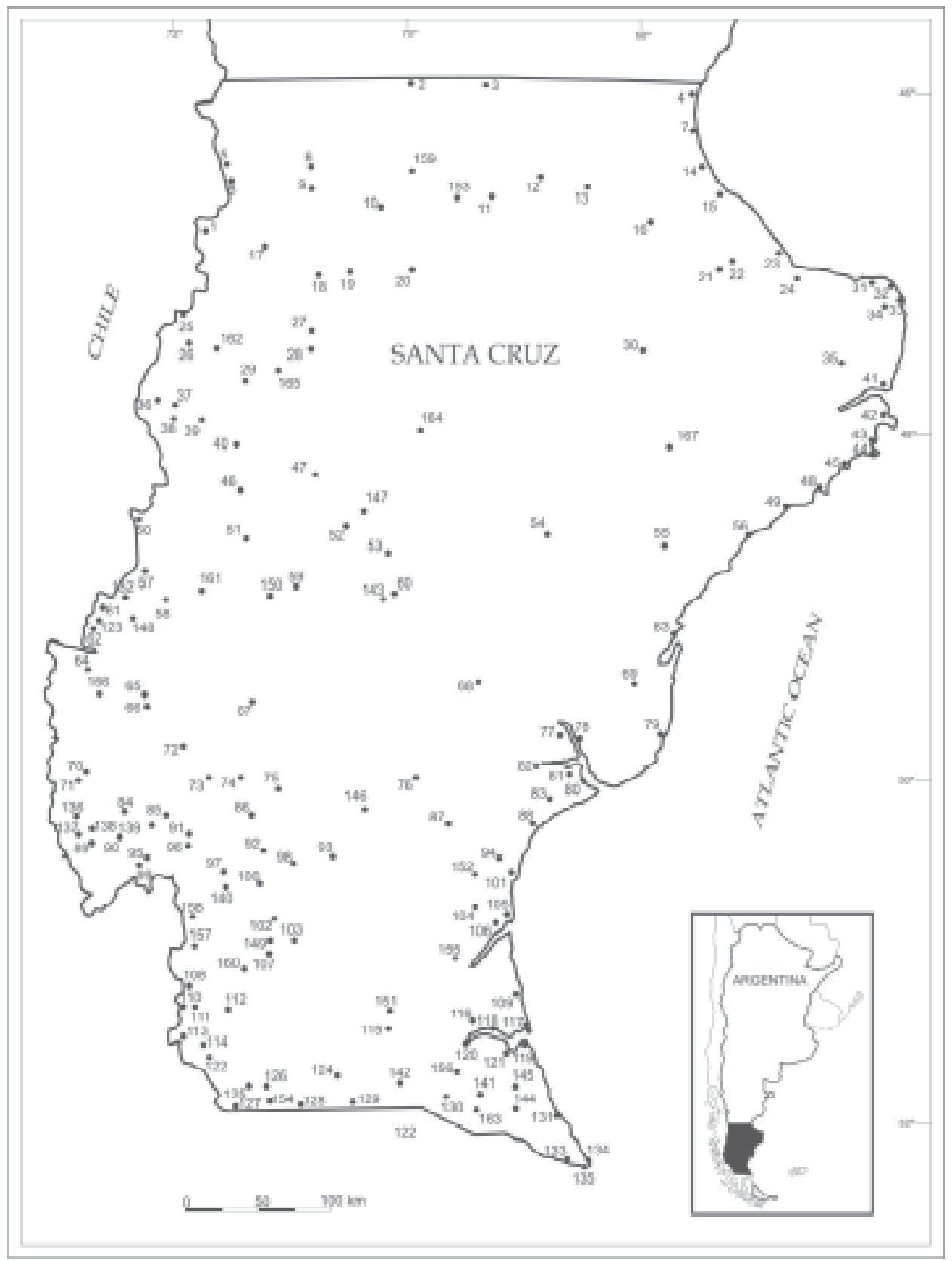

Fig. 1: Map showing the localities cited in the text (arranged in alphabetical order).

reduced or thorny leaves or with the predominance of cushion shaped plants. Overgrazed by cattle, grass and herbaceous dicotiledons grow between the bushes. To a lesser extent there is a narrow western strip of land representing the Sub Antarctic Province that occupies mountains and transverse glacial valleys. The weather is temperate and humid with a mean temperature of $7^{\circ} \mathrm{C}$.
Rains reach more than $2000 \mathrm{~mm}$ along the Argentina-Chile border diminishing rapidly eastwards to up to 700 or 800 annual $\mathrm{mm}$. The dominant flora is the deciduous or perennial forest but there are some grasslands, high Andean peatbogs, etc. Finally, the High Andes Province, is represented by the Austral High Andes District that occupies the mountain slopes at approximately 
1000 meters above sea level. It is distinguished by rocky or sandy immature soils, a cold and dry high mountain climate and rains in form of hail or snow at anytime of the year. This District runs discontinuously forming islands over the Sub Antarctic Province, enriching the High Andean flora with subantarctic elements. The communities represented are grassy steppes, high Andean peatbogs, and Pampas grass consociations, etc. (Cabrera, 1971; Cabrera \& Willink, 1973).

\section{MATERIALS AND METHODS}

A revision of the ornithological collection from the province of Santa Cruz, stored in the Museo Argentino de Ciencias Naturales, Buenos Aires (MACN), the Félix de Azara Collection, Buenos Aires (CFA), the Museo de La Plata, Buenos Aires (MLP), the Fundación Miguel Lillo, Tucumán (FML) and the National Museum of Natural History, Washington (USNM) is provided in the present work.

The taxonomy follows Del Hoyo et al. (1992, 1994, 1996, 1997, 1999, 2001 and 2002) and the systematic sequence follows Mazar Barnett \& Pearman (2001). Locality, number of individuals, sex (m: male; f: female), date of capture, collector and place of storage are given for the specimens in the collections mentioned above.

Species not found in the collections but cited by other authors are included as well as observations made by the authors and other specialists. Subspecific assignment was made according to geographic distribution in the cases where specimens were not available. Only the sea birds species detected in the coastal area are included, thus the species detected in the Argentinian Sea are excluded in the current list.

Localities are ordered alphabetically with a number assigned for each location on the map in Appendix 1. The catalogue of Paynter Jr. (1995) and a Geografic Atlas of the Argentine Republic edited by the Centro Cartográfico Buenos Aires SRL were used to locate each collecting localitiy. Localities are numbered from north to south on the map (Fig. 1).

\section{ANNOTATED CHECKLIST}

\section{RHEIDAE}

\section{Rhea pennata pennata}

Literature. Puerto Deseado (Gould, 1837b; Zapata, 1967); Cañadón Misioneros, Cerro de la Picana (Oustalet, 1891); Ría Coig (Scott \& Sharpe, 1904); Lago San Martín (Wetmore, 1926a); between Mazarredo and Cabo Blanco (Zapata, 1969);
Laguna Los Escarchados (Lange, 1981); Estancia El Cuadro, Monumento Natural Bosques Petrificados (De Lucca \& Saggese, 1992); Parque Nacional Perito Moreno, Tamel Aike, Tres Lagos, El Chaltén, El Calafate, Cerrito, Tapi Aike, Cancha Carrera, Monumento Nacional Bosques Petrificados, Río Pinturas, Parque Nacional Los Glaciares, La Esperanza, Lago Cardiel Comandante Luis Piedra Buena, Gobernador Gregores (de la Peña, 2005); Parque Nacional Los Glaciares (Bahía del Túnel, Lago Viedma, El Chaltén, Lago Roca) (Imberti, 2005); Estancia La Angostura (Imberti, unpubl. b); Río Gallegos, Río Coig, Meseta Lago Strobel, Meseta del Asador, Río Chico, Meseta Lago Buenos Aires, Estancia El Cóndor, El Zurdo, Parque Nacional Los Glaciares (Coconier, 2005); Cabo Vírgenes, Monte León, Río Gallegos, Río Coig, Meseta Lago Strobel, Río Chico, Monumento Natural Bosques Petrificados, Estancia El Cuadro, Parque Nacional Perito Moreno, Meseta Lago Buenos Aires, El Zurdo, Laguna Nimez, Lago Argentino, Meseta del Asador, Parque Nacional Los Glaciares, Estancia La Soledad, Estancia La Anita, Estancia El Cóndor, Bahía San Julián (Di Giácomo et al., 2005).

Museum specimens. Isla Pingüino, 1 f, 17 Jan 1916, S. Shipton; Las Heras, Estancia Roca Blanca, 1 m, 1 Jan 1961, O. Budin, J.C. Viera, FML; Puerto Coig, a brood, May 1974, col. M. Rumboll, MACN.

Field observations. Monumento Natural Bosques Petrificados, Río Gallegos, Cabo Vírgenes, Estancia Rincón de los Morros, Estancia Verdadera Argentina, Parque Nacional Perito Moreno, Parque Nacional Los Glaciares, Estancia Rupai Pacha, Laguna Los Escarchados, Estancia Pali Aike, Laguna Azul, Ría Coig, Estancia Bella Vista Bitsch, Estancia Cabo Buen Tiempo, Monte León, Río Coig, El Zurdo, Estancia El Cóndor, Estancia La Carlota, Estancia Sofía, Estancia Killik Aike Sur, Estancia Coy Inlet, El Roda, Estancia Bella Vista (Imberti).

\section{TINAMIDAE}

\section{Eudromia elegans patagonica}

Literature. Aguada Grande (Dabbene, 1923); Bahía del Fondo (Steullet \& Deautier, 1935); Pampa Alta (Hellmayr \& Conover, 1942; Conover, 1950); Puerto Deseado (Zapata, 1967); Caleta Olivia (Zapata, 1969); Gobernador Gregores, Lago Argentino, Perito Moreno, Santa Cruz (Bohl, 1970); Estancia El Cuadro, Monumento Natural Bosques Petrificados (De Lucca \& Saggese, 1992); Bahía del Fondo, Aguada Grande (Navas \& Bó, 1981); Lago Viedma (Imberti, 2005); Río Coig, Río Chico, Monumento Nacional Bosques Petri- 
ficados, Estancia El Cuadro, Laguna Nimez, Lago Argentino, Bahía San Julián (Di Giácomo et al., 2005); Estancia La Angostura (Imberti, unpubl. b). Museum specimens. Bahía del Fondo, $1 \mathrm{~m}, 14$ Dec 1921, col. A. Merkle, MLP; Las Heras, Estancia El Tranquilo, Estancia Roca Blanca, 3 ?, 24 and 27 Jan 1961, col. O. Budin and J.C. Viera; Puerto Deseado, 2 ?, 1 Jan 1915, 5 Jan 1916, col. S. Shipton, FML.

Field observations. Monumento Natural Bosques Petrificados, Ría Coig, Estancia Moy Aike Grande, Estancia La Carreta, Estancia Las Horquetas (Imberti).

\section{Tinamotis ingoufi}

Literature. Santa Cruz (Oustalet, 1890, 1891); San Julián (Dabbene, 1920b); Barranca Blanca, Aguada Grande, Río Coig, mouth of Río Santa Cruz (Pozzi, 1923); Aguada Grande (Liebermann, 1936); Aguada Grande, Pampa Alta (Hellmayr \& Conover, 1942); Estancia El Cuadro (De Lucca \& Saggese, 1992); Perito Moreno (45 km south of), La Manchuria, Estancia Coy Inlet, Estancia La Angostura, Monumento Nacional Bosques Petrificados, Bahía Laura, Estancia Cañadón Vasco, Cañadón de las Vacas, Reserva Provincial Península San Julián (Imberti, 2003); Gobernador Gregores (de la Peña, 2005); Parque Nacional Los Glaciares (Sección Centro, Lago Viedma, Lago Argentino) (Imberti, 2005); Río Coig, Meseta Lago Strobel, Río Chico, Meseta Lago Buenos Aires, Laguna Nimez, Meseta del Asador (Coconier, 2005); Río Coig, Meseta Lago Strobel, Río Chico, Monumento Natural Bosques Petrificados, Estancia El Cuadro, Meseta Lago Buenos Aires, Laguna Nimez, Lago Argentino, Meseta del Asador, Parque Nacional Los Glaciares, Estancia La Soledad, Estancia La Anita, Bahía San Julián (Di Giácomo et al., 2005); Cabo Vírgenes (Imberti, unpubl. a)

Museum specimens. Aguada Grande, 1 ?, 29 Apr 1923, col. A. Pozzi, MACN; 1 f, Apr 1923, col. A. Pozzi, USNM; Bahía Laura, 1 ?, muscular tissue, 10 Jan 2001, col. S. Imberti, MACN; Cerro Mojón (450 m.a.s.l.), Estancia Reconquista, 1 ?, 24 Jan 1961, col. O. Budin and J.C. Viera; Estancia El Tranquilo, $60 \mathrm{~km}$ to Bosques Petrificados, 1 ?, 15 Feb 1976, col. C. Olrog, FML; Gobernador Gregores, $2 \mathrm{~m}$ and $1 \mathrm{f}$, Mar and Apr 1973, col. Watley, MLP; Perito Moreno, 1 ?, skeleton, 15 Jan 1991, col. L.M. Chiappe; San Julián (60 km south), 1 m, 5 May 1974, col. M. Rumboll and E. Shaw, MACN; Tellier, Puerto Deseado, 1 ?, 20 Sep1962, col. N. Magaldi, MLP; Tres Lagos, $1 \mathrm{~m}$ and 1 ?, skeleton, 15 Jan 1991, col. L.M. Chiappe, MACN. Field observations. Monumento Natural Bosques Petrificados, Perito Moreno, Ría Coig,
Bahía Laura, Estancia Cañadón Vasco, Laguna Nimez (Imberti); Río Bote (Gardner pers. comm.); Estancia La Angostura (Rodríguez Goñi pers. comm.).

\section{SPHENISCIDAE}

\section{Aptenodytes patagonicus patagonicus}

Field observations. Cabo Vírgenes (Fenton pers. comm.)

\section{Pygoscelis papua ssp.}

Literature. Puerto Deseado (Olrog, 1973; Daciuk, 1976).

Field observations. Cabo Vírgenes (Sturzenbaum pers. comm.)

\section{Eudyptes chrysocome chrysocome}

Literature. Puerto Deseado (Zapata, 1967; Gandini \& Frere, 1998b); Cabo Blanco (Zapata, 1969); Isla Pingüino, $35 \mathrm{~km}$ from Puerto Deseado (Frere et al., 1993; Yorio, 1998; Schiavini et al., 2005); Bahía Oso Marino (Gandini \& Frere, 1998a); Río Deseado (Di Giácomo et al., 2005; Coconier, 2005).

Museum specimens. Puerto Deseado, $1 \mathrm{~m}, 17$ Jan 1916, collector unknown, MACN.

Field observations. Isla Pingüino, Ría Deseado, Punta Loyola, Ría Gallegos (Imberti).

\section{Eudyptes chrysolophus}

Literature. Puerto Deseado (Olrog, 1973).

Field observations. Isla Pingüino, Santa Cruz (Harris pers. comm.)

\section{Spheniscus magellanicus}

Literature. Islas Leones (Salvadori, 1900; Hartert \& Venturi, 1909); Puerto Deseado (Doello Jurado, 1917; Daneri, 1959; Zapata, 1967; Olrog, 1973; Daciuk, 1977; Gandini \& Frere, 1998b), Nasca et al., 2004); Ría Deseado (Renard, 1931; Olrog, 1975; Albrieu \& Navarro, 1997); San Julián (Anónimo, 1933); Bahía del Fondo (Steullet \& Deautier, 1935); Punta Medanosa (Birabén \& Hylton Scott, 1939); Isla Leones, Puerto San Julián, Puerto Deseado, Río Santa Cruz, Bahía Laura (Carrara, 1952); Caleta Olivia, Cabo Blanco (Zapata, 1969); Cabo Vírgenes, Río Gallegos, Monte León, Ría Santa Cruz, Ría San Julián, Bahía Laura, Ría Deseado, Isla de los Pájaros (Daciuk, 1976a \& b); Cabo Vírgenes (Lucero, 1982; Gandini et al., 1992, 1997; Frere et al., 1998; Cruz et al., 2005); Punta Dungeness, Puerto Deseado (de la Peña, 1987, 2005); between Cabo Vírgenes and Punta Dungeness (Goodall et al., 1991); between Cabo Vírgenes, Punta Dungeness, Caleta Olivia (Frere et al., 1996); Isla Chaffers, Isla 
Pingüino, Bahía Laura, Cabo Vírgenes (Frere et al., 1996); Ría Deseado, Bahía Oso Marino, Punta Medanosa, Bahía Laura, Bahía San Julián, Ría Santa Cruz, Monte León, Río Gallegos, Cabo Vírgenes (Yorio, 1998); Punta de los Pájaros, Río Deseado, Bahía Oso Marino, Punta Buque (Gandini \& Frere, 1998a; Schiavini et al., 2005); Bahía Laura, Bahía San Julián, Ría Santa Cruz, Monte León, Isla Deseada, Cabo Vírgenes (Frere \& Gandini, 1998); Bahía San Julián (Wilson et al., 2001); Isla de los Pájaros, Isla Quiroga (Cevasco et al., 2001); Puerto Deseado, San Julián, Cabo Vírgenes (Wilson et al., 2005); Cabo Vírgenes, Río Deseado, Monte León, Río Gallegos, Isla Deseada, Río Coig (Coconier, 2005); Cabo Vírgenes, Río Deseado, Monte León, Río Gallegos, Río Coig, Bahía San Julián (Di Giácomo et al., 2005).

Museum specimens. Puerto Deseado, $1 \mathrm{~m}$ and 1 ?, 19 Dec 1915, S. Shipton, FML;1 young, 13 Dec 1941, col. J. Pereyra, MACN; 2 ?, 16 Nov 1961, col. A. Zapata, MACN.

Field observations. Isla Pingüino, Isla de los Pájaros, Monte León, Isla Deseada, Cabo Vírgenes, Punta Loyola, Ría Coig, Estancia Cabo Buen Tiempo, Estancia El Cóndor, Punta Quilla, Bahía San Julián (Imberti).

\section{PODICIPEDIDAE}

\section{Rollandia rolland chilensis}

Literature. Río Gallegos (Oustalet, 1891; Hellmayr \& Conover, 1948a); Cabo Buen Tiempo, Río Gallegos, Río Ecker (Scott \& Sharpe, 1904); Laguna Los Escarchados (Lange, 1981); Reserva de Vida Silvestre Los Escarchados (Erize, 1983); Laguna Nimez (Imberti \& Albrieu, 2001); Parque Nacional Los Glaciares (Estancia Cristina, Puerto Bandera, Estancia Anita) (Imberti, 2005); Meseta Lago Strobel (Márquez et al., 2005); Estancia La Angostura (Imberti, unpubl. b).

Museum specimens. Lago Pueyrredón, 1 m, 10 Jun 1987, col. O. Padín, MLP.

Field observations. Bahía Redonda, Laguna Nimez, Parque Nacional Perito Moreno, Parque Nacional Los Glaciares, Laguna Azul, Río Gallegos, Estancia Rincón de los Morros, Estancia 9 de Julio, Puerto Bandera, Estancia La Soledad, Estancia Pali Aike, El Zurdo, Estancia Bella Vista Bitsch, Estancia Glencross, Estancia La Alice, Estancia Bella Vista (Imberti).

\section{Podilymbus podiceps antarcticus}

Literature. Parque Nacional Los Glaciares (El Chaltén, Puerto Bandera, Estancia Anita) (Imberti, 2005)

Field observations. Estancia 9 de Julio (Imberti).

\section{Podicephorus major major}

Literature. Puerto Deseado, Cañadón Misioneros (Oustalet, 1891); Puerto Deseado (Jardine \& Selby, 1830; Zapata, 1967; Nasca et al., 2004); Bahía Lángara (Zapata, 1969); Laguna Los Escarchados (Lange, 1981); Ría Deseado, Río Gallegos, Cabo Vírgenes (Yorio, 1998); Ría Deseado (Gandini \& Frere, 1998b); Laguna Nimez (Imberti \& Albrieu, 2001); Estancia La Angostura (Imberti, unpubl. b).

Museum specimens. Bahía del Fondo, $1 \mathrm{~m}, 27$ Dec 1921, col. A. Merkle, MLP; Caleta Olivia, $1 \mathrm{f}$, skeleton, 7 May 1974, col. M. Rumboll and E. Shaw; Puerto Deseado, 2 f, 11 Jan 1916, col. J. Mogensen; 1 ?, 4 Dec 1961, col. A. Zapata, MACN; Río Gallegos, 1 ?, 4 Dec 1914, col. S. Shipton, FML. Field observations. Ría Deseado, Río Gallegos, Ría Gallegos, Punta Loyola, Ría Coig, Monte León, Cabo Vírgenes (Imberti).

\section{Podicephorus major navasi}

Literature. Río Mayer (Scott \& Sharpe, 1904); Parque Nacional Los Glaciares (Imberti, 2005); Lago Argentino, Estancia Anita, Estancia La Soledad (Coconier, 2005).

Field observations. Parque Nacional Perito Moreno, Parque Nacional Los Glaciares, Puerto Bandera, Laguna Nimez, Río Gallegos, Río La Leona, Lago Buenos Aires, Lago San Martín (Imberti).

\section{Podiceps occipitalis occipitalis}

Literature. Laguna Los Escarchados, Laguna del Blanquillo, Laguna Escondida (Lange, 1981); Reserva de Vida Silvestre Los Escarchados (Erize, 1983); Laguna de la Nevada (Nuechterlein \& Storer, 1985); Estancia El Cuadro (De Lucca \& Saggese, 1992); Meseta del Tobiano, Laguna Los Escarchados, Meseta Lago Strobel, Lago Quiroga (Iglesias \& Pérez, 1998); Ría Deseado (Yorio, 1998); Laguna Nimez (Imberti \& Albrieu, 2001); Parque Nacional Los Glaciares (Puerto Bandera, Estancia Anita, Lago Argentino, Lago Roca) (Imberti, 2005); Meseta Lago Strobel, Río Chico, Estancia La Soledad, Estancia Anita (Coconier, 2005).

Museum specimens. Laguna del Blanquillo, 1 ?, 12 Feb 1980, col. J. Rodríguez Mata, MACN.

Field observations. Monumento Natural Bosques Petrificados, Parque Nacional Perito Moreno, Parque Nacional Los Glaciares, Ría Coig, Ría Deseado, Ría Gallegos, Estancia 9 de Julio, Estancia La Angostura, Monte León, Estancia El Cóndor, Estancia Cabo Buen Tiempo, Bahía San Julián, Laguna Los Escarchados (Imberti).

\section{Podiceps gallardoi}

Literature. Laguna Los Escarchados (Rumboll, 1974; Lange, 1981; Navas \& Camperi, 1999); La- 
guna Los Escarchados, Laguna Escondida, Laguna del Blanquillo (Lange, 1981); Reserva de Vida Silvestre Los Escarchados (Erize, 1983); Laguna de la Nevada (Straneck \& Johnson, 1984); Laguna de la Nevada, El Calafate (Nuechterlein \& Storer, 1985); Laguna Los Escarchados, Laguna del Blanquillo, Estancia El Tero, Laguna de la Nevada, Meseta del Tobiano, Meseta Lago Strobel (Fjeldså, 1986); Laguna Roble (Martín, 1992); lagoons located between $46^{\circ} \& 50^{\circ} \mathrm{S}$ and $70^{\circ} \& 72 \mathrm{c}$ W (Beltrán et al., 1992); estuary of Río Coig (Anónimo, 1994); Parque Nacional Perito Moreno (Chebez et al., 1998); Bañados del Tero and Bañados del Río Pelque, Meseta del Tobiano, Laguna Los Escarchados, Meseta Lago Strobel, Lago Quiroga, Laguna Roble (Iglesias \& Pérez, 1998); Ría Coig (Yorio, 1998); estuary of Río Gallegos (Imberti, 2003; Torres \& Vargas, 2005); Bahía San Julián, mouth of Río Santa Cruz, Paraje Le Marchand, estuary of Río Coig, estuary of Río Gallegos, Punta Bustamante, Baliza Población, Punta Loyola (Imberti et al., 2004); Río Gallegos, Río Coig, Meseta Lago Strobel, Laguna Tonchi, Meseta Lago Buenos Aires, Meseta del Asador (Coconier, 2005); Meseta Lago Strobel (Márquez et al., 2005); Río Gallegos, Río Coig, Meseta Lago Strobel, Río Chico, Parque Nacional Perito Moreno, Meseta Lago Buenos Aires, Meseta del Asador, Bahía San Julián (Di Giácomo et al., 2005); Estancia La Angostura (Imberti, unpubl. b).

Museum specimens. El Calafate $(80 \mathrm{~km}$ eastern), 1 f, 27 Apr 1974, col. M. Rumboll and E. Shaw, holotipus; Lago Viedma, Laguna Las Toscas, 3 ?, 27 Jan 1983, col. G. Nuechterlein and A. Johnson; Laguna del Blanquillo, $1 \mathrm{~m}, 19 \mathrm{Feb}$ 1981, col. G. Nuechterlein; Laguna Los Escarchados, 2 m, 16 May 1975, 8 Jan 1976, col. M. Rumboll, MACN.

Field observations. Lago Buenos Aires, Lago Cardiel Chico, Meseta Lago Strobel, Meseta del Asador, Estancia La Siberia, Laguna Tonchi, Laguna Roble, Ría Coig, Ría Gallegos, Estancia Cabo Buen Tiempo, Laguna Los Escarchados, Punta Loyola (Imberti); Parque Nacional Perito Moreno (M. Martínez pers. comm.)

\section{DIOMEDEIDAE}

\section{Thalassarche melanophris melanophris}

Literature. Golfo de San Jorge (Steullet \& Deautier, 1935); Ría Deseado (Gandini \& Frere, 1998b).

Field observations. Cabo Vírgenes, Punta Loyola, Estancia Cabo Buen Tiempo, Monte León, Ea. Cóndor, Puerto Coig, San Julián, Puerto Santa Cruz (Imberti).

\section{Diomedea exulans exulans}

Field observations. Cabo Vírgenes (Imberti).

\section{PROCELLARIIDAE}

\section{Macronectes giganteus}

Literature. Isla Leones, San Julián (Gould \& Darwin, 1838-1841); Bahía del Fondo (Steullet \& Deautier, 1935); Puerto Deseado (Zapata, 1967; Nasca et al., 2004); Ría Deseado (Gandini \& Frere, 1998b); Cabo Vírgenes, Río Deseado, Monte León, Río Gallegos, Río Coig (Coconier, 2005; Di Giácomo et al., 2005)

Field observations. Cabo Vírgenes, Punta Loyola, Ría Coig, Puerto Coig, San Julián, Puerto Santa Cruz, Ría Deseado, Monte León, Ea. Cóndor, Ría Gallegos (Imberti).

\section{Macronectes halli}

Literature. Cabo Vírgenes (Imberti, 2003).

Field observations. Cabo Vírgenes (Imberti).

\section{Fulmarus glacialoides}

Literature. Río Gallegos (Salvadori, 1900), Ría Deseado (Gandini \& Frere, 1998b).

Field observations. Cabo Vírgenes, Punta Loyola, Estancia Cabo Buen Tiempo, Estancia El Cóndor, Ría Coig (Imberti).

\section{Daption capense capense}

Literature. Río Gallegos (Salvador, 1900).

Field observations. Ría Gallegos (Pearman pers. comm.).

Procellaria aequinoctialis aequinoctialis Field observations. Cabo Vírgenes, Punta Loyola, Monte León, Estancia Cabo Buen Tiempo, Estancia El Cóndor, Ría Coig, Ría Gallegos (Imberti).

\section{Puffinus puffinus}

Literature. Cabo Vírgenes (Imberti, unpubl. a).

\section{Puffinus griseus}

Literature. Ría Deseado (Gandini \& Frere, 1998b).

Field observations. Cabo Vírgenes, Punta Loyola (Imberti).

\section{Puffinus gravis}

Field observations. Cabo Vírgenes, Punta Loyola (Imberti).

\section{HYDROBATIDAE}

Oceanites oceanicus ssp.

Literature. Río Gallegos (Salvadori, 1900) 


\section{PELECANOIDIDAE}

\section{Pelecanoides magellani}

Literature. Río Gallegos, between Río Gallegos and Santa Cruz, Cabo Vírgenes (Murphy \& Harper, 1921).

Field observations. Ría Coig, Cabo Vírgenes (Imberti).

\section{PHALACROCORACIDAE}

\section{Phalacrocorax gaimardi}

Literature. Puerto Deseado (Oustalet, 1891; Doello Jurado, 1917; Daneri, 1959; Zapata, 1967; Humphrey \& Bridge, 1970; Daciuk, 1977; de la Peña, 1980, 1987, 2005; Gandini \& Frere 1998b); Río Santa Cruz (Beck, 1912); Ría Deseado (Renard, 1931); Río Deseado, San Julián (Hellmayr \& Conover, 1948a); San Julián (Olrog, 1948); Cabo Blanco (Zapata, 1965, 1969; Gandini \& Frere, 1995; Nasca et al., 2004); Cabo Blanco, San Julián, Bahia San Jorge (Olrog, 1973); Puerto Santa Cruz, Puerto Deseado, San Julián (Jehl \& Rumboll, 1976); Ría Deseado (Albrieu \& Navarro, 1997; Millones et al., 2005); Monte Loayza, Cabo Blanco, Ría Deseado, Bahía Oso Marino, Bahía Laura, Bahía San Julián, Península San Julián, Ría Santa Cruz, Monte León (Yorio, 1998); Bahía Sanguinetto, Cabo Blanco, Río Deseado, Bahía Oso Marino (Gandini \& Frere, 1998a); Bahía San Julián, Monte León (Frere \& Gandini, 1998); Isla Elena, Cañadón del Puerto (Frere \& Gandini, 2001); Cabo Vírgenes, estuary of Río Gallegos (Imberti, 2003); Isla Elena (Gandini et al., 2005); Monte Loayza, Cabo Blanco, Isla Elena, Cañadón del Puerto, Isla Pingüino, Monte León (Frere et al., 2005); Río Deseado, Monte León (Coconier, 2005); Río Deseado, Monte León, Bahía San Julián (Di Giácomo et al., 2005).

Museum specimens. Puerto Deseado, 2 ?, 12 Jan 1916, col. S. Shipton, FML; 1 m, 10 Aug 1961, col. A. Zapata, MACN; 1 ?, 19 Jan 1972, C. Olrog, FML; $1 \mathrm{~m}$, skeleton, 10 Oct 1979, col. P.S. Humphrey, MACN.

Field observations. Cabo Blanco, Ría Deseado, Bahía San Julián, occasional Bahía Laura (Imberti), occasional Cabo Vírgenes (Albrieu pers. comm.).

\section{Phalacrocorax brasilianus brasilianus}

Literature. Isla Leones, Cañadón Misioneros (Oustalet, 1891); Isla Leones (Salvadori, 1900); Puerto Deseado (Doello Jurado, 1917; Hellmayr \& Conover, 1948a; Bó, 1956; Daneri, 1959; Zapata, 1967; Daciuk, 1977; de la Peña, 1987, 2005; Gandini \& Frere, 1998b; Nasca et al., 2004); Ma- zarredo (Zapata, 1969); Puerto Deseado, Río Gallegos (Humphrey \& Bridge, 1970); Río Turbio (Lucero, 1987); Ría Deseado (Albrieu \& Navarro, 1997); Monte Loayza, Ría Deseado, Punta Medanosa, Bahía Laura, Bahía San Julián, Península San Julián, Ría Santa Cruz, Cabo Vírgenes (Yorio, 1998); Río Deseado, Punta Buque (Gandini \& Frere, 1998a); Bahía San Julián (Frere \& Gandini, 1998); Laguna Nimez (Imberti \& Albrieu, 2001); Isla de los Pájaros, Isla Deseada (Frere et al., 2005); Parque Nacional Los Glaciares (Puerto Bandera, Lago Roca, Río Santa Cruz and Río La Leona) (Imberti, 2005); Río Deseado, Isla Deseada (Coconier, 2005); Estancia La Angostura (Imberti, unpubl. b).

Museum specimens. Puerto Deseado, $1 \mathrm{~m}$, skeleton, 26 Jan 1981, col. P.S. Humphrey, MACN.

Field observations. Ría Gallegos, Río Gallegos, Parque Nacional Perito Moreno, Parque Nacional Los Glaciares, Lago Posadas, Punta Loyola, Ría Deseado, Estancia Rincón de los Morros, Estancia Rupai Pacha, Ría Coig, Cabo Vírgenes, Monte León, Estancia Cabo Buen Tiempo, Puente Blanco, Ría Santa Cruz, Comandante Luis Piedra Buena, Bahía San Julián, Estancia Bella Vista (Imberti).

\section{Phalacrocorax magellanicus}

Literature. Río Santa Cruz (Carrara, 1952); Puerto Deseado (Zapata, 1967; Humphrey \& Bridge, 1970; de la Peña, 1980,1987, 2005; Gandini \& Frere, 1995; Gandini \& Frere, 1998b; Nasca et al., 2004); Cabo Tres Puntas (Zapata, 1969); Ría Deseado (Renard, 1931; Albrieu \& Navarro, 1997); Monte Loayza, Cabo Blanco, Ría Deseado, Bahía Oso Marino, Bahía Laura, Bahía San Julián, Península San Julián, Monte León, Cabo Vírgenes (Yorio, 1998); Punta de los Pájaros, Bahía Sanguinetto, Cabo Blanco, Río Deseado, Bahía Oso Marino, Isla Lobos (Gandini \& Frere, 1998a); Bahía Laura, Cerro Amette, Bahía San Julián, San Francisco de Paula, Pico Quebrado, Monte León, Cerro Observatorio, Faro Coig, Cabo Vírgenes (Frere \& Gandini, 1998); Punta de los Pájaros, Monte Loayza, Cabo Blanco, Isla Elena, Isla Pingüino, Isla Chata, Isla Lobos, Cerro Amette, Isla de Monte León, Cerro Observatorio, Faro Coig, Cabo Vírgenes (Frere et al., 2005); Río Deseado (Coconier, 2005).

Museum specimens. Puerto Deseado, 2 ?, 14 Dec 1915, S. Shipton, FML; 1 f, undated, col. J. Mogensen; 2 and 1 ?, 10 Aug and 28 Nov 1961, col. A. Zapata, MACN.

Field observations. Cabo Vírgenes, Monte León, Punta Loyola, Bahía San Julián, Bahía Laura (Imberti). 

Phalacrocorax atriceps atriceps and $\boldsymbol{P} . \boldsymbol{a}$.
albiventer

Literature. Puerto San Julián (Gould \& Darwin, 1838-1841; Gray, 1844; Sharpe \& Ogilvie-Grant, 1898); Cañadón Misioneros, Río Gallegos (Oustalet, 1891); Isla Leones (Salvadori, 1900); Ría Coig, Cabo Buen Tiempo, Monte Tigre (Scott \& Sharpe, 1915); Ría Coig (Murphy, 1916); Puerto Deseado (Doello Jurado, 1917; Hellmayr \& Conover, 1948; Daneri, 1959; Zapata, 1967; de la Peña, 1987, 2005; Gandini \& Frere, 1998b); Ría Deseado (Renard, 1931); Caleta Olivia, Cabo Blanco (Zapata, 1969); Río Gallegos, Puerto Deseado (Jehl \& Rumboll, 1976); Cabo Vírgenes (Goodall et al., 1991); Ría Deseado (Albrieu \& Navarro, 1997); Isla Deseada (Arrighi \& Navarro, 1998; Ferrari et al., 2004); Lago Argentino (Iglesias \& Pérez, 1998); Monte Loayza, Cabo Blanco, Ría Deseado, Bahía Oso Marino, Punta Medanosa, Bahía Laura, Bahía and Península San Julián, Ría Santa Cruz, Monte León, Río Gallegos, Cabo Vírgenes (Yorio, 1998); Bahía Sanguinetto, Cabo Blanco, Bahía Oso Marino, Isla Guano, Punta Buque, Islote del Cabo (Gandini \& Frere, 1998a); Bahía Laura, Islote Chato, Bahía San Julián, Ría Santa Cruz, Pico Quebrado, Monte León, Isla Deseada (Frere \& Gandini, 1998); Puerto Deseado (Nasca et al., 2004); Monte Loayza, Cabo Blanco, Isla Guano, Islote Chato, Isla Leones, Isla de Monte León, Isla Deseada (Frere et al., 2005); Río Deseado, Monte León, Río Gallegos, Isla Deseada (Coconier, 2005).

Museum specimens. Puerto Deseado, 2 ?, 20 Dec 1915, S. Shipton, FML; 1 f, 5 Jan 1916, col. J. Mogensen; 1 f y 1 ?, skeleton, 10 Oct 1979, col. P.S. Humphrey, MACN; Río Gallegos, 2 ?, 15 and 21 Dec 1914, col. S. Shipton, FML.

Field observations. Cabo Vírgenes, Punta Loyola, Monte León, Ría Deseado, Ría Coig, Ría Gallegos, Puerto Coig, Estancia Cabo Buen Tiempo, Estancia El Cóndor, Ría Santa Cruz, Bahía San Julián (Imberti); Lago Posadas (Bernacchi pers. comm.).

\section{ARDEIDAE}

\section{Nycticorax nycticorax obscurus}

Literature. Río Chico, Río Santa Cruz, Lago Pueyrredón (Scott \& Sharpe, 1912); Lago Pueyrredón, Río Chico (Dabbene, 1913); Puerto Deseado (Doello Jurado, 1917; Zotta \& Da Fonseca, 1936; Zapata, 1967; Daciuk, 1977; Gandini \& Frere, 1995; Gandini \& Frere, 1998b); Ría Deseado (Renard, 1931); Monte Loayza, Ría Santa Cruz, Ría Coig, Río Gallegos, Cabo Vírgenes (Yorio, 1998); Río Deseado (Gandini \& Frere, 1998a); Bahía San Julián, Isla Deseada (Frere \&
Gandini, 1998); Los Antiguos (Earnshaw \& Earnshaw, 2000); Laguna Nimez (Imberti \& Albrieu, 2001); Parque Nacional Los Glaciares (Imberti, 2005); Isla Deseada, Estancia El Cóndor (Coconier, 2005); Estancia La Angostura (Imberti, unpubl. b).

Museum specimens. Las Heras, Estancia Roca Blanca, 1 ?, 10 Jan 1961, S. Pierotti, FML; Puerto Deseado, 1 f, 25 Jan 1916, col. J. Mogensen, MACN. Field observations. Bahía Redonda, Laguna Nimez, Parque Nacional Perito Moreno, Parque Nacional Los Glaciares, Ría Gallegos, Lago Posadas, Estancia 9 de Julio, Punta Loyola, Monte León, Estancia Rincón de los Morros, Ría Coig, Estancia La Alice, Bahía San Julián, Estancia Bella Vista (Imberti).

\section{Egretta thula thula}

Literature. Puerto Deseado (Zapata, 1967; Humphrey \& Bridge, 1970)

\section{Ardea cocoi}

Literature. Punta Medanosa, Perito Moreno (Chebez et al., 1988), Ría Deseado, Punta Medanosa (Yorio, 1998); Estancia La Carreta, Bahía San Julián, Parque Nacional Los Glaciares (Imberti, 2003), Parque Nacional Los Glaciares (Lago Argentino, Río Mitre) (Imberti, 2005); Estancia La Angostura (Imberti, unpubl. b).

Museum specimens. Lago Posadas, 1 ?, 20 Jul 1941, col. M. Birabén, MLP.

Field observations. Estancia La Carreta, Bahía San Julián, Río Chico, Brazo Rico (Sturzenbaum pers. comm.); Parque Nacional Perito Moreno; Bahía Redonda, Calafate (Imberti).

\section{Ardea alba egretta}

Literature. Lago Buenos Aires (Wetmore, 1926a); Puerto Deseado (Zapata, 1967)

Field observations. Estancia Rincón de los Morros (Imberti).

\section{Bubulcus ibis ibis}

Literature. Estancia El Cóndor, Río Gallegos (Rumboll \& Canevari, 1975); Estancia Anita, Laguna del Desierto, Laguna del Sello -Meseta Lago Buenos Aires-, Cabo Vírgenes, Cañadón Gapp, Cabo Blanco, Meseta Lago Strobel (Chebez et al., 1988); Estancia El Cuadro (De Lucca \& Saggese, 1992); Laguna Nimez (Imberti \& Albrieu, 2001); Parque Nacional Los Glaciares (Imberti, 2005); Estancia La Angostura (Imberti, unpubl. b).

Field observations. Monumento Natural Bosques Petrificados, Parque Nacional Perito Moreno, Parque Nacional Los Glaciares, La Esperanza, Ría Coig, Río Gallegos, Estancia La Alice, El Calafate (Imberti). 


\section{THRESKIORNITHIDAE}

\section{Plegadis chihi}

Literature. Estancia La Soledad, Estancia La Alice (Imberti, 2003), Puerto Bandera (Imberti, 2005)

Field observations. Estancia La Alice (Imberti); Estancia La Soledad (Bernacchi pers. comm.).

\section{Theristicus melanopis melanopis}

Literature. Isla Leones (Oustalet, 1891); Río Chico (Scott \& Sharpe, 1912); Río Gallegos (Hellmayr \& Conover, 1948a); Río Chico, Río Gallegos (Zapata \& Martínez, 1972); Laguna Los Escarchados (Lange, 1981); Reserva de Vida Silvestre Los Escarchados (Erize, 1983); Estancia La Carlota, Depto. Güer Aike, Cabo Vírgenes (Frere et al., 1992); Estancia El Cuadro (De Lucca \& Saggese, 1992); Río Gallegos, Cabo Vírgenes (Yorio, 1998); Isla Deseada, Cabo Vírgenes (Frere \& Gandini, 1998); Los Antiguos (Earnshaw \& Earnshaw, 2000); Laguna Nimez (Imberti \& Albrieu, 2001); Parque Nacional Los Glaciares (Cerro Moreno, El Chaltén) (Imberti, 2005); Isla Deseada, Estancia El Cóndor (Coconier, 2005).

Museum specimens. Río Gallegos, 2 ?, 3 Dec 1914, col. S. Shipton, FML.

Field observations. Monumento Natural Bosques Petrificados, Estancia Rincón de los Morros, Laguna Azul, Parque Nacional Perito Moreno, Parque Nacional Los Glaciares, Cabo Vírgenes, Punta Loyola, Lago Posadas, Estancia Rupai Pacha, Estancia 9 de Julio, Puerto Bandera, Estancia La Soledad, Estancia Pali Aike, Laguna Nimez, Estancia Sofía, Estancia Bella Vista Bitsch, Río Gallegos, Ría Gallegos, Estancia Cabo Buen Tiempo, Monte León, Estancia Glen Cross, Ría Coig, El Roda, Estancia La Angostura, Estancia María Aike, El Pluma, Estancia Bella Vista (Imberti).

\section{CATHARTIDAE}

\section{Coragyps atratus foetens}

Literature. Lago Roca (Babarskas \& Chebez, 1999)

\section{Cathartes aura jota}

Literature. El Calafate, Estancia Anita (Imberti, 2005)

Field observations. Monumento Natural Bosques Petrificados (Zancaner pers. comm.), Punta Loyola (Sturzenbaum pers. comm.).

\section{Vultur gryphus}

Literature. Puerto Deseado, Río Santa Cruz valley (Gould \& Darwin, 1838-1841); Río Deseado,
Río Gallegos, Cabo Vírgenes (Vinciguerra, 1884); Río Gallegos (Oustalet, 1891); Cabo Buen Tiempo (Scott \& Sharpe, 1915); Río Deseado (Steullet \& Deautier, 1936); Laguna del Blanquillo (Lange, 1981); Parque Nacional Los Glaciares (Glaciar Perito Moreno, Lago Argentino, El Chaltén, Lago Viedma, Cerro Rosado, Estancia La Querencia) (Imberti, 2005); Parque Nacional Los Glaciares (Coconier, 2005); Parque Nacional Perito Moreno, Laguna Nimez, Lago Argentino, Parque Nacional Los Glaciares, Estancia La Soledad, Estancia La Anita (Di Giácomo et al., 2005).

Museum specimens. Cabo Buen Tiempo, $1 \mathrm{~m}$, 16 Jul 1896, col. O. A. Peterson, USNM; Santa Cruz, 1 ?, skeleton, 24 Nov 1981, col. D.H. Ellis, MACN.

Field observations. Monumento Natural Bosques Petrificados, Río Oro canyon, Lago Posadas, Cerro Comisión de Elefantes, Lago San Martín, Parque Nacional Perito Moreno, Parque Nacional Los Glaciares, Estancia Verdadera Argentina, Estancia 9 de Julio, Puerto Bandera, Estancia La Soledad, Lago del Desierto, Estancia Rincón de los Morros, Estancia El Cóndor, Rospentek (Imberti).

\section{PHOENICOPTERIDAE}

\section{Phoenicopterus chilensis}

Literature. Manantial de la Leona (Burmeister, 1890); San Julián (Dabbene, 1920b); Ría Deseado (Renard, 1931); Puerto Deseado (Zapata, 1967); Laguna Los Escarchados (Lange, 1981); Reserva de Vida Silvestre Los Escarchados (Erize, 1983); Bañados del Tero and del Río Pelque (Scott \& Carbonell, 1986); Estancia El Cuadro, Monumento Natural Bosques Petrificados (De Lucca \& Saggese, 1992); Bañados del Tero, Bañados del Río Pelque Puerto Bandera (Iglesias \& Pérez, 1998); Monte Loayza, Punta Medanosa, Cabo Vírgenes (Yorio, 1998); Laguna Nimez (Imberti \& Albrieu, 2001); Parque Nacional Los Glaciares (Lago Roca, Brazo Rico) (Imberti, 2005); Río Gallegos, Río Coig, Meseta Lago Strobel, Río Chico, Meseta Lago Buenos Aires, El Zurdo, Estancia El Cóndor, Laguna Nimez, Meseta del Asador, Parque Nacional Los Glaciares (Coconier, 2005); Río Gallegos, Río Coig, Meseta Lago Strobel, Monumento Nacional Bosques Petrificados, Estancia El Cuadro, Parque Nacional Perito Moreno, Meseta Lago Buenos Aires, El Zurdo, Laguna Nimez, Lago Argentino, Meseta del Asador, Parque Nacional Los Glaciares, Estancia La Soledad, Estancia La Anita, Estancia El Cóndor, Bahía San Julián (Di Giácomo et al., 2005).

Museum specimens. Las Heras, Estancia Roca Blanca, 2 ?, 9 and 11 Jan 1961, col. O. Budin and J.C. Viera, FML. 
Field observations. Monumento Natural Bosques Petrificados, Laguna Nimez, Parque Nacional Perito Moreno, Parque Nacional Los Glaciares, Estancia Rincón de los Morros, Estancia Rupai Pacha, Laguna Cóndor, Estancia 9 de Julio, Laguna Los Escarchados, Puerto Bandera, Estancia La Soledad, El Zurdo, Cabo Vírgenes, Estancia Sofía, Estancia Bella Vista Bitsch, Estancia Morro Chico, Estancia Moy Aike Chico, Ría Coig, Estancia La Alice, El Roda, Estancia La Angostura, Estancia Bella Vista (Imberti).

\section{ANATIDAE}

\section{Coscoroba coscoroba}

Literature. Río Gallegos (Sclater \& Salvin, 1869; Cunningham, 1871); Río Chico (Scott \& Sharpe, 1912); Puerto Deseado (Zapata, 1967); Laguna Los Escarchados (Lange, 1981); Reserva de Vida Silvestre Los Escarchados (Erize, 1983), Estancia El Cuadro (De Lucca \& Saggese, 1992), Lagunas Escarchados, Meseta Lago Strobel, Lago Quiroga (Iglesias \& Pérez, 1998), Cabo Blanco, Ría Deseado (Yorio, 1998); Laguna Nimez (Imberti \& Albrieu, 2001); Parque Nacional Los Glaciares (Lago Roca, Puerto Bandera, Estancia Anita) (Imberti, 2005); Meseta Lago Strobel, Río Chico, Bahía Redonda, Lago Argentino, Estancia Anita, Estancia La Soledad, Estancia El cóndor (Coconier, 2005); Cabo Vírgenes (Imberti, unpubl. a)

Field observations. Monumento Natural Bosques Petrificados, Río Gallegos, Bahía Redonda, Laguna Nimez, Parque Nacional Perito Moreno, Parque Nacional Los Glaciares, Lago Posadas, Estancia Rupai Pacha, Laguna Cóndor, Estancia La Angostura, Laguna Azul, Estancia Rincón de los Morros, Ría Coig, Puente Blanco, Comandante Luis Piedra Buena, Bahía San Julián, El Roda, Estancia Bella Vista (Imberti).

\section{Cygnus melanocorypha}

Literature. Río Coig (Scott \& Sharpe, 1912); Río Gallegos (Steullet \& Deautier, 1936), Bahía Lángara (Zapata, 1969); Laguna Los Escarchados (Lange, 1981); Reserva de Vida Silvestre Los Escarchados (Erize, 1983); Bañados del Tero, Bañados del Río Pelque (Scott \& Carbonell, 1986); Estancia El Cuadro (De Lucca \& Saggese, 1992); Bañados del Tero, Bañados del Río Pelque, Lago Argentino, Puerto Bandera, Lago Viedma (Iglesias \& Pérez, 1998); Ría Santa Cruz, Monte León (Yorio, 1998); Laguna Nimez (Imberti \& Albrieu, 2001); Parque Nacional Los Glaciares (Lago Roca, Puerto Bandera, Estancia Anita), Lago Argentino (Imberti, 2005); Meseta Lago Strobel, Río Chico, Bahía Redonda, Lago Argentino, Estancia El
Cóndor, Estancia Anita, Estancia La Soledad (Coconier, 2005); Meseta Lago Strobel (Márquez et al., 2005); Cabo Vírgenes (Imberti, unpubl. a) Museum specimens. Las Heras, Estancia Roca Blanca, 1 ?, 13 Jan 1961, col. O. Budin and J.C. Viera Puerto Deseado, 8 ?, 25 Jan 1916, col. S. Shipton, FML; Río Gallegos, 1 f, Nov 1897, collector unknown, MLP.

Field observations. Monumento Natural Bosques Petrificados, Río Gallegos, Bahía Redonda, Laguna Nimez, Lago Posadas, Lago Pueyrredón, Laguna Cóndor, Estancia 9 de Julio, Estancia El Tero, Estancia Rupai Pacha, Parque Nacional Perito Moreno, Parque Nacional Los Glaciares, Estancia Rincón de los Morros, Puerto Bandera, Estancia La Soledad, Puerto Coig, Estancia La Angostura, Estancia La Alice, Comandante Luis Piedra Buena, El Roda, Laguna Los Escarchados, Estancia Bella Vista (Imberti).

\section{Chloephaga picta picta}

Literature. Ría Coig, Río Coig (Scott \& Sharpe, 1912); Chonque Aike, Emelk-aik (Burmeister, 1890); Lago Argentino (Hellmayr, 1932; Hellmayr \& Conover, 1948a); Río Santa Cruz, Lago Argentino, Santa Cruz, Comandante Luis Piedra Buena, San Julián, Río Gallegos (Pergolani de Costa, 1955); Perito Moreno, Lago Belgrano, Cabo Blanco, Gobernador Gregores, San Julián, northern Río Coig (Plotnick, 1961); Puerto Deseado (Zapata, 1967; Daciuk, 1977), Cabo Blanco (Zapata, 1969); Río Gallegos (Olrog, 1975); Río Gallegos, Comandante Luis Piedra Buena, Puerto Deseado (Olrog, 1976); Laguna Los Escarchados (Lange, 1981); Río Gallegos, Puerto Deseado, Los Manantiales, Tapi Aike, Gobernador Gregores, Lago Little Hill, Laguna Cóndor, Las Heras, Lago Belgrano, Lago Posadas, Estancia El Milagro -Bajo Caracoles-, Río Belgrano -Perito Moreno-, Parque Nacional Perito Moreno, Estancia Cañadón del Chara, Río Rubens (Lucero, 1982); Reserva de Vida Silvestre Los Escarchados (Erize, 1983); Bañados del Tero, Bañados del Río Pelque (Scott \& Carbonell, 1986); Río Turbio, La Esperanza (de la Peña, 1987); Lago Burmeister, Río Robles (Laredo, 1988); Estancia El Cuadro, Monumento Natural Bosques Petrificados (De Lucca \& Saggese, 1992); Bañados del Tero, Bañados del Río Pelque, Lago Argentino, Puerto Bandera, Lago Viedma, Meseta del Tobiano, Laguna Los Escarchados, Meseta Lago Strobel, Lago Quiroga (Iglesias \& Pérez, 1998); Cabo Vírgenes (Yorio, 1998), Estancia El Cóndor, Bahía Posesión, Depto. Güer Aike (Cadierno \& Amorós, 1999); Laguna Nimez (Imberti \& Albrieu, 2001); Río Turbio (de la Peña, 2005); Parque Nacional Los Glaciares (Lago Roca) (Imberti, 2005); Estancia 
La Soledad, Estancia Anita, Estancia El Cóndor (Coconier, 2005); Río Gallegos, Río Coig, Meseta Lago Strobel, Río Chico, Monumento Natural Bosques Petrificados, Estancia El Cuadro, Parque Nacional Perito Moreno, Meseta Lago Buenos Aires, El Zurdo, Laguna Nimez, Lago Argentino, Meseta del Asador, Parque Nacional Los Glaciares, Estancia La Soledad, Estancia La Anita, Estancia El Cóndor, Bahía San Julián (Di Giácomo et al., 2005).

Museum specimens. Estancia La Renania, $1 \mathrm{~m}$, 24 Apr 1949; Lago Argentino, 1 m, Mar 1936, col. M. Birabén; Lago Belgrano, 1 f, Mar 1936, col. M. Birabén, MLP; Las Heras, 3 ?, 9 and 13 Jan 1961, col. O. Budin and J.C. Viera, FML; Río Gallegos, 2 ?, 18 Nov 1914, S. Shipton, FML; $1 \mathrm{~m}$ and $1 \mathrm{f}, 4$ Dec 1914, col. J. Mogensen; 1 m, 1 Jun 1972, col. A. Kovacs, MACN.

Field observations. Monumento Natural Bosques Petrificados, Bahía Redonda, Laguna Nimez, Parque Nacional Perito Moreno, Parque Nacional Los Glaciares, Lago Posadas, Río Gallegos, Estancia Rincón de los Morros, Estancia Rupai Pacha, Cabo Vírgenes, Estancia 9 de Julio, Laguna Los Escarchados, Puerto Bandera, Estancia La Soledad, Estancia Pali Aike, El Zurdo, Estancia La Angostura, Ría Coig, Río Coig, Laguna Azul, Punta Loyola, Estancia Sofía, Estancia Bella Vista Bitsch, Estancia Cabo Buen Tiempo, Estancia Moy Aike Chico, Estancia San Lorenzo, Estancia El Cóndor, Estancia Cóndor, Estancia Glencross, Estancia La Alice, Estancia Morro Chico, Ría Santa Cruz, El Roda, El Pluma, Estancia Bella Vista (Imberti).

\section{Chloephaga hybrida hybrida}

Field observations. Cabo Vírgenes, Estancia Rincón de los Morros (Imberti).

\section{Chloephaga poliocephala}

Literature. Lago Argentino (Hellmayr \& Conover, 1948a); Laguna Los Escarchados (Lange, 1981); Lago Little Hill, Río Turbio (Lucero, 1982); Río Turbio (de la Peña, 1987, 2005); Lago Argentino, Puerto Bandera, Lago Viedma, Meseta del Tobiano, Laguna Los Escarchados, Meseta Lago Strobel, Lago Quiroga (Iglesias \& Pérez, 1998); Laguna Nimez (Imberti \& Albrieu, 2001); Parque Nacional Los Glaciares (Imberti, 2005); Estancia La Soledad, Estancia Anita, Estancia El Cóndor (Coconier, 2005); Cabo Vírgenes, Monte León, Río Chico, Parque Nacional Perito Moreno, Laguna Nimez, Lago Argentino, Parque Nacional Los Glaciares, Estancia La Soledad, Estancia La Anita, Estancia El Cóndor (Di Giácomo et al., 2005); Cabo Vírgenes (Imberti, unpubl. a); Estancia La Angostura (Imberti, unpubl. b).
Museum specimens. Estancia Arbilla, $1 \mathrm{~m}$, skeleton, 30 Oct 2003, col. R.E. Wilson, MACN.

Field observations. Bahía Redonda, Laguna Nimez, Parque Nacional Perito Moreno, Parque Nacional Los Glaciares, Lago Posadas, Río Gallegos, Estancia Rincón de los Morros, Estancia Rupai Pacha, Estancia 9 de Julio, Puerto Bandera, Estancia La Soledad, El Zurdo, Estancia La Maipú, Estancia Glencross, El Roda (Imberti).

\section{Chloephaga rubidiceps}

Literature. Estancia El Cóndor (Rumboll, 1975; Cadierno \& Amorós, 1999); Cabo Vírgenes (Imberti, 2003); Cabo Vírgenes, Río Gallegos, El Zurdo, Estancia El Cóndor (Coconier, 2005); Cabo Vírgenes, Río Gallegos, Río Coig, Monumento Natural Bosques Petrificados, Estancia El Cuadro, El Zurdo, Estancia El Cóndor (Di Giácomo et al., 2005).

Museum specimens. Lago Argentino, 1 ?, Feb 1927, col. E. Budin, FML.

Field observations. Estancia La Angostura, Estancia Rincón de los Morros, Estancia Rupai Pacha, Río Gallegos, Ría Gallegos, Cabo Vírgenes, Estancia El Cóndor (Imberti).

\section{Merganetta armata armata}

Literature. Parque Nacional Los Glaciares, Río Frías (Imberti, 2005)

Field observations. Parque Nacional Perito Moreno, Parque Nacional Los Glaciares, Río de las Vueltas, Río La Leona (Imberti).

\section{Lophonetta specularioides specularioides}

Literature. Vicinity of Santa Cruz (Oustalet, 1891); Monte Tigre (Scott \& Sharpe, 1912); Ría Deseado (Renard, 1931); Río Gallegos (Hellmayr, 1932); Río Gallegos, Puerto Deseado (Hellmayr \& Conover, 1948a); Puerto Deseado (Zapata, 1967; Daciuk, 1977; Gandini \& Frere, 1998b; de la Peña, 2005); Punta Maqueda (Zapata, 1969); Reserva de Vida Silvestre Los Escarchados (Erize, 1983); Estancia El Cuadro, Monumento Natural Bosques Petrificados (De Lucca \& Saggese, 1992); Bañados del Tero, Bañados del Río Pelque (Scott \& Carbonell, 1986; Iglesias \& Pérez, 1998); Monte Loayza, Cabo Blanco, Ría Deseado, Bahía Laura, Río Gallegos, Cabo Vírgenes (Yorio, 1998); Río Deseado (Gandini \& Frere, 1998a); Isla Deseada (Frere \& Gandini, 1998); Laguna Nimez (Imberti \& Albrieu, 2001); Parque Nacional Los Glaciares (Imberti, 2005); Isla Deseada, Meseta Lago Strobel, Río Chico, Bahía Redonda, Lago Argentino, Estancia La Soledad, Estancia Anita, Estancia El Cóndor (Coconier, 2005); Meseta Lago Strobel (Márquez et al., 2005).

Museum specimens. Bajo Caracoles, Route 40, 1 f, skeleton, 28 Oct 2003, col. K. McCracken; 
Estancia Monte Dinero, $1 \mathrm{~m}$, skeleton, 5 Nov 2003, col. R.E. Wilson; Lago Argentino, Punta Bandera, $1 \mathrm{~m}, 22$ Feb 1959, col. P. Miles; Lago Viedma, Estancia Santa Margarita, $1 \mathrm{f}$, skeleton, 31 Oct 2003, col. K. McCracken; Paraje Le Marchand, 1 f, skeleton, 6 Nov 2003, col. R.E. Wilson; Puerto Deseado, 1 m, 3 Jan 1916, col. J. Mogensen; 2 f, 1 and 22 Aug 1961, col. A. Zapata, MACN; 2 ?, 13 Jan 1972, col. C. Olrog, FML.

Field observations. Monumento Natural Bosques Petrificados, Bahía Redonda, Estancia Rupai Pacha, Laguna Nimez, Estancia Rincón de los Morros, Cabo Vírgenes, Parque Nacional Perito Moreno, Parque Nacional Los Glaciares, Río Gallegos, Ría Gallegos, Ría Coig, Punta Loyola, Estancia Sofía, Estancia Cabo Buen Tiempo, Monte León, Estancia La Alice, Estancia Morro Chico, Ría Santa Cruz, Bahía San Julián, El Roda, Estancia La Angostura, Laguna Los Escarchados, Estancia Bella Vista (Imberti).

\section{Tachyeres patachonicus}

Literature. Río Gallegos, Puerto Deseado (Hellmayr \& Conover, 1948a); Puerto Deseado (Zapata, 1967; Daciuk, 1977; Humphrey \& Livezey, 1982; Livezey \& Humphrey, 1983; Gandini \& Frere, 1998b; de la Peña, 2005); Mazarredo (Zapata, 1969); Laguna Los Escarchados (Lange, 1981); Reserva de Vida Silvestre Los Escarchados (Erize, 1983); Laguna de la Nevada, El Calafate (Nuechterlein \& Storer, 1985); Estancia El Cuadro (De Lucca \& Saggese, 1992); Meseta del Tobiano, Laguna Los Escarchados, Meseta Lago Strobel, Lago Quiroga (Iglesias \& Pérez, 1998), Monte Loayza, Cabo Blanco, Ría Deseado (Yorio, 1998); Río Deseado (Gandini \& Frere, 1998a); Bahía San Julián (Frere \& Gandini, 1998); Laguna Nimez (Imberti \& Albrieu, 2001); Parque Nacional Los Glaciares (Imberti, 2005); Río Coig, Meseta Lago Strobel, Río Chico, Bahía Redonda, Lago Argentino, Estancia La Soledad, Estancia Anita, Estancia El Cóndor (Coconier, 2005); Río Coig (Di Giácomo et al., 2005); Cabo Vírgenes (Imberti, unpubl. a); Estancia La Angostura (Imberti, unpubl. b).

Museum specimens. Estancia La Angostura, 1 m, skeleton, 29 Oct 2003, col. R.E. Wilson; Lago Argentino, Puerto Bandera, 1 ?, skull, 22 Feb 1977, col. M. Rumboll; Laguna del Pescado, $1 \mathrm{~m}$, skeleton, 30 Oct 2003, col. R.E. Wilson, MACN; Puerto De-seado, 2 ?, 19 and 20 Jan 1972, col. C. Olrog, FML; 1 f, skeleton, 10 Oct 1979, col. P.S. Humphrey, MACN; Río Gallegos, 5 ?, 2 and 4 Dec 1914, col. S. Shipton, FML; Río Santa Cruz, Route $3,1 \mathrm{~m}$ and 1 ?, skeleton, 3 May 1974, col. M. Rumboll and E. Shaw, MACN.
Field observations. Monumento Natural Bosque Petrificados, Parque Nacional Perito Moreno, Parque Nacional Los Glaciares, Ría Coig, Monte León, Ría Deseado, Estancia Rupai Pacha, Río Gallegos, Estancia Rincón de los Morros, Puerto Bandera, Estancia La Soledad, Laguna Nimez, Monte León, Estancia Cabo Buen Tiempo, Ría Santa Cruz, Bahía San Julián, Laguna Azul (Imberti).

\section{Tachyeres pteneres}

Literature. Puerto Deseado (de la Peña, 1987)

Museum specimens. Estancia La Angostura, 1 m, skeleton, 9 Nov 2003, col. R.E. Wilson, MACN.

\section{Tachyeres leucocephalus}

Literature. Bahía San Julián, estuario del Río Deseado, Bahía Laura (Imberti, 2003)

Field observations. Puerto Deseado, Bahía Laura (Imberti); San Julián (Pearman pers. comm.).

\section{Speculanas specularis}

Literature. Río Mitre, Bella Vista, (Casares, 1935); Bella Vista (Hellmayr \& Conover, 1948a); Laguna Nimez (Imberti \& Albrieu, 2001); Parque Nacional Los Glaciares (Bahía del Túnel, Río de las Vueltas) (Imberti, 2005); Parque Nacional Los Glaciares (Coconier, 2005); Parque Nacional Perito Moreno, Parque Nacional Los Glaciares, Estancia La Soledad, Estancia La Anita (Di Giácomo et al., 2005).

Field observations. Estancia La Angostura, Parque Nacional Perito Moreno, Parque Nacional Los Glaciares, Estancia Rincón de los Morros, Río de las Vueltas, Estancia Achalay (Imberti).

\section{Anas platalea}

Literature. Río Coig (Scott \& Sharpe, 1912); Laguna Los Escarchados (Lange, 1981); Reserva de Vida Silvestre Los Escarchados (Erize, 1983); Laguna de la Nevada, El Calafate (Nuechterlein \& Storer, 1985); Bañados del Tero, Bañados del Río Pelque (Scott \& Carbonell, 1986); Estancia El Cuadro (De Lucca \& Saggese, 1992); Bañados del Tero, Bañados del Río Pelque, Meseta del Tobiano, Lagunas Escarchados, Meseta Lago Strobel, Lago Quiroga (Iglesias \& Pérez, 1998); Laguna Nimez (Imberti \& Albrieu, 2001); Parque Nacional Los Glaciares (Imberti, 2005); Meseta Lago Strobel, Río Chico, Bahía Redonda, Lago Argentino, Estancia La Soledad, Estancia Anita (Coconier, 2005); Meseta Lago Strobel (Márquez et al., 2005); Cabo Vírgenes (Imberti, unpubl. a). Field observations. Monumento Natural Bosques Petrificados, Bahía Redonda, Laguna 
Nimez, Estancia 9 de Julio, Parque Nacional Perito Moreno, Parque Nacional Los Glaciares, Río Gallegos, Estancia Rincón de los Morros, Estancia Rupai Pacha, Estancia La Angostura, Puerto Bandera, Estancia La Soledad, El Zurdo, Estancia La Alice, El Roda, Estancia María Aike (Imberti).

\section{Anas discors}

Literature. Laguna Los Escarchados (Rumboll, 1991).

\section{Anas cyanoptera cyanoptera}

Literature. Estancia El Cuadro (De Lucca \& Saggese, 1992), Puerto Bandera (Iglesias \& Pérez, 1998); Parque Nacional Los Glaciares (Puerto Bandera, Río de las Vueltas), Laguna Nimez (Imberti, 2005); Cabo Vírgenes (Imberti, unpubl. a). Museum specimens. Estancia La Angostura, 1 m, skeleton, 29 Oct 2003, col. R.E. Wilson, MACN. Field observations. Monumento Natural Bosques Petrificados, Punta Bandera, Estancia La Angostura (Imberti).

\section{Anas versicolor fretensis}

Literature. Río Ecker, Río Chico (Scott \& Sharpe, 1912); Estancia El Cuadro (De Lucca \& Saggese, 1992); Laguna Nimez (Imberti \& Albrieu, 2001); Parque Nacional Los Glaciares (Bahía del Túnel) (Imberti, 2005);

Museum specimens. Estancia La Angostura, 1 $\mathrm{m}$ and $1 \mathrm{f}$, skeleton, 28 Oct 2003, col. R.E. Wilson, MACN. Santa Cruz, 1 ?, 13 Feb 1910, col. S. Shipton, FML.

Field observations. Monumento Natural Bosques Petrificados, Parque Nacional Los Glaciares, Parque Nacional Perito Moreno, Estancia Rincón de los Morros, Estancia Rupai Pacha, Estancia Pali Aike, El Zurdo, Laguna Nimez, Estancia La Angostura, Laguna Balton, Estancia Bella Vista (Imberti).

\section{Anas sibilatrix}

Literature. Río Gallegos (Oustalet, 1891); Río Coig (Scott \& Sharpe, 1912); Laguna Los Escarchados, Laguna del Blanquillo (Lange, 1981); Reserva de Vida Silvestre Los Escarchados (Erize, 1983); La Esperanza (de la Peña, 1987); Estancia El Cuadro (De Lucca \& Saggese, 1992), Meseta del Tobiano, Laguna Los Escarchados, Meseta Lago Strobel, Lago Quiroga (Iglesias \& Pérez, 1998); Los Antiguos (Earnshaw \& Earnshaw, 2000); Laguna Nimez (Imberti \& Albrieu, 2001); Parque Nacional Los Glaciares (Bahía Túnel, Lago Roca, Puerto Bandera (Imberti, 2005); Meseta Lago Strobel, Río Chico,
Bahía Redonda, Lago Argentino, Estancia La Soledad, Estancia Anita, Estancia El Cóndor (Coconier, 2005); Meseta Lago Strobel (Márquez et al., 2005).

Museum specimens. Estancia El Cóndor, $1 \mathrm{~m}$, skeleton, 5 Nov 2003, col. K. McCracken; Gobernador Gregores, 1 f, 28 Oct 2003, col. R.E. Wilson; Punta Loyola, $1 \mathrm{~m}$ and $1 \mathrm{f}$, skeleton, 5 Nov 2003, col. K. McCracken, MACN; Río Gallegos, 2 ?, 20 Nov 1914, col. S. Shipton, FML; 1 f, 3 Dec 1914, col. J. Mogensen, MACN.

Field observations. Monumento Natural Bosques Petrificados, Bahía Redonda, Laguna Nimez, Parque Nacional Perito Moreno, Parque Nacional Los Glaciares, Estancia Rupai Pacha, Lago Posadas, Río Gallegos, Ría Gallegos, Estancia Rincón de los Morros, Estancia 9 de Julio, Cabo Vírgenes, Puerto Bandera, Estancia La Soledad, El Zurdo, Estancia La Angostura, Estancia Sofía, Estancia Bella Vista Bitsch, Estancia San Lorenzo, Laguna Los Escarchados, Estancia Bella Vista (Imberti).

\section{Anas flavirostris flavirostris}

Literature. Santa Cruz (Oustalet, 1891); Monte Tigre, Río Coig (Scott \& Sharpe, 1912); Puerto Deseado (Zapata, 1967); Laguna Los Escarchados (Lange, 1981); Reserva de Vida Silvestre Los Escarchados (Erize, 1983); Laguna Nimez (Imberti \& Albrieu, 2001); Estancia El Cuadro (De Lucca \& Saggese, 1992); Parque Nacional Los Glaciares (Imberti, 2005); Estancia El Cóndor (Coconier, 2005); Meseta Lago Strobel (Márquez et al., 2005).

Museum specimens. Coti Aike, $1 \mathrm{~m}$, skeleton, 1 Nov 2003, col. K. McCracken; Estancia La Frontera, $1 \mathrm{~m}$, skeleton, 26 Oct 2003; Estancia Las Tres Marías, Ruta 40, 1 f, skeleton, 1 Nov 2003, col. R.E. Wilson, MACN; Las Heras, Estancia Roca Blanca, 3 ?, 7 and 10 Jan 1961, col. O. Budin and J.C. Viera, FML.

Field observations. Monumento Nacional Bosques Petrificados, Río Gallegos, Ría Gallegos, Punta Loyola, Bahía Redonda, Laguna Nimez, Parque Nacional Perito Moreno, Parque Nacional Los Glaciares, Estancia Rincón de los Morros, Estancia Rupai Pacha, Estancia 9 de Julio, Cabo Vírgenes, Puerto Bandera, El Zurdo, Estancia La Soledad, Lago del Desierto, Estancia San Lorenzo, Estancia Sofía, Monte León, Estancia La Angostura, Estancia María Aike, Laguna Los Escarchados, Estancia Bella Vista (Imberti).

\section{Anas bahamensis rubrirostris}

Literature. Laguna Nimez (Imberti \& Albrieu, 2001); Puerto Bandera, Laguna Nimez (Imberti, 2005). 
Field observations. Laguna Nimez, Estancia La Soledad (Bernacchi and Roil pers. comm.).

\section{Anas georgica spinicauda}

Literature. Río Santa Cruz (Oustalet, 1891); Monte Tigre (Scott \& Sharpe, 1912); Laguna Los Escarchados (Lange, 1981); Reserva de Vida Silvestre Los Escarchados (Erize, 1983); Laguna de la Nevada, El Calafate (Nuechterlein \& Storer, 1985); Estancia El Cuadro (De Lucca \& Saggese, 1992); Meseta del Tobiano, Laguna Los Escarchados, Meseta Lago Strobel, Lago Quiroga (Iglesias \& Pérez, 1998); Laguna Nimez (Imberti \& Albrieu, 2001); Parque Nacional Los Glaciares (Imberti, 2005); Estancia El Cóndor (Coconier, 2005); Cabo Vírgenes (Imberti, unpubl. a).

Museum specimens. Estancia La Angostura, 1 $\mathrm{m}$, skeleton, 29 Oct 2003; Estancia Stag River, $1 \mathrm{f}$, skeleton, 3 Nov 2003; Gobernador Gregores, Río Chico, 1 m, skeleton, 28 Oct 2003; Los Antiguos, $1 \mathrm{~m}$, skeleton, 25 Oct 2003, col. K. McCracken; Coti Aike, 1 m, skeleton, 1 Nov 2003, col. R.E. Wilson, MACN.

Field observations. Monumento Natural Bosques Petrificados, Parque Nacional Perito Moreno, Parque Nacional Los Glaciares, Punta Loyola, Río Gallegos, Ría Gallegos, Estancia Rincón de los Morros, Cabo Vírgenes, Estancia Rupai Pacha, Estancia 9 de Julio, Laguna Los Escarchados, Laguna Nimez, El Zurdo, Estancia Pali Aike, Estancia La Angostura, Laguna Azul, Estancia Bella Vista Bitsch, El Roda, Estancia María Aike, Estancia Bella Vista (Imberti).

\section{Netta peposaca}

Literature. Laguna Los Escarchados (Lange, 1981; Fjeldså, 1985); Meseta de las Vizcachas, Punta Bandera, Estancia Alta Vista, Estancia San Lorenzo, Meseta La Siberia, Estancia Los Gurises, Ría San Julián (Chebez et al., 1988); Parque Nacional Los Glaciares (Lago Roca), Sierra Baguales, Lago Argentino, Laguna Nimez (Imberti, 2005); Estancia El Cóndor (Coconier, 2005); Estancia La Angostura (Imberti, unpubl. b).

Museum specimens. Estancia Stag River, Route 40, 2 f, skeleton, 3 Nov 2003, col. R.E. Wilson, MACN.

Field observations. Monumento Natural Bosques Petrificados, Estancia Rupai Pacha (Sturzenbaum com. pers.).

\section{Heteronetta atricapilla}

Literature. Lago Argentino (Coconier, 2005).

Field observations. Laguna Nimez (fide Pearman).

\section{Oxyura ferruginea}

Literature. Lago Argentino, Puerto Bandera, Lago Viedma (Iglesias \& Pérez, 1998); Laguna Nimez (Imberti \& Albrieu, 2001); Parque Nacional Los Glaciares (Imberti, 2005); Estancia La Soledad, Estancia Anita (Coconier, 2005); Meseta Lago Strobel (Márquez et al., 2005); Estancia La Angostura (Imberti, unpubl. b).

Museum specimens. Perito Moreno, Laguna Los Cisnes, 1 m, skeleton, 24 Oct 2003, col. R.E. Wilson, MACN.

Field observations. Parque Nacional Perito Moreno, Parque Nacional Los Glaciares, Punta Bandera, Estancia La Soledad, Estancia 9 de Julio, Estancia La Lucha (Imberti),

\section{Oxyura vittata}

Literature. Estancia El Cuadro (De Lucca \& Saggese, 1992); Lago Argentino, Puerto Bandera, Lago Viedma (Iglesias \& Pérez, 1998); Laguna Nimez (Imberti \& Albrieu, 2001); Parque Nacional Los Glaciares (Puerto Bandera, Estancia Cristina, Estancia Anita, Lago Roca, El Chaltén, Río de las Vueltas) (Imberti, 2005); Estancia La Soledad, Estancia Anita (Coconier, 2005).

Field observations. Monumento Natural Bosques Petrificados, Parque Nacional Los Glaciares, Punta Bandera, Estancia 9 de Julio, Estancia La Angostura (Imberti).

\section{ACCIPITRIDAE}

\section{Circus buffoni}

Literature. Río Gallegos (Olrog, 1948; Humphrey \& Bridge, 1970); Puerto Deseado (Zapata, 1967)

Field observations. Monumento Natural Bosques Petrificados (Zancaner pers. comm.).

\section{Circus cinereus}

Literature. Ría Coig, Río Chico (Scott \& Sharpe, 1915); Monte León (Hellmayr \& Conover, 1949); Estancia El Cuadro (De Lucca \& Saggese, 1992; De Lucca et al., 1993; Saggese \& De Lucca, 1995; Saggese \& De Lucca, 2001); Laguna Nimez (Imberti \& Albrieu, 2001); Parque Nacional Los Glaciares (Puerto Bandera) (Imberti, 2005); Río Gallegos, Río Coig, Río Chico, Monumento Nacional Bosques Petrificados, Estancia El Cuadro, Parque Nacional Perito Moreno, Meseta Lago Buenos Aires, El Zurdo, Laguna Nimez, Lago Argentino, Meseta del Asador, Estancia El Cóndor, Bahía San Julián (Di Giácomo et al., 2005).

Field observations. Monumento Natural Bosques Petrificados, Parque Nacional Perito Moreno, Parque Nacional Los Glaciares, Punta 
Bandera, Estancia Rincón de los Morros, Estancia 9 de Julio, Cabo Vírgenes, Lago Posadas, Estancia Rupai Pacha, Laguna Los Escarchados, Laguna Nimez, Estancia La Soledad, Estancia La Carreta, Ría Coig, Estancia Bella Vista Bitsch, Río Gallegos, Ría Gallegos, Estancia San Lorenzo, Estancia Cabo Buen Tiempo, Estancia La Angostura (Imberti).

\section{Accipiter bicolor chilensis}

Literature. Puerto Deseado (Zapata, 1967); Río Gallegos (Imberti, 2003); Parque Nacional Los Glaciares (Imberti, 2005).

Museum specimens. Lago Argentino, Cerro Mayo, 1 m, 18 Feb 1959, col. P. Miles, MACN.

Field observations. Parque Nacional Perito Moreno, Parque Nacional Los Glaciares, Estancia Cóndor (Imberti).

\section{Geranoaetus melanoleucus australis}

Literature. Santa Cruz (Oustalet, 1891); Estancia Los Pozos, Ría Coig (Scott \& Sharpe, 1915); Cerro Dorotea, Bahía del Fondo (Steullet \& Deautier, 1936); Fitz Roy (Zapata, 1969); Laguna Los Escarchados, Laguna del Blanquillo, Laguna Escondida (Lange, 1981); Estancia El Cuadro, Monumento Natural Bosques Petrificados (De Lucca \& Saggese, 1992); Estancia El Cuadro (De Lucca et al., 1993 De Lucca \& Saggese 1993; Saggese \& De Lucca, 1995; De Lucca \& Saggese, 1995; Saggese \& De Lucca, 2001); Laguna Nimez (Imberti \& Albrieu, 2001); Parque Nacional Los Glaciares (El Chaltén) (Imberti, 2005); Río Gallegos, Río Coig, Meseta Lago Strobel, Río Chico, Monumento Nacional Bosques Petrificados, Estancia El Cuadro, Parque Nacional Perito Moreno, Meseta Lago Buenos Aires, El Zurdo, Laguna Nimez, Lago Argentino, Meseta del Asador, Parque Nacional Los Glaciares, Estancia La Soledad, Estancia La Anita, Estancia El Cóndor, Bahía San Julián (Di Giácomo et al., 2005); Estancia La Angostura (Imberti, unpubl. b).

Museum specimens. Bahía del Fondo, 1 f, 31 Dec 1921, col. A. Merkle; Cerro Dorotea, 1 f, Apr 1898, collector unknown, MLP; Estancia El Tranquilo, Depto. Deseado, $2 \mathrm{~m}$ and $1 \mathrm{f}, 29$ and 30 Jan 1961; Estancia Roca Blanca, Depto. Magallanes, $2 \mathrm{~m}$ and 1 f, 20-26 Dec 1960, col. A. Budin and J. Viera, FML.

Field observations. Monumento Natural Bosques Petrificados, Estancia La Angostura, Parque Nacional Perito Moreno, Parque Nacional Los Glaciares, Lago Posadas, Estancia Cóndor, Lago Argentino, Estancia Rupai Pacha, Estancia 9 de Julio, Laguna Los Escarchados, Puerto Bandera, Estancia La Soledad, Estancia Anita, Cabo Vírgenes, Estancia Cabo Buen Tiempo,
Estancia La Alice, Punta Loyola, Estancia Palermo Aike, Estancia Rincón de los Morros, Estancia Bella Vista (Imberti).

\section{Buteo magnirostris pucherani}

Field observations. Monumento Natural Bosques Petrificados (Zoratti pers. comm.).

\section{Buteo polyosoma polyosoma}

Literature. Río Chico, Río Ecker, Ría Coig (Scott \& Sharpe, 1915); Lago Viedma (Steullet \& Deautier, 1936); Puerto Deseado (Zapata, 1967), Estancia El Cuadro, Monumento Natural Bosques Petrificados (De Lucca \& Saggese, 1992), Estancia El Cuadro (Saggese \& De Lucca, 2001); Parque Nacional Los Glaciares (Imberti, 2005); Cabo Vírgenes (Imberti, unpubl. a).

Museum specimens. Lago Viedma, 1 f, 3 Mar 1936, col. M. Birabén, MLP.

Field observations. Monumento Natural Bosques Petrificados, Parque Nacional Perito Moreno, Parque Nacional Los Glaciares, Lago Posadas, Estancia Cerro Ventana, Estancia La Angostura, Meseta Lago Strobel, Monte León, Lago San Martín, Lago Buenos Aires, El Calafate (Imberti).

\section{Buteo albigula}

Literature. Parque Nacional Perito Moreno (Chebez et al., 1998); Parque Nacional Los Glaciares (Seno Mayo) (Imberti, 2005).

Field observations. Parque Nacional Perito Moreno, Parque Nacional Los Glaciares (Zoratti pers. comm.).

\section{Buteo ventralis}

Literature. Santa Cruz $50^{\circ} \mathrm{S}$ (Gould \& Darwin, 1838-1841); Río Mitre, Parque Nacional Los Glaciares (El Chaltén) (Imberti, 2003); Parque Nacional Los Glaciares (Glaciar Perito Moreno and Lago Argentino) (Imberti, 2005).

Field observations. Parque Nacional Los Glaciares - Río Blanco, El Chaltén, Río Mitre (Imberti)

\section{FALCONIDAE}

\section{Phalcoboenus albogularis}

Literature. Santa Cruz (Gould, 1837a; Gould \& Darwin, 1838-1841; Hellmayr \& Conover, 1949); Río Chico (Burmeister, 1890); Caprek-aik (= Caprek Aike) (Burmeister, 1888); Río Gallegos (Oustalet, 1891); Río Gallegos, Río Ecker (Stone, 1915); Río Ecker, Río Gallegos, Río Santa Cruz, Río Chico, Cabo Buen Tiempo, Río Deseado (Scott \& Sharpe, 1915); Río Deseado (Steullet \& Deautier, 1936); Río Ecker (Hellmayr \& Conover, 1949); 
Parque Nacional Los Glaciares (Imberti, 2005); Parque Nacional Perito Moreno, Meseta Lago Buenos Aires, Meseta del Asador, Parque Nacional Los Glaciares, Estancia La Soledad, Estancia La Anita (Di Giácomo et al., 2005).

Museum specimens. Río Deseado, $1 \mathrm{f}$ and 1? Jun 1896, col. G. Gerling, MACN; 1 m, Mar 1897, col. G. Gerling, MLP.

Field observations. Parque Nacional Perito Moreno, Parque Nacional Los Glaciares, Meseta Lago Strobel, Estancia Rupai Pacha (Imberti).

\section{Caracara plancus plancus}

Literature. Isla Pavón (Oustalet, 1891); Río Gallegos (Scott \& Sharpe, 1915); Puerto Deseado (Zapata, 1967); Laguna Los Escarchados, Laguna Escondida (Lange, 1981); Reserva de Vida Silvestre Los Escarchados (Erize, 1983), Estancia El Cuadro (De Lucca \& Saggese, 1992; Saggese \& De Lucca, 2001); Laguna Nimez (Imberti \& Albrieu, 2001); Parque Nacional Los Glaciares (Imberti, 2005), Cabo Vírgenes (Imberti, unpubl. a).

Museum specimens. Estancia El Tranquilo, Depto. Deseado, 1 m, 31 Jan 1961, col. A. Budin and J. Viera, FML.

Field observations. Monumento Natural Bosques Petrificados, Parque Nacional Perito Moreno, Parque Nacional Los Glaciares, Puerto Bandera, Estancia Rincón de los Morros, Río Gallegos, Ría Gallegos, Lago Argentino, Estancia Rupai Pacha, Cabo Vírgenes, Estancia 9 de Julio, Laguna Los Escarchados, Estancia La Soledad, Estancia Pali Aike, El Zurdo, Estancia Bella Vista Bitsch, Ría Coig, Río Coig, Laguna Nimez, Punta Loyola, Estancia Sofía, Estancia Cóndor, Estancia Cabo Buen Tiempo, Estancia La Alice, Monte León, Estancia Moy Aike Chico, Estancia Glencross, Estancia El Cóndor, Ría Santa Cruz, Bahía San Julián, El Roda, Estancia La Angostura, Estancia María Aike, Estancia Bella Vista (Imberti).

\section{Milvago chimango temucoensis}

Literature. Puerto Deseado (Gould \& Darwin, 1838-1841); Laguna Los Escarchados (Lange, 1981); Laguna Nimez (Imberti \& Albrieu, 2001); Parque Nacional Los Glaciares (Imberti, 2005), Cabo Vírgenes (Imberti, unpubl. a).

Field observations. Parque Nacional Perito Moreno, Parque Nacional Los Glaciares, Puerto Bandera, Estancia Rincón de los Morros, Lago Posadas, Río Gallegos, Ría Gallegos, Estancia Rupai Pacha, Estancia 9 de Julio, Estancia La Soledad, Estancia Cabo Buen Tiempo, Estancia La Alice, Laguna Nimez, Ría Santa Cruz, Estancia La Angostura, Estancia María Aike (Imberti).

\section{Spiziapteryx circumcinctus}

Literature. Parque Nacional Los Glaciares (Lago Argentino) (Bornschein, 1996).

\section{Falco sparverius cinnamominus}

Literature. Río Chico (Burmeister, 1890; Cory 1915); Río Ecker, Río Chico (Scott \& Sharpe, 1915); Puerto Deseado (Zapata, 1967), Punta Maqueda (Zapata, 1969), Estancia El Cuadro (De Lucca, 1992, 1993 a, b; De Lucca et al., 1993; Saggese \& De Lucca, 2001); Estancia El Cuadro, Monumento Natural Bosques Petrificados (De Lucca \& Saggese, 1992); Los Antiguos (Earnshaw \& Earnshaw, 2000); Laguna Nimez (Imberti \& Albrieu, 2001); Parque Nacional Los Glaciares (Imberti, 2005), Cabo Vírgenes (Imberti, unpubl. a).

Museum specimens. Lago Argentino, Lago Frío, $1 \mathrm{~m}$ ?, 23 Jan 1959, col. P. Miles, MACN.

Field observations. Monumento Natural Bosques Petrificados, Parque Nacional Perito Moreno, Parque Nacional Los Glaciares, Estancia Rincón de los Morros, Estancia 9 de Julio, El Zurdo, Laguna Los Escarchados, Estancia La Angostura, Estancia Cóndor, Ría Coig, Punta Loyola, Río Gallegos, Ría Gallegos, Estancia El Cóndor, Estancia Morro Chico, Bahía San Julián, Estancia Glencross, El Pluma, Estancia Bella Vista (Imberti).

\section{Falco femoralis femoralis}

Literature. Puerto Deseado (Gould \& Darwin, 1838-1841; Martens, 1900; Hellmayr \& Conover, 1949; Zapata, 1967); Río Chico, Ría Coig (Scott \& Sharpe, 1915); Laguna Nimez (Imberti \& Albrieu, 2001); Parque Nacional Los Glaciares (Lago Roca, Glaciar Perito Moreno) (Imberti, 2005) Cabo Vírgenes (Imberti, unpubl. a).

Field observations. Parque Nacional Perito Moreno, Parque Nacional Los Glaciares, Estancia Rincón de los Morros, Rupai Pacha, Estancia La Angostura, Monte León, Ría Coig, Estancia Cabo Buen Tiempo, Estancia La Carlota (Imberti).

\section{Falco peregrinus cassini}

Literature. Puerto Deseado (Gurney, 1882); Ría Coig (Scott \& Sharpe, 1915); Laguna Los Escarchados (Lange, 1981); Estancia El Cuadro (De Lucca \& Saggese, 1992; Saggese \& De Lucca, 2001); Los Antiguos (Earnshaw \& Earnshaw, 2000); Laguna Nimez (Imberti \& Albrieu, 2001); Parque Nacional Los Glaciares (Glaciar Perito Moreno) (Imberti, 2005); Estancia La Angostura (Imberti, unpubl. b).

Museum specimens. Laguna Isola Bella, $1 \mathrm{~m}$, 15 Mar 1997, col. W.G. Vasina; Estancia Pali Aike, 1 f, 27 Nov 1988, col. W.G. Vasina, MACN; Puerto 
Deseado, $1 \mathrm{f}$ and 2 ?, 12-19 Jan 1916, col. S. Shipton, FML; Río Pinturas, 1 m, Nov 1981, col. W.G. Vasina, MACN.

Field observations. Monumento Natural Bosques Petrificados, Parque Nacional Perito Moreno, Parque Nacional Los Glaciares, Puerto Bandera, Ría Gallegos, Cabo Vírgenes, Meseta Lago Strobel, Estancia La Soledad, Laguna Azul, Monte León, Ría Coig, Estancia Glencross, El Zurdo, Ría Santa Cruz, Estancia Bella Vista (Imberti).

\section{RALLIDAE}

\section{Rallus antarcticus}

Literature. Río Chico inferior (Scott \& Sharpe, 1904); Estancia La Angostura, Río Chico (Mazar Barnett et al., 1998); Estancia La Estrella, Río Chico, El Zurdo (Pugnali et al., 2004); Estancia Soledad, Puerto Bandera, Estancia Anita, Estancia La Querencia, Laguna Nimez, Lago Viedma (Imberti, 2005); Río Chico, El Zurdo, Laguna Nimez, Parque Nacional Los Glaciares (Coconier, 2005); Río Chico, El Zurdo, Laguna Nimez, Lago Argentino, Parque Nacional Los Glaciares, Estancia La Soledad, Estancia La Anita (Di Giácomo et al., 2005).

Field observations. Estancia La Angostura, El Zurdo, Estancia Bella Vista Bitsch, Estancia La Soledad, Estancia Cerro Ventana, Laguna Nimez (Imberti); Estancia La Estrella (Pearman pers. comm.).

\section{Pardirallus sanguinolentus landbecki}

Literature. Caracoles (= Bajo Caracoles) (Wetmore, 1926a); Lago Argentino (Navas, 1991); Laguna Nimez (Imberti \& Albrieu, 2001); Parque Nacional Los Glaciares (Puerto Bandera) (Imberti, 2005); Estancia La Angostura (Imberti, unpubl. b).

Museum specimens. Lago Argentino, Punta Bandera, 1 f, 21 Feb 1959, col. P. Miles, MACN.

Field observations. Parque Nacional Los Glaciares, Punta Bandera, Estancia Cóndor, Estancia Pali Aike, Estancia La Soledad, Estancia La Alice, El Zurdo (Imberti).

\section{Porzana spiloptera}

Field observations. Caleta Olivia (Imberti).

\section{Fulica leucoptera}

Literature. Río Coig, Estancia Pali Aike, Río Ecker, Río Chico (Scott \& Sharpe, 1904); Puerto Deseado (Zapata, 1967); Laguna Los Escarchados (Lange, 1981); Reserva de Vida Silvestre Los Escarchados (Erize, 1983), Estancia El Cuadro (De Lucca \& Saggese, 1992); Laguna Nimez (Imberti
\& Albrieu, 2001); Parque Nacional Los Glaciares (Imberti, 2005).

Field observations. Monumento Natural Bosques Petrificados, Parque Nacional Perito Moreno, Parque Nacional Los Glaciares, Puerto Bandera, Estancia Rincón de los Morros, Estancia Rupai Pacha, Bahía Redonda, Laguna Nimez, Estancia 9 de Julio, Lago Posadas, Río Gallegos, Cabo Vírgenes, Estancia La Soledad, Estancia Pali Aike, Estancia La Angostura, Laguna Azul, Estancia Sofía, El Zurdo, Estancia San Lorenzo, Estancia Cóndor, Estancia Bella Vista (Imberti).

\section{Fulica armillata}

Literature. Río Chico, Río Deseado (Burmeister, 1890); Río Gallegos (Oustalet, 1891); Río Chico, Río Santa Cruz, Río Ecker, Río Coig, Río Deseado (Scott \& Sharpe, 1904); Laguna Los Escarchados (Lange, 1981); Reserva de Vida Silvestre Los Escarchados (Erize, 1983), Laguna de la Nevada, El Calafate (Nuechterlein \& Storer, 1985); Lago Argentino, Puerto Bandera, Lago Viedma, Meseta del Tobiano, Laguna Los Escarchados, Meseta Lago Strobel, Lago Quiroga (Iglesias \& Pérez, 1998); Laguna Nimez (Imberti \& Albrieu, 2001); Parque Nacional Los Glaciares (Imberti, 2005).

Field observations. Monumento Natural Bosques Petrificados, Parque Nacional Perito Moreno, Parque Nacional Los Glaciares, Estancia Rincón de los Morros, Estancia Bella Vista Bitsch, El Zurdo, Estancia La Alice, Laguna Azul, Estancia La Angostura (Imberti).

\section{Fulica rufifrons}

Literature. Parque Nacional Los Glaciares (Lago Roca, Puerto Bandera) (Imberti, 2005).

Museum specimens. Estancia Roca Blanca, 250 $\mathrm{km}$ south of Colonia Las Heras, Depto. Magallanes, 3 f, 29 Dec 1960, 7 Jan 1961, col. A. Budin and J. Viera, FML.

Field observations. El Zurdo, Estancia La Nélida (Imberti).

\section{HAEMATOPODIDAE}

\section{Haematopus palliatus palliatus}

Literature. Bahía del Fondo (Steullet \& Deautier, 1939); Puerto Deseado (Hellmayr \& Conover, 1948b); Caleta Olivia (Zapata, 1969); Caleta Olivia, Puerto Deseado, Bahía Laura, San Julián (Jehl \& Rumboll, 1976); San Julián (Jehl, 1978); Monte Loayza, Cabo Blanco, Punta Medanosa, Bahía Laura, Ría Santa Cruz (Yorio, 1998); Ría Deseado (Gandini \& Frere, 1998b); Estancia La Angostura (Imberti, unpubl. b).

Museum specimens. Bahía del Fondo, $1 \mathrm{~m}$ and 1 f, 16 Dec 1921, col. A. Merkle, MLP; Puerto 
Deseado, $2 \mathrm{~m}$ and $1 \mathrm{f}, 10$ and 27 Dec 1915, col. S. Shipton; 1 ?, 10 Jan 1972, col. C. Olrog, FML.

Field observations. Ría Gallegos, Ría Deseado, Ría Coig, Punta Loyola, Cabo Vírgenes, Ría Santa Cruz, Estancia Cabo Buen Tiempo, Bahía San Julián (Imberti).

\section{Haematopus ater}

Literature. Puerto Deseado (Oustalet, 1891; Zapata, 1967; Daciuk, 1977; de la Peña, 1987, 2005; Gandini \& Frere, 1998b); San Julián (Jehl, 1978); Monte Loayza, Cabo Blanco, Ría Deseado, Bahía Laura, Ría Santa Cruz (Yorio, 1998); Cabo Blanco, Río Deseado, Punta Buque (Gandini \& Frere, 1998a); Bahía San Julián, Ría Santa Cruz (Frere \& Gandini, 1998); Parque Nacional Los Glaciares (Glaciar Perito Moreno) (Imberti, 2005). Museum specimens. Puerto Deseado, $1 \mathrm{~m}$ and 1 f, 11 Dec 1915, col. S. Shipton, FML; 1 f, 20 Jul 1969, col. G. A. Voss, USNM.

Field observations. Parque Nacional Los Glaciares, Ría Deseado, Monte León, Ría Coig, Bahía San Julián (Imberti).

\section{Haematopus leucopodus}

Literature. Cañadón Misioneros (Oustalet, 1891); Mouth of Río Coig (Scott \& Sharpe, 1910); Río Gallegos (Murphy, 1925; Ferrari \& Albrieu, 2005); Puerto Deseado (Zapata, 1967; Daciuk, 1977); Punta Maqueda (Zapata, 1969); Estancia La Federica, Estancia Punta Alta (Humphrey \& Bridge, 1970); Estancia Güer Aike, Valle del Río Santa Cruz, Lago Argentino, Río Coig, Puerto Santa Cruz, El Salado, Bahía Laura (Jehl \& Rumboll, 1976); San Julián (Jehl, 1978); Río Gallegos -85 km NW- (Miller \& Baker, 1980); Laguna Los Escarchados (Lange, 1981); Reserva de Vida Silvestre Los Escarchados (Erize, 1983), Lago Viedma (Fjeldså, 1985); Cancha Carrera, La Esperanza (de la Peña, 1987, 2005); Río Bote, Meseta La Siberia, Río Guanaco -Parque Nacional Los Glaciares-, Meseta de las Vizcachas, Punta Bandera, Lago Roca -Estancia Alta Vista-, Laguna de los Escarchados (Chebez et al., 1988), Monte Loayza, Ría Deseado, Punta Medanosa, Ría Coig, Río Gallegos (Yorio, 1998); Ría Deseado (Gandini \& Frere, 1998b); Laguna Nimez (Imberti \& Albrieu, 2001); estuary of Río Gallegos (Imberti, 2003); Río Gallegos, Río Coig (Coconier, 2005).

Museum specimens. Puerto Deseado, 1 ?, 12 Jan 1972, col. C. Olrog; Río Gallegos, $1 \mathrm{~m}$ and 1 f, 22 Nov and 4 Dec 1914, col. S. Shipton, FML.

Field observations. Parque Nacional Perito Moreno, Parque Nacional Los Glaciares, Estancia Rincón de los Morros, Punta Loyola, Lago Posadas, Río Gallegos, Ría Gallegos, Estancia
Rupai Pacha, Cabo Vírgenes, Estancia Sofía, Monte León, Laguna Los Escarchados, Ría Coig, Ría Deseado, Estancia Cabo Buen Tiempo, Ría Santa Cruz, Bahía San Julián, El Roda (Imberti).

\section{RECURVIROSTRIDAE}

\section{Himantopus melanurus melanurus}

Literature. Laguna Nimez (Imberti \& Albrieu, 2001); El Calafate (Laguna Nimez), Estancia La Soledad (Imberti, 2003); Puerto Bandera (Imberti, 2005)

Field observations. Laguna Nimez, Estancia La Soledad (Bernacchi and Roil pers. comm.).

\section{CHARADRIIDAE}

\section{Vanellus chilensis fretensis}

Literature. Isla Leones (Oustalet, 1891); Río Coig, Río Gallegos (Scott \& Sharpe, 1910); Cheike, Río Chico (Brodkorb, 1934); Puerto Deseado (Zapata, 1967); Fitz Roy (Zapata, 1969); Laguna Los Escarchados (Lange, 1981); Reserva de Vida Silvestre Los Escarchados (Erize, 1983); Lago Roca, Puerto Deseado, Bahía Oso Marino (Navas \& Bó, 1986); Estancia El Cuadro (De Lucca \& Saggese, 1992; Saggese \& De Lucca, 2001); Cabo Vírgenes (Yorio, 1998); Laguna Nimez (Imberti \& Albrieu, 2001); Parque Nacional Los Glaciares (Lago Roca) (Imberti, 2005).

Museum specimens. Bahía del Fondo, 1 m, 14 Dec 1921, col. A. Merkle, MLP; Bahía Oso Marino, $1 \mathrm{f}, 25$ Dec 1985, col. P.S. Humphrey, MACN; Estancia Roca Blanca, $3 \mathrm{~m}$ and $1 \mathrm{f}, 29$ and 31 Dec 1960, 9 Jan 1961, col. A. Budin and J. Viera, FML; Lago Argentino, Lago Roca, 1 m, 16 Jan 1959, col. P. Miles, MACN; Río Gallegos, $1 \mathrm{~m}$ and 1 f, 25 Nov 1914, col. S. Shipton, FML.

Field observations. Monumento Natural Bosques Petrificados, Parque Nacional Perito Moreno, Parque Nacional Los Glaciares, Puerto Bandera, Estancia Rincón de los Morros, Estancia Rupai Pacha, Bahía Redonda, Estancia 9 de Julio, Río Gallegos, Ría Gallegos, Lago Posadas, Cabo Vírgenes, Estancia La Soledad, Estancia Pali Aike, Estancia La Angostura, Estancia Sofía, Estancia San Lorenzo, Estancia Bella Vista Bitsch, Laguna Nimez, Punta Loyola, Laguna Los Escarchados, Ría Coyle, Estancia Cóndor, Estancia San Lorenzo, Estancia Glencross, El Roda, Estancia María Aike, El Pluma (Imberti).

\section{Pluvialis dominica}

Literature. Punta Medanosa (Chebez et al., 1988; Yorio, 1998); Ría Deseado (Gandini \& Frere, 1998b). 


\section{Charadrius semipalmatus}

Literature. Puerto Deseado (Seebohm, 1887; Sharpe, 1896; Dabbene, 1920a).

\section{Charadrius falklandicus}

Literature. Puerto Deseado (Oustalet, 1891; Zapata, 1967; Daciuk, 1977); Puerto San Julián (Sharpe, 1896); Río Coig (Scott \& Sharpe, 1910); Mazarredo (Zapata, 1969); Laguna Los Escarchados (Lange, 1981); Reserva de Vida Silvestre Los Escarchados (Erize, 1983), Punta Medanosa, Bahía San Julián (Chebez et al., 1988), Estancia El Cuadro (De Lucca \& Saggese, 1992), Laguna Los Escarchados, Meseta Lago Strobel, Lago Quiroga (Iglesias \& Pérez, 1998), Cabo Blanco, Punta Medanosa, Río Gallegos, Cabo Vírgenes (Yorio, 1998); Punta Buque (Gandini \& Frere, 1998a); Ría Deseado (Gandini \& Frere, 1998b); Laguna Nimez (Imberti \& Albrieu, 2001); Parque Nacional Los Glaciares (Imberti, 2005); Río Gallegos, Río Coig, Bahía Redonda, Lago Argentino, Estancia El cóndor (Coconier, 2005); Río Gallegos (Ferrari \& Albrieu, 2005); Estancia La Angostura (Imberti, unpubl. b).

Museum specimens. Bahía del Fondo, $1 \mathrm{~m}, 30$ Dec 1921, col. A. Merkle, MLP; Estancia Roca Blanca, 2 f, 29 Dec 1960, col. A. Budin and J. Viera, FML; Puerto Deseado, 1 ?, 18 Oct 1961, col. A. Zapata; Río Gallegos, $1 \mathrm{~m}$ and $1 \mathrm{f}, 31$ Aug 1915, col. R. Beck, MACN, $1 \mathrm{~m}, 1 \mathrm{f}$ and 2 ?, 14 and 23 Nov, 4 Dec 1914, col. S. Shipton, FML.

Field observations. Monumento Natural Bosques Petrificados, Parque Nacional Perito Moreno, Estancia Rincón de los Morros, Estancia Rupai Pacha, Río Gallegos, Ría Gallegos, Cabo Vírgenes, Estancia Sofía, Estancia Bella Vista, Punta Loyola, Ría Coig, Meseta Lago Strobel, Laguna Los Escarchados, Estancia Morro Chico, El Roda, Bahía Laura (Imberti).

\section{Charadrius modestus}

Literature. Monte Tigre, Cabo Buen Tiempo, Río Ecker (Scott \& Sharpe, 1910); mouth of Río Gallegos (Wetmore, 1926a; Ferrari \& Albrieu, 2005); Laguna Los Escarchados (Lange, 1981); Reserva de Vida Silvestre Los Escarchados (Erize, 1983) Estancia El Cuadro (De Lucca \& Saggese, 1992) Laguna Los Escarchados, Meseta Lago Strobel, Lago Quiroga (Iglesias \& Pérez, 1998); Laguna Nimez (Imberti \& Albrieu, 2001); Laguna Travesía (Imberti, 2003); Parque Nacional Los Glaciares (Imberti, 2005); Río Gallegos, Estancia El Cóndor (Coconier, 2005); Río Gallegos, Río Coig, El Zurdo, Laguna Nimez, Lago Argentino, Parque Nacional Los Glaciares, Estancia La Soledad, Estancia La Anita, Estancia El Cóndor, Bahía San
Julián (Di Giácomo et al., 2005); Estancia La Angostura (Imberti, unpubl. b).

Field observations. Monumento Natural Bosques Petrificados, Parque Nacional Perito Moreno, Estancia Rincón de los Morros, Estancia Rupai Pacha, Laguna Nimez, Estancia 9 de Julio, Río Gallegos, Estancia Sofía, Estancia Buitreras, Estancia La Carlota, Estancia Morro Chico, Ría Coig, El Roda, Estancia Glencross (Imberti).

\section{Oreopholus ruficollis ruficollis}

Literature. Río Gallegos (Sclater et al., 1870; Hellmayr, 1932); Monte Tigre, Río Deseado, Río Chico (Scott \& Sharpe, 1910); Río Belgrano (Wetmore, 1926a); Paso Ibáñez, Río Gallegos (Hellmayr \& Conover, 1948b); Puerto Deseado (Zapata, 1967), Caleta Olivia (Zapata, 1969); Laguna Los Escarchados (Lange, 1981); Reserva de Vida Silvestre Los Escarchados (Erize, 1983), Estancia El Cuadro (De Lucca \& Saggese, 1992; Saggese \& De Lucca, 2001); Ría Deseado (Gandini \& Frere, 1998b); Parque Nacional Los Glaciares (Puerto Bandera) (Imberti, 2005), Meseta Lago Strobel (Coconier, 2005); Cabo Vírgenes (Imberti, unpubl. a)

Museum specimens. Estancia El Tranquilo, Depto. Deseado, $3 \mathrm{~m}$ and 1 ?, 10-20 Jan 1961; Estancia Roca Blanca, 2 m, 31 Dec 1960, 15 Jan 1961, col. A. Budin and J. Viera, FML; Puerto Deseado, 1 m, 16 Aug 1961, col. A. Zapata, MACN.

Field observations. Monumento Natural Bosques Petrificados, Parque Nacional Perito Moreno, Puerto Bandera, Estancia Rincón de los Morros, Estancia La Soledad, Estancia La Angostura, El Zurdo, Estancia El Cóndor, Estancia Bella Vista, Punta Loyola, Laguna Los Escarchados, Estancia Bella Vista Bitsch (Imberti).

\section{SCOLOPACIDAE}

\section{Gallinago paraguaiae magellanica}

Literature. Cañadón Misioneros (Oustalet, 1891); Río Mayer (Scott \& Sharpe, 1910); Laguna Los Escarchados (Lange, 1981); Reserva de Vida Silvestre Los Escarchados (Erize, 1983), Estancia El Cuadro (De Lucca \& Saggese, 1992); Los Antiguos (Earnshaw \& Earnshaw, 2000); Laguna Nimez (Imberti \& Albrieu, 2001); Parque Nacional Los Glaciares (Imberti, 2005); Bahía Redonda, Lago Argentino (Coconier, 2005); Estancia La Angostura (Imberti, unpubl. b).

Field observations. Monumento Natural Bosques Petrificados, Parque Nacional Perito Moreno, Parque Nacional Los Glaciares, Estancia Rincón de los Morros, Estancia Glencross, Laguna Nimez, Bahía Redonda, Cabo Vírgenes, Río 
Gallegos, Ría Gallegos, Estancia Rupai Pacha, Puerto Bandera, Estancia Bella Vista Bitsch, Estancia La Soledad, El Zurdo, Estancia Cóndor, Estancia La Alice, Estancia Bella Vista (Imberti).

\section{Gallinago stricklandii}

Literature. Cancha Carrera (Imberti, 2003); Parque Nacional Los Glaciares (Coconier, 2005). Field observations. Cancha Carrera (Imberti).

\section{Limosa haemastica}

Literature. Puerto Deseado (Sharpe, 1896, Dabbene, 1920a); Laguna Los Escarchados (Lange, 1981); Reserva de Vida Silvestre Los Escarchados (Erize, 1983); Punta Medanosa (Chebez et al., 1988); Punta Loyola (Bala et al., 1998); Cabo Blanco, Ría Deseado, Punta Medanosa, Bahía Laura (Yorio, 1998); Laguna Nimez (Imberti \& Albrieu, 2001); mouth of Río Gallegos (Imberti, 2003; Coconier, 2005; Ferrari \& Albrieu, 2005); Lago Argentino, Laguna Nimez, El Calafate (Imberti, 2005).

Museum specimens. Laguna Los Escarchados, $1 \mathrm{~m}, 4 \mathrm{Feb}$ 1976, col. M. Rumboll, MACN.

Field observations. Monumento Natural Bosques Petrificados, Estancia Rupai Pacha, Cabo Vírgenes, Ría Gallegos, Río Coig, Laguna Nimez (Imberti).

\section{Numenius phaeopus hudsonicus}

Literature. Punta Quilla - Puerto Santa Cruz (Chebez et al., 1988), Ría Deseado, Ría Santa Cruz (Yorio, 1998); Punta Quilla, Puerto Deseado, estuary of Río Gallegos, Bahía Laura, Cabo Vírgenes (Imberti, 2003); Parque Nacional Los Glaciares (Brazo Rico del Lago Argentino), Lago Argentino (Imberti, 2005); Río Gallegos (Coconier, 2005).

Field observations. Cabo Vírgenes, Ría Gallegos (Imberti).

\section{Tringa melanoleuca}

Literature. Caleta Olivia (Zapata, 1969); Laguna Nimez (Imberti \& Albrieu, 2001); Parque Nacional Los Glaciares (Lago Roca) (Imberti, 2005); Estancia La Angostura (Imberti, unpubl. b).

Field observations. Estancia La Angostura, Estancia Rincón de los Morros (Imberti); Parque Nacional Perito Moreno (M. Martínez pers. comm.)

\section{Tringa flavipes}

Literature. Caleta Olivia (Zapata, 1969); Laguna Nimez (Imberti \& Albrieu, 2001); Parque Nacional Los Glaciares (Lago Roca) (Imberti, 2005).

Field observations. Estancia La Angostura, Estancia Rincón de los Morros, Lago Posadas, Río Gallegos, Estancia Bella Vista Bitsch (Imberti).

\section{Arenaria interpres morinella}

Literature. Bahía de los Nodales (Jehl \& Rumboll, 1976)

\section{Calidris canutus rufa}

Literature. Punta Medanosa (Chebez et al., 1988), Cabo Blanco, Ría Deseado, Punta Medanosa (Yorio, 1998); Punta Buque (Gandini \& Frere, 1998a); Bahía San Julián (Hernández et al., 2004); Río Gallegos (Coconier, 2005; Ferrari \& Albrieu, 2005; Ferrari et al., 2005).

Field observations. Ría Gallegos, Punta Loyola (Imberti).

\section{Calidris alba}

Literature. Cabo Blanco, Río Gallegos, Cabo Vírgenes (Yorio, 1998); Punta Buque (Gandini \& Frere, 1998a).

Field observations. Ría Gallegos, Ría Coig, Punta Loyola (Imberti).

\section{Calidris fuscicollis}

Literature. Puerto San Julián (Sharpe, 1896); Estancia Pali Aike (Scott \& Sharpe, 1910); Puerto Deseado, Estancia Pali Aike, Río Gallegos, San Julián (Dabbene, 1920a); Puerto Deseado (Zapata, 1967); Río Gallegos (Steullet \& Deautier, 1939; Ferrari \& Albrieu, 2005); Mazarredo (Zapata, 1969); Laguna Los Escarchados (Lange, 1981); Reserva de Vida Silvestre Los Escarchados (Erize, 1983); Bañados del Tero, Bañados del Río Pelque (Scott \& Carbonell, 1986); Punta Medanosa, Bahía San Julián (Chebez et al., 1988); Bañados del Tero, Bañados del Río Pelque, Meseta del Tobiano, Laguna Los Escarchados, Meseta Lago Strobel, Lago Quiroga (Iglesias \& Pérez, 1998); Cabo Blanco, Punta Medanosa, Bahía Laura, Ría Coig (Yorio, 1998); Punta Buque (Gandini \& Frere, 1998a); Ría Deseado (Gandini \& Frere, 1998b); Laguna Nimez (Imberti \& Albrieu, 2001); Parque Nacional Los Glaciares (Imberti, 2005); Río Gallegos, Río Coig (Coconier, 2005); Estancia La Angostura (Imberti, unpubl. b).

Museum specimens. Laguna Los Escarchados, 1 m, 7 Jan 1976, col. M. Rumboll; Río Gallegos, 1 m, Feb 1897, MLP, 1 m, 27 Nov 1914, col. J. Mogensen, MACN, $1 \mathrm{~m}, 1 \mathrm{f}$ and 1 ?, 16 and $23 \mathrm{Nov}$ 1914, col. S. Shipton, FML.

Field observations. Ría Gallegos, Río Gallegos, Estancia Rincón de los Morros, Ría Coig, Monte León, Estancia Rupai Pacha, Cabo Vírgenes, Estancia Bella Vista Bitsch, Laguna Los Escarchados, Meseta Lago Strobel, Estancia Sofía, Estancia Morro Chico, El Roda, Bahía Laura, Estancia Bella Vista, Punta Loyola (Imberti). 


\section{Calidris bairdii}

Literature. Río Coig (Scott \& Sharpe, 1910; Dabbene, 1920a); Laguna Los Escarchados (Lange, 1981); Reserva de Vida Silvestre Los Escarchados (Erize, 1983); Meseta del Tobiano, Laguna Los Escarchados, Meseta Lago Strobel, Lago Quiroga (Iglesias \& Pérez, 1998); Cabo Blanco, Punta Medanosa (Yorio, 1998); Laguna Nimez (Imberti \& Albrieu, 2001); Parque Nacional Los Glaciares (Imberti, 2005); Río Gallegos, Bahía Redonda, Lago Argentino, Estancia El Cóndor (Coconier, 2005).

Museum specimens. Estancia Roca Blanca, 10 $\mathrm{m}$ and 2 f, 20 and 31 Dec 1960, 7-11 Jan 1961, col. A. Budin and J. Viera, FML.

Field observations. Monumento Natural Bosques Petrificados, Parque Nacional Perito Moreno, Parque Nacional Los Glaciares, Ría Gallegos, Río Gallegos, Estancia Rincón de los Morros, Ría Coig, Estancia Rupai Pacha, Cabo Vírgenes, Estancia Bella Vista Bitsch, Laguna Los Escarchados, Meseta Lago Strobel, Estancia Sofía, Estancia Morro Chico, Bahía Laura, Estancia Bella Vista, Estancia La Angostura, Punta Loyola (Imberti).

\section{Calidris melanotos}

Literature. Puerto Deseado (Sharpe, 1896; Dabbene, 1920a)

Field observations. Parque Nacional Perito Moreno, Estancia Sofía, Estancia Morro Chico, Punta Loyola (Imberti).

\section{Phalaropus tricolor}

Literature. Laguna Los Escarchados (Lange, 1981); Reserva de Vida Silvestre Los Escarchados (Erize, 1983), Meseta Lago Strobel, Lago Quiroga (Iglesias \& Pérez, 1998); Laguna Nimez (Imberti \& Albrieu, 2001); Parque Nacional Los Glaciares (Lago Roca, Puerto Bandera) (Imberti, 2005), Cabo Vírgenes (Imberti, unpubl. a); Estancia La Angostura (Imberti, unpubl. b).

Museum specimens. Tellier, 1 f, 13 Oct 1979, col. M.C. Thompson, MACN.

Field observations. Estancia Rincón de los Morros, Estancia Rupai Pacha, Meseta Lago Strobel, Estancia 9 de Julio (Imberti).

\section{Phalaropus fulicaria}

Literature. Laguna Nimez, Parque Nacional Los Glaciares (Bahía Viento Norte), Laguna Tonchi (Imberti, 2003); Lago Argentino (Imberti, 2005); Río Chico (Coconier, 2005); Estancia La Angostura (Imberti, unpubl. b).

Field observations. Laguna Nimez (Albrieu pers. comm.)

\section{THINOCORIDAE}

\section{Attagis gayi gayi}

Literature. Río Ecker (Scott \& Sharpe, 1910; Dabbene 1913); Cerro Colorado, southern Lago Buenos Aires (Wetmore, 1926a); Lago Buenos Aires (Hellmayr, 1932); Parque Nacional Los Glaciares (Imberti, 2005); Meseta Lago Strobel, Parque Nacional Perito Moreno, Meseta Lago Buenos Aires, Meseta del Asador, Parque Nacional Los Glaciares, Estancia La Soledad, Estancia La Anita (Di Giácomo et al., 2005); Estancia La Angostura (Imberti, unpubl. b).

Field observations. Parque Nacional Perito Moreno, Parque Nacional Los Glaciares, Estancia Verdadera Argentina (Imberti).

\section{Attagis malouinus}

Literature. Santa Cruz (Salvadori, 1900); Río Ecker, Estancia Killik Aike Norte (Scott \& Sharpe, 1910); Laguna Los Escarchados (Lange, 1981); Reserva de Vida Silvestre Los Escarchados (Erize, 1983); Estancia Cóndor, Laguna Los Escarchados (Navas \& Bó, 1996); Río Coig (Imberti, 2003); Parque Nacional Los Glaciares (Imberti, 2005); Estancia El Cóndor (Coconier, 2005); Río Gallegos, Río Coig, Meseta Lago Strobel, Parque Nacional Perito Moreno, Meseta Lago Buenos Aires, El Zurdo, Meseta del Asador, Estancia El Cóndor (Di Giácomo et al., 2005); Cabo Vírgenes (Imberti, unpubl. a); Estancia La Angostura (Imberti, unpubl. b).

Museum specimens. El Calafate, $1 \mathrm{f}, 11$ May 1975; Río Gallegos, 1 m, 2 May 1974, 2 f, 30 Apr 1974, $1 \mathrm{~m}, 1 \mathrm{f}$ and 1 ?, 11 May 1975, col. M. Rumboll, MACN.

Field observations. Parque Nacional Perito Moreno, Parque Nacional Los Glaciares, Estancia Cabo Buen Tiempo, Estancia Don Braulio, Estancia El Cóndor, Estancia La Carlota, Estancia Sofía, Estancia Coy Inlet, Laguna Los Escarchados, Estancia Bella Vista (Imberti).

\section{Thinocorus orbignyianus orbignyianus}

Literature. Río Chico (Scott \& Sharpe, 1910); Río Chico, Santa Cruz (Brodkorb, 1928); Lago Argentino (Hellmayr \& Conover, 1948b); Parque Nacional Los Glaciares (Imberti, 2005); Río Gallegos, Río Coig, Meseta Lago Strobel, Río Chico, Monumento Nacional Bosques Petrificados, Estancia El Cuadro, Parque Nacional Perito Moreno, Meseta Lago Buenos Aires, El Zurdo, Laguna Nimez, Lago Argentino, Meseta del Asador, Parque Nacional Los Glaciares, Estancia La Soledad, Estancia La Anita, Estancia El Cóndor (Di Giácomo et al., 2005). 
Museum specimens. Estancia Roca Blanca, 4 m, $4 \mathrm{f}$ and 1 ?, 27 and 30 Dec 1960, 15 Jan 1961; Pico Truncado, $50 \mathrm{~km}$ south of Colonia Las Heras, $1 \mathrm{~m}, 19$ Dec 1960, col. A. Budin and J. Viera, FML. Field observations. Parque Nacional Perito Moreno, Parque Nacional Los Glaciares, Monumento Natural Bosques Petrificados, Lago Posadas, Río Gallegos, Estancia Rupai Pacha, Estancia 9 de Julio, Punta Loyola, Meseta Lago Strobel, Estancia Las Tunas, Estancia Rincón de los Morros, Estancia La Angostura, Laguna Los Escarchados (Imberti).

\section{Thinocorus rumicivorus rumicivorus}

Literature. Laguna La Leona, vecinity of Santa Cruz, Cañadón Misioneros (Oustalet, 1891); Monte Tigre, Río Santa Cruz (Scott \& Sharpe, 1910); Río Santa Cruz (Reichenow, 1920); Ría Coig (Wetmore, 1926b); Bahía del Fondo, Lago Belgrano (Steullet \& Deautier, 1939); Paso Ibañez, Pampa Alta, Cerro Fortaleza, Río Gallegos, Río Santa Cruz, Monte Tigre (Hellmayr \& Conover, 1948b); Puerto Deseado (Zapata, 1967; Daciuk, 1977); Bahía Lángara, Fitz Roy, Mazarredo (Zapata, 1969); Laguna Los Escarchados (Lange, 1981); Reserva de Vida Silvestre Los Escarchados (Erize, 1983) Estancia El Cuadro (De Lucca \& Saggese, 1992) Laguna Los Escarchados, Meseta Lago Strobel, Lago Quiroga (Iglesias \& Pérez, 1998); Laguna Nimez (Imberti \& Albrieu, 2001); Puerto Deseado, Río Gallegos (de la Peña, 2005); Parque Nacional Los Glaciares (Imberti, 2005); Estancia El Cóndor (Coconier, 2005), Lago Argentino (Edelaar et al., 2005); Río Gallegos, Río Coig, Meseta Lago Strobel, Río Chico, Monumento Nacional Bosques Petrificados, Estancia El Cuadro, Parque Nacional Perito Moreno, Meseta Lago Buenos Aires, El Zurdo, Laguna Nimez, Lago Argentino, Meseta del Asador, Parque Nacional Los Glaciares, Estancia La Soledad, Estancia La Anita, Estancia El Cóndor, Bahía San Julián (Di Giácomo et al., 2005).

Museum specimens. Bahía del Fondo, $1 \mathrm{~m}$ and 1 f, 14 Dec 1921, col. A. Merkle, MLP; Estancia Roca Blanca, 1 f, 15 Jan 1961, col. A. Budin and J. Viera, FML; Lago Belgrano, 1 f, Mar 1936, col. M. Birabén, MLP; Perito Moreno, 1 ?, skeleton, Dec 1989; Río Gallegos, 1 ?, 22 Nov 1916, col. S. Shipton, FML; Tres Lagos, 1 m, skeleton, Jan 1991, col. L.M. Chiappe, MACN.

Field observations. Parque Nacional Perito Moreno, Parque Nacional Los Glaciares, Monumento Natural Bosques Petrificados, Lago Posadas, Río Gallegos, Ría Gallegos, Estancia Rupai Pacha, Estancia 9 de Julio, Estancia Rincón de los Morros, Estancia La Angostura, Cabo Vírgenes, Monte León, Estancia Bella Vista
Bitsch, Estancia Cabo Buen Tiempo, Ría Coig, Río Coig, Estancia El Cóndor, Estancia La Carlota, Estancia Sofía, El Roda, Estancia María Aike, Estancia Bella Vista, Laguna Los Escarchados (Imberti).

\section{PLUVIANELLIDAE}

\section{Pluvianellus socialis}

Literature. Río Coig (Scott \& Sharpe, 1910); Laguna Los Escarchados (Rumboll, 1974; Lange, 1981); Bahia de los Nodales, Estancia Tres de Enero (33 km S Río Gallegos), Paraje Le Marchand, Estancia Monte León, Comandante Luis Piedra Buena, San Julián, El Salado (Jehl, 1975); Laguna Los Escarchados, Tapi Aike (Jehl \& Rumboll, 1976); Reserva de Vida Silvestre Los Escarchados (Erize, 1983); Meseta Lago Strobel (Fjeldså, 1985) Bahía de San Julián, Laguna de los Escarchados, Meseta Lago Strobel, Meseta La Siberia, Lago Viedma, Laguna del Sello (Chebez et al., 1988) Estancia El Cuadro (De Lucca \& Saggese, 1992) Laguna Los Escarchados, Meseta Lago Strobel, Lago Quiroga, Laguna Roble (Iglesias \& Pérez, 1998), Bahía and Península San Julián (Yorio, 1998) Laguna Nimez (Imberti \& Albrieu, 2001); Estancia Rincón de Los Morros, Estancia Glencross, Río Leona (Paso Biggieri) (Imberti, 2003); Lago Argentino, Paso Biggieri, Estancia Glencross, Laguna Los Escarchados, Estancia La Angelina, Estuario Río Gallegos, Isla Deseada, Estuario Río Chico, Laguna Little Hill, Estuario Río Coig, Laguna Travesía, Estancia Rincón de los Morros (Ferrari et al,. 2003); Río Gallegos, Río Coig, Meseta Lago Strobel, Meseta Lago Buenos Aires, Laguna Nimez, Meseta del Asador (Coconier, 2005); Río Gallegos (Ferrari \& Albrieu, 2005); Río Gallegos, Río Coig, Meseta Lago Strobel, Parque Nacional Perito Moreno, Meseta Lago Buenos Aires, Laguna Nimez, Lago Argentino, Meseta del Asador, Bahía San Julián (Di Giácomo et al., 2005); Estancia La Angostura (Imberti, unpubl. b).

Field observations. Monumento Natural Bosques Petrificados, Parque Nacional Perito Moreno, Laguna Los Escarchados, Lago Cardiel, Ría Coig, El Roda, Estancia Rincón de los Morros, Estancia Glencross, Laguna Nimez, Lago Argentino, Laguna Morro Chico (Imberti).

\section{CHIONIDAE}

\section{Chionis alba}

Literature. Río Gallegos (Salvadori, 1900; Dabbene, 1910), Monte León (Steullet \& Deautier, 1939); from Río Gallegos to Puerto Deseado (Olrog, 1948); Puerto Deseado (Zapata, 1967), 
Monte Loayza, Río Gallegos, Cabo Vírgenes (Yorio, 1998); Ría Deseado (Gandini \& Frere, 1998b).

Museum specimens. Puerto Deseado, $1 \mathrm{f}, 22 \mathrm{Aug}$ 1961, col. A. Zapata, MACN, 1 m, 10 Jan 1972, col. C. Olrog, FML; $1 \mathrm{~m}$ and 2 f, 20 Jul 1969, col. G. A. Boss, USNM; Río Gallegos, $1 \mathrm{~m}$ and 1 f, 21 Dec 1914, col. S. Shipton, FML.

Field observations. Ría Gallegos, Punta Loyola, Ría Deseado, Monte León, Estancia Cabo Buen Tiempo, Bahía San Julián (Imberti).

\section{LARIDAE}

\section{Stercorarius chilensis}

Literature. Cañadón Misioneros (Oustalet, 1891); Isla Leones, Río Santa Cruz (Oates, 1901); Río Gallegos (Murphy, 1936); Puerto Deseado, Río Gallegos (Hellmayr \& Conover, 1948b); Puerto Deseado (Zapata, 1967; Gandini \& Frere, 1998b; Nasca et al., 2004); Ría Deseado, Bahía Oso Marino, Bahía Laura (Yorio, 1998); Río Deseado, Isla Deseada (Coconier, 2005).

Museum specimens. Puerto Deseado, $1 \mathrm{~m}, 12$ Jan 1916, col. J. Mogensen, MACN, 2 f, 20 Dec 1915, col. S. Shipton, 10 Jan 1972, col. C. Olrog; Río Gallegos, $2 \mathrm{~m}$ and 1f, 18 Feb and 19 Nov 1914, col. S. Shipton, FML.

Field observations. Ría Gallegos, Cabo Vírgenes, Monte León (Imberti).

\section{Stercorarius antarcticus antarcticus}

Literature. Bahía Oso Marino, Bahía Laura, Ría Santa Cruz, Río Gallegos, Cabo Vírgenes (Yorio, 1998), Puerto Deseado (Gandini \& Frere, 1998b; Nasca et al., 2004); Isla de los Pájaros, Isla Pingüino, Isla Leones (Yorio, 2005); Parque Nacional Los Glaciares (Glaciar Upsala, Glaciar Perito Moreno) (Imberti, 2005); Río Deseado (Coconier, 2005).

Museum specimens. Isla Pingüino, $11 \mathrm{~m}$ and 5 f, 8 and 9 Nov 1941, col. A. Umana, MLP.

Field observations. Ría Gallegos, Cabo Vírgenes, Punta Loyola, Ría Deseado, Estancia Cabo Buen Tiempo (Imberti).

\section{Stercorarius pomarinus}

Literature. Cabo Vírgenes (Imberti, unpubl. a).

\section{Stercorarius parasiticus}

Literature. Ría Deseado (Gandini \& Frere, 1998b).

Field observations. Punta Loyola (Howell and Webb pers. comm.).

\section{Chroicocephalus cirrocephalus}

Literature. Ría Coig, Cabo Buen Tiempo (Scott \& Sharpe, 1910); Puerto Deseado (Daneri, 1959)
Museum specimens. Río Gallegos, 1 f, 21 Dec 1914, col. S. Shipton, FML.

\section{Chroicocephalus maculipennis}

Literature. Ría Coig, Cabo Buen Tiempo (Scott \& Sharpe, 1910); Río Gallegos, Río Santa Cruz (Murphy, 1936); Puerto Deseado (Zapata, 1967); Laguna Los Escarchados (Lange, 1981); Ría Deseado, Río Gallegos, Cabo Vírgenes (Yorio, 1998); Ría Deseado (Gandini \& Frere, 1998b); Laguna Nimez (Imberti \& Albrieu, 2001); Parque Nacional Los Glaciares (Lago Roca) (Imberti, 2005); Estancia La Angostura (Imberti, unpubl. b).

Field observations. Parque Nacional Perito Moreno, Parque Nacional Los Glaciares, Estancia Rincón de los Morros, Estancia Rupai Pacha, Río Gallegos, Ría Gallegos, Ría Coig, Punta Loyola, Estancia Sofía, Estancia Cabo Buen Tiempo, Ría Santa Cruz, Bahía San Julián, Estancia Bella Vista, Cabo Vírgenes, Monte León (Imberti).

\section{Larus scoresbii}

Literature. Puerto San Julián (Gould \& Darwin, 1838-1841; Sclater \& Salvin 1871); Río Santa Cruz (Oustalet, 1891; Dabbene, 1910); Ría Deseado (Renard, 1931); Río Gallegos (Murphy, 1936); Bahía del Fondo (Steullet \& Deautier, 1939); Cabo Blanco (Zapata, 1965, 1969), Puerto Deseado (Zapata, 1967; Humphrey \& Bridge, 1970; Daciuk, 1977; Gandini \& Frere, 1998b); Monte Loayza, Cabo Blanco, Ría Deseado, Bahía Oso Marino, Punta Medanosa, Bahía San Julián, Península San Julián, Ría Santa Cruz, Monte León, Río Gallegos (Yorio, 1998); Cabo Blanco, Río Deseado, Bahía Oso Marino, Punta Buque (Gandini \& Frere, 1998a); Ría Santa Cruz, Monte León, Isla Deseada (Frere \& Gandini, 1998); Monte Loayza, Cabo Blanco, Isla Pingüino, Punta Buque, Isla Leones, Isla de Monte León, Isla Deseada (Yorio et al., 2005); Río Deseado, Isla Deseada (Coconier, 2005). Museum specimens. Bahía del Fondo, 6 f, 3 and 4 Jan 1922, col. A. Merkle, MLP; Puerto Deseado, 1 m, 9 Feb 1914, col. S. Shipton, FML, 1 f, 22 Nov 1961, col. A. Zapata, MACN; Río Gallegos, 1 m, 20 Feb 1914, col. S. Shipton, FML.

Field observations. Ría Gallegos, Cabo Vírgenes, Ría Deseado, Punta Loyola, Ría Coig, Estancia Cabo Buen Tiempo, Ría Santa Cruz, Bahía San Julián, Monte León (Imberti).

\section{Larus pipixcan}

Literature. Estuary of the Río Gallegos (Imberti, 2003)

Field observations. Ría Gallegos (Imberti).

\section{Larus atlanticus}

Literature. Puerto Deseado (Olrog, 1948; Savigny et al., 2005); Río Chico (Imberti, 2003); 
Río Gallegos (Coconier, 2005); Río Gallegos, Bahía San Julián (Di Giácomo et al., 2005).

Museum specimens. Caleta Olivia, 1 f, 6 May 1974, col. M. Rumboll, MACN.

\section{Larus dominicanus}

Literature. Río Santa Cruz, Cañadón Misioneros (Oustalet, 1891); Isla del Río Deseado (Oates, 1901); Río Gallegos (Scott \& Sharpe, 1910); Bahía del Fondo (Steullet \& Deautier, 1939); Cabo Blanco, Punta de los Pájaros (Zapata, 1965); Puerto Deseado (Hellmayr \& Conover, 1948b; Daneri, 1959; Zapata, 1967; Daciuk, 1977; Lucero, 1982; de la Peña, 1987, 2005; Gandini \& Frere, 1998b; Nasca et al., 2004), Punta Maqueda (Zapata, 1969); Laguna Los Escarchados, Laguna Escondida (Lange, 1981); Reserva de Vida Silvestre Los Escarchados (Erize, 1983); Cabo Vírgenes, Punta Dungeness (Goodall et al., 1991); Ría Deseado (Albrieu \& Navarro, 1997); Isla Deseada (Arrighi \& Navarro, 1998), Monte Loayza, Cabo Blanco, Ría Deseado, Bahía Oso Marino, Punta Medanosa, Bahía Laura, Bahía San Julián, Península San Julián, Ría Santa Cruz, Monte León, Ría Coig, Río Gallegos, Cabo Vírgenes (Yorio, 1998); Bahía Sanguinetto, Cabo Blanco, Río Deseado, Bahía Oso Marino, Punta Buque (Gandini \& Frere, 1998a); Bahía Laura, Bahía San Julián, Monte León, Isla Deseada, Cabo Vírgenes (Frere \& Gandini, 1998); Monte Loayza, Cabo Blanco, Ría Deseado, Isla Pingüino, Punta Buque, Bahía Laura, Bahía San Julián, Isla Leones, Isla de Monte León, Isla Deseada, Cabo Vírgenes (Yorio et al., 1998); Puerto Deseado (Frere et al., 2000); Laguna Nimez (Imberti \& Albrieu, 2001); Monte Loayza, Cabo Blanco, Ría Deseado, Isla de los Pájaros, Isla Pingüino, Punta Buque, Bahía Laura, Bahía San Julián, Isla Leones, Isla de Monte León, Isla Deseada, Cabo Vírgenes (Yorio et al., 2005); Parque Nacional Los Glaciares (Imberti, 2005); Río Deseado. Isla Deseada (Coconier, 2005).

Museum specimens. Bahía del Fondo, 2 m, 16 Dec 1921, 4 Jan 1922, col. A. Merkle, MLP; Caleta Olivia, 1 ?, 6 May 1974, col. M. Rumboll and E. Shaw; Puerto Deseado, 1 m, 19 Feb 1914, col. S. Shipton, FML, 1 f, 18 Dec 1915, col. J. Mogensen, 1 m, 14 Nov 1961, col. A. Zapata, MACN; Río Gallegos, $1 \mathrm{~m}$ and $1 \mathrm{f}, 20 \mathrm{Feb}$ 1914, col. S. Shipton, FML; 1m, 25 Jul 1969, col. G. A. Voss, USNM.

Field observations. Monumento Natural Bosques Petrificados, Parque Nacional Perito Moreno, Parque Nacional Los Glaciares, Lago Posadas, Estancia Rincón de los Morros, Ría Deseado, Punta Loyola, Cabo Vírgenes, Río Gallegos, Ría Gallegos, Estancia Rupai Pacha, Puerto Bandera, Estancia Bella Vista Bitsch,
Estancia La Soledad, El Zurdo, Estancia El Cóndor, Laguna Los Escarchados, Estancia Sofía, Estancia Pali Aike, Ría Coig, Estancia Cabo Buen Tiempo, Monte León, Estancia La Alice, Estancia Morro Chico, Ría Santa Cruz, Bahía San Julián, El Roda, Estancia La Angostura, Estancia Bella Vista (Imberti).

\section{Sterna maxima maxima}

Literature. Monte Loayza (Yorio, 1998); Ría Deseado (Gandini \& Frere, 1998b); Punta de los Pájaros (Yorio, 2005).

\section{Sterna sandvicensis eurygnatha}

Literature. Puerto Deseado (Saunders, 1896; Junge \& Voous, 1955; Gandini \& Frere, 1998b; Nasca et al., 2004); Punta de los Pájaros (Zapata, 1965; Zapata, 1969); Monte Loayza (Yorio, 1998); Punta de los Pájaros, Bahía Sanguinetto, Río Deseado (Gandini \& Frere, 1998a); Punta de los Pájaros, Monte Loayza (Yorio, 2005).

Field observations. Cabo Vírgenes, Bahía Laura (Imberti); Cabo Blanco (Earnshaw pers. comm.).

\section{Sterna hirundinacea}

Literature. Cañadón Misioneros, Isla Leones (Oustalet, 1891); Puerto Santa Cruz (Saunders, 1896); Isla Leones (Hartert \& Venturi, 1909); Puerto Deseado (Hellmayr, 1932; Hellmayr \& Conover, 1948b; Daciuk, 1977; Gandini \& Frere, 1998b; Nasca et al., 2004); Punta de los Pájaros (Zapata, 1965); Caleta Olivia, Punta de los Pájaros (Zapata, 1969); Monte Loayza, Ría Deseado, Bahía San Julián, Península San Julián, Monte León, Cabo Vírgenes (Yorio, 1998); Caleta Olivia, Punta de los Pájaros, Bahía Sanguinetto, Río Deseado, Bahía Oso Marino, Punta Médano Negro (Gandini \& Frere, 1998a); Monte León (Frere \& Gandini, 1998); Laguna Nimez (Imberti \& Albrieu, 2001); Caleta Olivia, Punta de los Pájaros, Monte Loayza, Isla Pingüino, Bahía Oso Marino, Monte León (Yorio, 2005); Parque Nacional Los Glaciares (Puerto Bandera, Lago Argentino, Lago Roca (Imberti, 2005); Río Gallegos, Monte León (Coconier, 2005); Estancia La Angostura (Imberti, unpubl. b).

Museum specimens. Puerto Deseado, $1 \mathrm{~m}, 9 \mathrm{Feb}$ 1914, 5 ?, 1 Jan 1916, col. S. Shipton, FML, 1 f, 15 Dec 1915, col. J. Mogensen; 1 f, skeleton, 10 Oct 1979, col. P.S. Humphrey, MACN; Puerto Medanoso, southern Puerto Deseado, 1 m, 19 Sep 1972, col. C. Olrog, FML.

Field observations. Parque Nacional Los Glaciares, Ría Deseado, Ría Gallegos, Ría Coig, Monte León, Puerto Bandera, Estancia La Soledad, Estancia Cabo Buen Tiempo, Cabo Vírgenes, Punta Loyola, El Roda (Imberti). 
Rynchops niger cinerascens

Literature. Puerto Santa Cruz (Humphrey \& Bridge, 1970).

Field observations. Ría Gallegos (Ferrari and Albrieu pers. comm.).

\section{COLUMBIDAE}

\section{Columba livia livia}

Literature. Puerto Deseado (Zapata, 1967; Daciuk, 1977), Estancia El Cuadro (De Lucca \& Saggese, 1992); Laguna Nimez (Imberti \& Albrieu, 2001); Parque Nacional Los Glaciares (El Chaltén and Lago Roca) (Imberti, 2005); Estancia La Angostura (Imberti, unpubl. b).

Field observations. Monumento Natural Bosques Petrificados, Parque Nacional Los Glaciares, Río Gallegos, Ría Gallegos, Estancia Rincón de los Morros, Santa Cruz (Imberti).

\section{Columba araucana}

Literature. Lago Roca (Fjelså \& Krabbe, 1990); Chorrillo del Salto (Administración de Parques Nacionales 1994-97); Parque Nacional Los Glaciares (Lago Roca, El Chaltén), El Zurdo (Imberti, 2003); El Zurdo, Parque Nacional Los Glaciares, Estancia La Soledad, Estancia La Anita (Di Giácomo et al., 2005).

Field observations. El Zurdo, Lago Roca (Imberti); Calafate (Zoratti pers. comm.).

\section{Zenaida auriculata auriculata}

Literature. Río Chico, Santa Cruz (Scott \& Sharpe, 1904); Bahía del Fondo (Steullet \& Deautier, 1939); Río Gallegos (Hellmayr \& Conover, 1942); Puerto Deseado (Zapata, 1967; Daciuk, 1977), Punta Murphy (Zapata, 1969); Laguna Los Escarchados (Lange, 1981); Estancia El Cuadro, Monumento Natural Bosques Petrificados (De Lucca \& Saggese, 1992); Laguna Nimez (Imberti \& Albrieu, 2001); Parque Nacional Los Glaciares (Imberti, 2005), Cabo Vírgenes (Imberti, unpubl. a).

Museum specimens. Bahía del Fondo, 1 f, 28 Dec 1921, col. A. Merkle, MLP; Estancia Roca Blanca, 4 f, 19 and 23 Dec 1960, col. A, Budin and J. Viera, FML; Lago Argentino, Lago Roca, 1 f, 26 Jan 1959, col. P. Miles, MACN.

Field observations. Monumento Natural Bosques Petrificados, Parque Nacional Los Glaciares, Parque Nacional Perito Moreno, Lago Posadas, Río Gallegos, Estancia Rincón de los Morros, Estancia Rupai Pacha, Estancia 9 de Julio, Laguna Los Escarchados, Meseta Lago Strobel, Puerto Bandera, Estancia La Soledad, Estancia La Angostura, Ría Coig, El Roda (Imberti).

\section{Columbina picui picui}

Literature. Estancia Alta Vista (Chebez et al., 1988).

\section{Metriopelia melanoptera melanoptera}

Literature. Between Bahía del Túnel and Río Mitre (Imberti, 2003); near Glaciar Perito Moreno, Paraje Punta del Monte, Estancia Cancha Carrera (Imberti, 2005); Meseta Lago Strobel, Parque Nacional Perito Moreno, Meseta Lago Buenos Aires, El Zurdo, Meseta del Asador, Parque Nacional Los Glaciares, Estancia La Soledad, Estancia La Anita (Di Giácomo et al., 2005); Estancia La Angostura (Imberti, unpubl. b).

Field observations. Parque Nacional Los Glaciares, Estancia La Quinta (Imberti); Parque Nacional Perito Moreno (M. Martínez pers. comm.)

\section{PSITTACIDAE}

\section{Cyanoliseus patagonus patagonus}

Literature. Río Chico (Stone, 1928); Bahía del Fondo, Río Gallegos (Steullet \& Deautier, 1945; Darrieu, 1980); Monumento Natural Bosques Petrificados, Estancia El Cuadro (Di Giácomo et al., 2005). Museum specimens. Bahía del Fondo, $1 \mathrm{~m}$ and 1 f, 19 Dec 1921, col. A. Merkle; Río Gallegos, 1 f, Aug 1894, collector unknown, MLP.

Field observations. Monumento Natural Bosques Petrificados (Imberti).

\section{Enicognathus ferrugineus minor}

Literature. Río Chico (Chapman, 1919; Stone, 1928); Lago Argentino (Orfila, 1937; Steullet \& Deautier, 1945); Lago Argentino, Lago San Martín (Darrieu, 1982); Parque Nacional Los Glaciares (Imberti, 2005); Parque Nacional Perito Moreno, Laguna Nimez, Lago Argentino, Parque Nacional Los Glaciares, Estancia La Soledad, Estancia La Anita (Di Giácomo et al., 2005).

Museum specimens. Lago Argentino, $1 \mathrm{~m}, \mathrm{Feb}$ 1928, col. E. Budin; 1 ?, Jan 1959, col. P. Miles; Lago San Martín, 1?, 3 Aug 1936, col. S. Radboone, MACN.

Field observations. Parque Nacional Perito Moreno, Parque Nacional Los Glaciares, Lago Posadas, Lago del Desierto, Estancia Cóndor, Estancia Rincón de los Morros, Estancia Glencross, Estancia Los Chorrillos (Imberti).

\section{TYTONIDAE}

\section{Tyto alba tuidara}

Literature. Río Chico (Scott \& Sharpe, 1915); Estancia El Cuadro, Monumento Natural Bosques 
Petrificados (De Lucca \& Saggese, 1992); Parque Nacional Los Glaciares (El Chaltén, Lago Argentino, Glaciar Perito Moreno, Río Mitre (Imberti, 2005), Cabo Vírgenes (Imberti, unpubl. a); Estancia La Angostura (Imberti, unpubl. b).

Field observations. Parque Nacional Perito Moreno, Parque Nacional Los Glaciares, Monumento Natural Bosques Petrificados, Ría Gallegos (Imberti).

\section{STRIGIDAE}

\section{Bubo magellanicus}

Literature. Río Ecker, Río Gallegos (Scott \& Sharpe, 1915); Puerto Deseado (Burmeister, 1890; Oustalet, 1891); Río Gallegos (Anónimo, 1924; Serié, 1926), Estancia El Cuadro, Monumento Natural Bosques Petrificados (De Lucca \& Saggese, 1992); Parque Nacional Los Glaciares (Imberti, 2005), Cabo Vírgenes (Imberti, unpubl. a).

Museum specimens. Las Heras, Estancia Roca Blanca, 1 ?, 22 Dec 1960, col. O. Budin and J. C.Viera, FML.

Field observations. Parque Nacional Perito Moreno, Parque Nacional Los Glaciares, Monumento Natural Bosques Petrificados, Estancia Rincón de los Morros, Estancia La Angostura, Estancia Anita, Estancia 9 de Julio, Estancia Cabo Buen Tiempo (Imberti).

\section{Strix rufipes rufipes}

Literature. Río Chico (Scott \& Sharpe, 1915); Puerto Deseado (Burmeister, 1890); Parque Nacional Los Glaciares (Imberti, 2005).

Field observations. Parque Nacional Los Glaciares (Imberti); Parque Nacional Perito Moreno (M. Martínez pers. comm.).

\section{Glaucidium nanum}

Literature. Río Chico (Scott \& Sharpe, 1915); Lago Argentino, Lago Roca, Parque Nacional Los Glaciares (König, 1991); Parque Nacional Los Glaciares (Imberti, 2005); Estancia La Angostura (Imberti, unpubl. b).

Museum specimens. Lago Argentino, Cerro Mayo, 1 f, 14 Feb 1959, col. P. Miles, MACN.

Field observations. Parque Nacional Perito Moreno, Parque Nacional Los Glaciares, Estancia Rincón de los Morros (Imberti).

\section{Atthene cunicularia partridgei}

Literature. Río Chico (Scott \& Sharpe, 1915); Cañadón Seco, Cañadón León, El Cordón -near Caleta Olivia and Piedra Clavada- (Navas \& Bó, 1997), Cabo Vírgenes (Imberti, unpunl. a); Estancia La Angostura (Imberti, unpubl. b).
Field observations. Monumento Natural Bosques Petrificados, Río Pinturas, Ría Gallegos (Imberti).

\section{Asio flammeus suinda}

Literature. Between Lago Roca and El Calafate (Imberti, 2005), Cabo Vírgenes (Imberti, unpubl. a); Estancia La Angostura (Imberti, unpubl. b).

Field observations. Parque Nacional Perito Moreno, Parque Nacional Los Glaciares, Laguna Los Escarchados, Estancia Cerro Ventana (Imberti).

\section{CAPRIMULGIDAE}

\section{Podager nacunda nacunda}

Literature. Estancia Rincón de Los Morros (Imberti, 2001)

Field observations. Estancia Rincón de los Morros (Imberti).

\section{Caprimulgus longirostris patagonicus}

Literature. Estancia El Tranquilo, $250 \mathrm{~km}$ northeastern of San Julián (Olrog, 1962) Punta Murphy, Fitz Roy (Zapata, 1969) Estancia El Cuadro (De Lucca \& Saggese, 1992); Parque Nacional Los Glaciares (Imberti, 2005).

Museum specimens. Estancia El Tranquilo, Depto. Magallanes, $4 \mathrm{~m}, 2 \mathrm{f}$ and 1 ?, 19-22 Jan 1961; Estancia Roca Blanca, 1 m, 17 Jan 1961, col. A. Budin and J. Viera, FML.

Field observations. Parque Nacional Perito Moreno, Parque Nacional Los Glaciares, Monumento Natural Bosques Petrificados (Imberti).

\section{TROCHILIDAE}

\section{Oreotrochilus leucopleurus}

Literature. Lago San Martín (Serret \& Johnson, 1986), Parque Nacional Perito Moreno, Parque Nacional Los Glaciares (Chebez et al., 1998) El Chaltén, Parque Nacional Los Glaciares (Mazar Barnett, 2001); Parque Nacional Los Glaciares (Cordón Mascarello, Estancia Helsingfors) (Imberti, 2005); Parque Nacional Perito Moreno, Parque Nacional Los Glaciares, Estancia La Soledad, Estancia La Anita (Di Giácomo et al., 2005).

Field observations. Parque Nacional Perito Moreno (Imberti), Parque Nacional Los Glaciares (Mazar Barnett pers. comm.).

\section{Sephanoides sephaniodes}

Literature. Cabo Buen Tiempo (Stone, 1928); Parque Nacional Los Glaciares (Imberti, 2005).

Field observations. Parque Nacional Los Glaciares, Estancia Bella Vista (Imberti); Río Gallegos (Sturzenbaum pers. comm.). 


\section{ALCEDINIDAE}

\section{Megaceryle torquata stellata}

Literature. Between Río Mitre and Parque Nacional Los Glaciares (Imberti, 2005)

\section{PICIDAE}

\section{Picoides lignarius}

Literature. Parque Nacional Los Glaciares (Lago Roca, El Chaltén), Bahía Onelli (Imberti, 2005); Parque Nacional Perito Moreno, Parque Nacional Los Glaciares, Estancia La Soledad, Estancia La Anita (Di Giácomo et al., 2005).

Field observations. Parque Nacional Perito Moreno, Parque Nacional Los Glaciares, Estancia Rincón de los Morros, Estancia Glencross (Imberti).

\section{Colaptes pitius}

Literature. Río Chico (Stone, 1928); Lago San Martín (Pergolani de Costa, 1942); Parque Nacional Los Glaciares (Imberti, 2005).

Museum specimens. Lago Argentino, Punta Bandera, 1 m, 24 Feb 1959, col. P. Miles, MACN. Field observations. Parque Nacional Perito Moreno, Parque Nacional Los Glaciares, Estancia Rincón de los Morros, Lago Posadas, Estancia Cóndor, Estancia La Maipú, Estancia Anita, Estancia Glencross, Estancia La Alice (Imberti).

\section{Campephilus magellanicus}

Literature. Parque Nacional Los Glaciares (Imberti, 2005); Parque Nacional Perito Moreno, Parque Nacional Los Glaciares, Estancia La Soledad, Estancia La Anita (Di Giácomo et al., 2005).

Appendix 1.

87 Aguada Grande (= Estancia Aguada Grande) $\left(50^{\circ} 15^{\prime} \mathrm{S}-69^{\circ} 38^{\prime} \mathrm{W}\right)$

4 Bahía del Fondo (= Caleta del Fondo) $\left(46^{\circ} 06^{\prime} \mathrm{S}\right.$ $67^{\circ} 36^{\prime} \mathrm{W}$ )

64 Bahía del Túnel (ca. $49^{\circ} 20^{\prime} \mathrm{S}-72^{\circ} 55^{\prime} \mathrm{W}$ )

43 Bahía de los Nodales (ca. $47^{\circ} 58^{\prime} \mathrm{S}$ - $65^{\circ} 55^{\prime} \mathrm{W}$ )

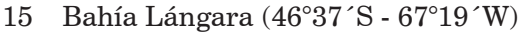

48 Bahía Laura $\left(48^{\circ} 21^{\prime} \mathrm{S}-66^{\circ} 21^{\prime} \mathrm{W}\right)$

136 Bahía Onelli (ca. $50^{\circ} 10^{\prime} \mathrm{S}-73^{\circ} 05^{\prime} \mathrm{W}$ )

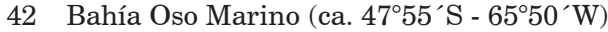

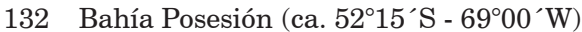

85 Bahía Redonda $\left(50^{\circ} 19^{\prime} \mathrm{S}-72^{\circ} 16^{\prime} \mathrm{W}\right)$

31 Bahía Sanguinetto $\left(47^{\circ} 08^{\prime} \mathrm{S}-66^{\circ} 00^{\prime} \mathrm{W}\right)$

63 Bahía San Julián $\left(49^{\circ} 21^{\prime} \mathrm{S}-67^{\circ} 42^{\prime} \mathrm{W}\right)$

89 Bahía Viento Norte $\left(50^{\circ} 28^{\prime} \mathrm{S}-73^{\circ} 01^{\prime} \mathrm{W}\right)$

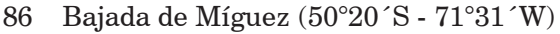

Museum specimens. Lago Argentino, Ventisquero Onelli, 1 m, 7 Jan 1959, col. P. Miles, MACN. Field observations. Parque Nacional Perito Moreno, Parque Nacional Los Glaciares, Estancia Cóndor (Imberti).

\section{CONCLUSIONS}

The study of literature, museum specimens and field observations permitted us establish that Anatidae (24 spp), Scolopacidae (14 spp), Laridae (14 spp), Accipitridae (8 spp), Procellariidae (8 spp) and Falconidae (7 spp) are the best represented families in Santa Cruz Province.

Based on field observations, the first precise localities are presented for Aptenodytes patagonicus, Diomedea exulans, Procellaria aequinoctialis, Puffinus puffinus, Puffinus gravis, Chloephaga hybrida, Heteronetta atricapilla, Buteo magnirostris and Porzana spiloptera.

New localities are provided for 114 species.

\section{ACKNOWLEDGEDMENTS}

For allowing us access to, or supplying specimen data on the collection in their care, we extend thanks to Jorge R. Navas, Pablo Tubaro and Yolanda Davies (MACN), and Ada L. Echevarría (FML). The following persons provided information on their own field observations: C. Albrieu, L. Bernacchi, A. Earnshaw, J. Fenton, S. Ferrari, N. Gardner, G. Harris, S. Howell, M. Martínez, J. Mazar Barnett, M. McNamara, M. Pearman, H. Rodríguez Goñi, B. Roil, P. and S. Sturzenbaum, S. Webb, J. Zancaner and C. Zoratti. We thank Cecilia Gorretta for the map and Lucas Marti for the English translation.
27 Bajo Caracoles (= Caracoles) $\left(47^{\circ} 27^{\prime} \mathrm{S}-70^{\circ} 57^{\prime} \mathrm{W}\right)$

119 Baliza Población $\left(51^{\circ} 37^{\prime} \mathrm{S}-69^{\circ} 12^{\prime} \mathrm{W}\right)$

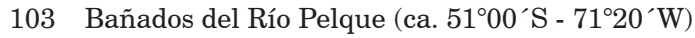

103 Bañados del Tero (ca. $51^{\circ} 00 \mathrm{~S}-71^{\circ} 20^{\prime} \mathrm{W}$ )

69 Barranca Blanca $\left(49^{\circ} 35^{\prime} \mathrm{S}-67^{\circ} 59^{\prime} \mathrm{W}\right)$

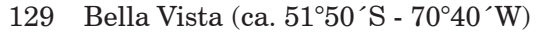

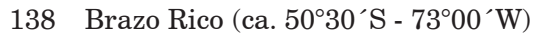

33 Cabo Blanco $\left(47^{\circ} 12^{\prime} \mathrm{S}-65^{\circ} 45^{\prime} \mathrm{W}\right)$

117 Cabo Buen Tiempo (51 $33^{\prime} \mathrm{S}-68^{\circ} 57^{\prime} \mathrm{W}$ )

32 Cabo Tres Puntas $\left(47^{\circ} 06^{\prime} \mathrm{S}-65^{\circ} 33^{\prime} \mathrm{W}\right)$

134 Cabo Vírgenes $\left(52^{\circ} 20^{\prime} \mathrm{S}-68^{\circ} 21^{\prime} \mathrm{W}\right)$

14 Caleta Olivia (46은 $\left.{ }^{\circ} \mathrm{S}-67^{\circ} 32^{\prime} \mathrm{W}\right)$

108 Cancha Carrera ( $\left.51^{\circ} 15^{\prime} \mathrm{S}-72^{\circ} 14^{\prime} \mathrm{W}\right)$

94 Cañadón de las Vacas $\left(50^{\circ} 31^{\prime} \mathrm{S}-69^{\circ} 10^{\prime} \mathrm{W}\right)$

41 Cañadón del Puerto $\left(47^{\circ} 45^{\prime} \mathrm{S}-66^{\circ} 00^{\prime} \mathrm{W}\right)$

131 Cañadón Gapp (52 $\left.12^{\circ} \mathrm{S}-68^{\circ} 34^{\prime} \mathrm{W}\right)$

15 Cañadón León (46 $\left.37^{\circ} \mathrm{S}-67^{\circ} 21^{\prime} \mathrm{W}\right)$

81 Cañadón Misioneros (ca. 5001'S - 68 $31^{\circ} \mathrm{W}$ ) 
14 Cañadón Seco (ca. $46^{\circ} 26^{\prime} \mathrm{S}-67^{\circ} 32^{`} \mathrm{~W}$ )

3 Caprek-aik $\left(46^{\circ} 01^{\prime} \mathrm{S}-69^{\circ} 20^{\prime} \mathrm{W}\right)$

98 Cerrito (= El Cerrito) $\left(50^{\circ} 39^{\prime} \mathrm{S}-71^{\circ} 24^{\prime} \mathrm{W}\right)$

49 Cerro Amette $\left(48^{\circ} 28^{\prime} \mathrm{S}-66^{\circ} 42^{\prime} \mathrm{W}\right)$

1 Cerro Colorado (ca. $46^{\circ} 50^{\prime} \mathrm{S}-71^{\circ} 50^{\prime} \mathrm{W}$ )

139 Cerro Comisión de Elefantes (50²0`S - 72³0`W) Cerro de la Picana (not localized)

113 Cerro Dorotea (ca. $51^{\circ} 35^{\prime} \mathrm{S}-72^{\circ} 20^{\prime} \mathrm{W}$ )

75 Cerro Fortaleza (ca. $50^{\circ} 11^{\prime} \mathrm{S}-71^{\circ} 17^{\prime} \mathrm{W}$ )

137 Cerro Mayo (ca. $50^{\circ} 20^{\prime} \mathrm{S}-73^{\circ} 10^{\prime} \mathrm{W}$ )

12 Cerro Mojón (ca. $46^{\circ} 33^{\prime} \mathrm{S}-68^{\circ} 57^{\prime} \mathrm{W}$ )

85 Cerro Moreno (ca. $50^{\circ} 13^{\prime} \mathrm{S}-72^{\circ} 25^{\prime} \mathrm{W}$ )

101 Cerro Observatorio $\left(50^{\circ} 39^{\prime} \mathrm{S}-69^{\circ} 09^{\prime} \mathrm{W}\right)$

19 Cerro Rosado $\left(47^{\circ} 04^{\prime} \mathrm{S}-70^{\circ} 35^{\prime} \mathrm{W}\right)$

93 Cheike (possibly Estancia Chall Aike: 50³0`S $\left.70^{\circ} 50^{\prime} \mathrm{W}\right)$

68 Chonque Aike $\left(49^{\circ} 24^{\prime} \mathrm{S}-69^{\circ} 32^{\prime} \mathrm{W}\right)$

64 Chorrillo del Salto (ca. $49^{\circ} 20^{\prime} \mathrm{S}-72^{\circ} 55^{\prime} \mathrm{W}$ )

82 Comandante Luis Piedra Buena $\left(49^{\circ} 59^{\prime} \mathrm{S}\right.$ $68^{\circ} 54^{`} \mathrm{~W}$ )

66 Cordón Mascarello (49 $\left.35^{\prime} \mathrm{S}-72^{\circ} 35^{\prime} \mathrm{W}\right)$

100 Coti Aike (= Puesto Coti Aike) (ca. 5044’S $\left.71^{\circ} 30^{\prime} \mathrm{W}\right)$

90 Ea. 9 de Julio $\left(50^{\circ} 23^{\prime} \mathrm{S}-72^{\circ} 43^{\prime} \mathrm{W}\right)$

140 Ea. Achalay $\left(50^{\circ} 43^{\prime} \mathrm{S}-71^{\circ} 55^{\prime} \mathrm{W}\right)$

95 Ea. Alta Vista (50 $\left.28^{\prime} \mathrm{S}-72^{\circ} 36^{\prime} \mathrm{W}\right)$

85 Ea. Anita (ca. $50^{\circ} 23^{\prime} \mathrm{S}-72^{\circ} 43^{\prime} \mathrm{W}$ )

62 Ea. Arbilla $\left(49^{\circ} 12^{\prime} \mathrm{S}-72^{\circ} 55^{\prime} \mathrm{W}\right)$

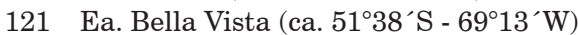

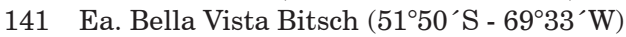

142 Ea. Buitreras (51 $\left.44^{\prime} \mathrm{S}-70^{\circ} 08^{\prime} \mathrm{W}\right)$

117 Ea. Cabo Buen Tiempo (ca. 51 $33^{\prime} \mathrm{S}-68^{\circ} 57^{\prime} \mathrm{W}$ )

Ea. Cañadón del Chara (not localized)

11 Ea. Cañadón Vasco $\left(46^{\circ} 38^{\prime} \mathrm{S}-69^{\circ} 13^{\prime} \mathrm{W}\right)$

143 Ea. Cerro Ventana $\left(49^{\circ} 00^{\prime} \mathrm{S}-70^{\circ} 16^{\prime} \mathrm{W}\right)$

144 Ea. Cóndor $\left(52^{\circ} 09^{\prime} \mathrm{S}-69^{\circ} 04^{\prime} \mathrm{W}\right)$

106 Ea. Coy Inlet $\left(50^{\circ} 58^{\prime} \mathrm{S}-69^{\circ} 13^{\prime} \mathrm{W}\right)$

71 Ea. Cristina $\left(50^{\circ} 01^{\prime} \mathrm{S}-73^{\circ} 06^{\prime} \mathrm{W}\right)$

145 Ea. Don Braulio (51 $\left.43^{\circ} \mathrm{S}-69^{\circ} 17^{\prime} \mathrm{W}\right)$

132 Ea. El Cóndor (= El Cóndor) $\left(48^{\circ} 56^{\prime} \mathrm{S}-72^{\circ} 39^{\prime} \mathrm{W}\right)$

30 Ea. El Cuadro (47 $\left.30^{\prime} \mathrm{S}-68^{\circ} 00^{\prime} \mathrm{W}\right)$

28 Ea. El Milagro (ca. $47^{\circ} 25^{\prime} \mathrm{S}-70^{\circ} 56^{\prime} \mathrm{W}$ )

102 Ea. El Tero $\left(50^{\circ} 48^{\prime} \mathrm{S}-71^{\circ} 32^{\prime} \mathrm{W}\right)$

74 Ea. El Tranquilo $\left(50^{\circ} 08^{\prime} \mathrm{S}-71^{\circ} 41^{\prime} \mathrm{W}\right)$

125 Ea. Glencross $\left(51^{\circ} 50^{\prime} \mathrm{S}-71^{\circ} 33^{\prime} \mathrm{W}\right)$

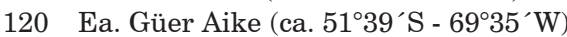

66 Ea. Helsingfors (ca. $49^{\circ} 35^{\prime} \mathrm{S}-72^{\circ} 35^{\prime} \mathrm{W}$ )

116 Ea. Killik Aike Norte (51 $34^{\circ} \mathrm{S}-69^{\circ} 28^{\prime} \mathrm{W}$ )

116 Ea. Killik Aike Sur (ca, 51 $31^{\circ} 36^{\prime} \mathrm{S}-69^{\circ} 35^{\prime} \mathrm{W}$ )

146 Ea. La Alice (50²0'S - 70³2'W)

109 Ea. La Angelina (51 $\left.25^{\prime} \mathrm{S}-69^{\circ} 04^{\prime} \mathrm{W}\right)$

52 Ea. La Angostura (48 $\left.37^{\prime} \mathrm{S}-70^{\circ} 39^{\prime} \mathrm{W}\right)$

120 Ea. La Carlota $\left(51^{\circ} 39^{\prime} \mathrm{S}-69^{\circ} 35^{\prime} \mathrm{W}\right)$

60 Ea. La Carreta (48 $\left.57^{\prime} \mathrm{S}-70^{\circ} 12^{\prime} \mathrm{W}\right)$

34 Ea. La Estrella $\left(47^{\circ} 19^{\prime} \mathrm{S}-65^{\circ} 57^{\prime} \mathrm{W}\right)$

58 Ea. La Federica $\left(49^{\circ} 00^{\prime} \mathrm{S}-72^{\circ} 10^{\prime} \mathrm{W}\right)$

Ea. La Frontera (not localized)

147 Ea. La Lucha $\left(48^{\circ} 29^{\prime} \mathrm{S}-70^{\circ} 26^{\prime} \mathrm{W}\right)$

148 Ea. La Maipú $\left(49^{\circ} 08^{\prime} \mathrm{S}-72^{\circ} 30^{\prime} \mathrm{W}\right)$

149 Ea. La Nélida $\left(50^{\circ} 59^{\prime} \mathrm{S}-71^{\circ} 25^{\prime} \mathrm{W}\right)$

21 Ea. La Querencia $\left(47^{\circ} 01^{\prime} \mathrm{S}-67^{\circ} 24^{\prime} \mathrm{W}\right)$

64 Ea. La Quinta (49 $\left.20^{\prime} \mathrm{S}-72^{\circ} 54^{\prime} \mathrm{W}\right)$

20 Ea. La Renania $\left(47^{\circ} 02^{\prime} \mathrm{S}-70^{\circ} 00^{\prime} \mathrm{W}\right)$

150 Ea. La Siberia $\left(48^{\circ} 59^{\prime} \mathrm{S}-71^{\circ} 11^{\prime} \mathrm{W}\right)$
84 Ea. La Soledad $\left(50^{\circ} 20^{\prime} \mathrm{S}-72^{\circ} 47^{\prime} \mathrm{W}\right)$

151 Ea. Las Horquetas $\left(51^{\circ} 22^{\prime} \mathrm{S}-70^{\circ} 14^{\prime} \mathrm{W}\right)$

108 Ea. Las Tres Marías (51 $17^{\circ} \mathrm{S}-72^{\circ} 10^{\prime} \mathrm{W}$ )

59 Ea. Las Tunas (ca. $48^{\circ} 55^{\prime} \mathrm{S}-71^{\circ} 10^{\prime} \mathrm{W}$ )

113 Ea. Los Chorrillos (51 $\left.38^{\circ} \mathrm{S}-72^{\circ} 13^{\prime} \mathrm{W}\right)$

29 Ea. Los Gurises $\left(47^{\circ} 45^{\prime} \mathrm{S}-71^{\circ} 30^{\prime} \mathrm{W}\right)$

152 Ea. Los Luises $\left(50^{\circ} 41^{\prime} \mathrm{S}-69^{\circ} 29^{\prime} \mathrm{W}\right)$

116 Ea. Los Pozos (51 $\left.27^{\prime} \mathrm{S}-69^{\circ} 20^{\prime} \mathrm{W}\right)$

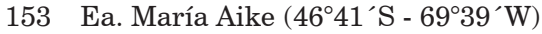

133 Ea. Monte Dinero (ca. 52 ${ }^{\circ} 20^{\prime} \mathrm{S}-68^{\circ} 35^{\prime} \mathrm{W}$ )

154 Ea. Morro Chico $\left(51^{\circ} 57^{\prime} \mathrm{S}-71^{\circ} 33^{\prime} \mathrm{W}\right)$

155 Ea. Moy Aike Chico (51 $\left.09^{\prime} \mathrm{S}-69^{\circ} 40^{\prime} \mathrm{W}\right)$

155 Ea. Moy Aike Grande $\left(51^{\circ} 05^{\prime} \mathrm{S}-69^{\circ} 41^{\prime} \mathrm{W}\right)$

156 Ea. Palermo Aike (51 $\left.41^{\prime} \mathrm{S}-69^{\circ} 43^{\prime} \mathrm{W}\right)$

130 Ea. Pali Aike (51 $\left.58^{\prime} \mathrm{S}-69^{\circ} 42^{\prime} \mathrm{W}\right)$

122 Ea. Punta Alta $\left(51^{\circ} 41^{\prime} \mathrm{S}-71^{\circ} 58^{\prime} \mathrm{W}\right)$

12 Ea. Reconquista (ca. $46^{\circ} 33^{\prime} \mathrm{S}-68^{\circ} 57^{\prime} \mathrm{W}$ )

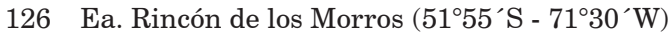

54 Ea. Roca Blanca (ca. $48^{\circ} 40^{\prime} \mathrm{S}-68^{\circ} 50^{\prime} \mathrm{W}$ )

157 Ea. Rupai Pacha $\left(51^{\circ} 02^{\prime} \mathrm{S}-72^{\circ} 04^{\prime} \mathrm{W}\right)$

66 Ea. San Lorenzo (ca. $49^{\circ} 35^{\prime} \mathrm{S}-72^{\circ} 35^{\prime} \mathrm{W}$ )

66 Ea. Santa Margarita (ca. $49^{\circ} 35^{\prime} \mathrm{S}-72^{\circ} 35^{\prime} \mathrm{W}$ )

129 Ea. Sofía $\left(51^{\circ} 57^{\prime} \mathrm{S}-70^{\circ} 49^{\prime} \mathrm{W}\right)$

114 Ea. Stag River $\left(51^{\circ} 39^{\prime} \mathrm{S}-72^{\circ} 01^{\prime} \mathrm{W}\right)$

118 Ea. Tres de Enero (ca. 51 $38^{\circ} \mathrm{S}-69^{\circ} 13^{\prime} \mathrm{W}$ )

158 Ea. Verdadera Argentina (50 $\left.51^{\prime} \mathrm{S}-72^{\circ} 11^{\prime} \mathrm{W}\right)$

91 El Calafate $\left(50^{\circ} 20^{\prime} \mathrm{S}-72^{\circ} 18^{\prime} \mathrm{W}\right)$

64 El Chaltén $\left(49^{\circ} 20^{\prime} \mathrm{S}-72^{\circ} 55^{\prime} \mathrm{W}\right)$

El Cordón (not localized)

159 El Pluma (4630`S - $\left.70^{\circ} 00^{\prime} \mathrm{W}\right)$

60 El Roda $\left(51^{\circ} 18^{\prime} \mathrm{S}-71^{\circ} 46^{\prime} \mathrm{W}\right)$

55 El Salado $\left(48^{\circ} 43^{\prime} \mathrm{S}-67^{\circ} 47^{\prime} \mathrm{W}\right)$

128 El Zurdo $\left(52^{\circ} 00^{\prime} \mathrm{S}-71^{\circ} 15^{\prime} \mathrm{W}\right)$

77 Emelk-aik $\left(49^{\circ} 50^{\prime} \mathrm{S}-68^{\circ} 37^{\prime} \mathrm{W}\right)$

105 Faro Coig $\left(50^{\circ} 52^{\prime} \mathrm{S}-69^{\circ} 09^{\prime} \mathrm{W}\right)$

22 Fitz Roy $\left(47^{\circ} 02^{\prime} \mathrm{S}-67^{\circ} 15^{\prime} \mathrm{W}\right)$

138 Glaciar Perito Moreno (ca. 50 $30^{\circ} \mathrm{S}-73^{\circ} 00^{\prime} \mathrm{W}$ )

53 Gobernador Gregores (48 $46^{\prime} \mathrm{S}-70^{\circ} 15^{\prime} \mathrm{W}$ )

7 Golfo de San Jorge ( $\left.46^{\circ} 18^{\prime} \mathrm{S}-67^{\circ} 43^{\prime} \mathrm{W}\right)$

41 Isla Chaffers $\left(47^{\circ} 46^{\prime} \mathrm{S}-65^{\circ} 52^{\prime} \mathrm{W}\right)$

41 Isla de los Pájaros (ca. $47^{\circ} 45^{\prime} \mathrm{S}-65^{\circ} 54^{\prime} \mathrm{W}$ )

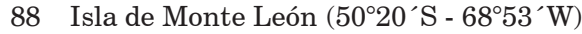

118 Isla Deseada (51 $\left.41^{\circ} \mathrm{S}-69^{\circ} 16^{\prime} \mathrm{W}\right)$

41 Isla Elena $\left(47^{\circ} 45^{\prime} \mathrm{S}-65^{\circ} 56^{\prime} \mathrm{W}\right)$

43 Isla Guano (48 $\left.00^{\prime} \mathrm{S}-65^{\circ} 54^{\prime} \mathrm{W}\right)$

80 Isla Leones $\left(50^{\circ} 04^{\prime} \mathrm{S}-68^{\circ} 26^{\prime} \mathrm{W}\right)$

43 Isla Lobos $\left(47^{\circ} 54^{\prime} \mathrm{S}-65^{\circ} 53^{\prime} \mathrm{W}\right)$

82 Isla Pavón $\left(50^{\circ} 00^{\prime} \mathrm{S}-68^{\circ} 55^{\prime} \mathrm{W}\right)$

43 Isla Pingüino (= Isla de los Pingüinos) $\left(47^{\circ} 53^{\prime} \mathrm{S}\right.$ $\left.-65^{\circ} 49^{\prime} \mathrm{W}\right)$

41 Isla Quiroga (ca. $47^{\circ} 45^{\prime} \mathrm{S}-65^{\circ} 54 \mathrm{~W}$ )

56 Islote Chato $\left(48^{\circ} 44^{\prime} \mathrm{S}-67^{\circ} 03^{\prime} \mathrm{W}\right)$

45 Islote del Cabo $\left(48^{\circ} 15^{\prime} \mathrm{S}-66^{\circ} 16^{\prime} \mathrm{W}\right)$

111 La Esperanza (ca. $51^{\circ} 20^{\prime} \mathrm{S}-72^{\circ} 05^{\prime} \mathrm{W}$ )

164 La Manchuria (ca. $48^{\circ} 04^{\prime} \mathrm{S}-69^{\circ} 55^{\prime} \mathrm{W}$ )

150 La Siberia $\left(48^{\circ} 59^{\prime} \mathrm{S}-71^{\circ} 11^{\prime} \mathrm{W}\right)$

85 Lago Argentino (50 $\left.10^{\circ} \mathrm{S}-72^{\circ} 25^{\prime} \mathrm{W}\right)$

37 Lago Belgrano (47 $50^{\circ} \mathrm{S}-72^{\circ} 09^{\prime} \mathrm{W}$ )

5 Lago Buenos Aires (46 $\left.30^{\circ} \mathrm{S}-72^{\circ} 00^{\prime} \mathrm{W}\right)$

38 Lago Burmeister $\left(47^{\circ} 57^{\prime} \mathrm{S}-72^{\circ} 09^{\prime} \mathrm{W}\right)$

59 Lago Cardiel (ca. $48^{\circ} 55^{\prime} \mathrm{S}-71^{\circ} 10^{\prime} \mathrm{W}$ )

161 Lago Cardiel Chico (48 $\left.55^{\prime} \mathrm{S}-71^{\circ} 52^{\prime} \mathrm{W}\right)$

61 Lago del Desierto $\left(49^{\circ} 02^{\prime} \mathrm{S}-72^{\circ} 58^{\prime} \mathrm{W}\right)$

96 Lago Frío (ca. $50^{\circ} 20^{\prime} \mathrm{S}-72^{\circ} 18^{\prime} \mathrm{W}$ ) 
128 Lago Little Hill ( = Laguna Little Hill) $\left(51^{\circ} 55^{\prime} \mathrm{S}\right.$ $-71^{\circ} 19^{\prime} \mathrm{W}$ )

26 Lago Posadas (ca. $47^{\circ} 30^{\prime} \mathrm{S}-71^{\circ} 50^{\prime} \mathrm{W}$ )

25 Lago Pueyrredón $\left(47^{\circ} 15^{\prime} \mathrm{S}-72^{\circ} 05^{\prime} \mathrm{W}\right)$

46 Lago Quiroga (ca. $48^{\circ} 20^{\prime} \mathrm{S}-71^{\circ} 30^{\prime} \mathrm{W}$ )

99 Lago Roca (ca. $50^{\circ} 30^{\prime} \mathrm{S}-72^{\circ} 40^{\prime} \mathrm{W}$ )

57 Lago San Martín (48 $\left.52^{\circ} \mathrm{S}-72^{\circ} 25^{\prime} \mathrm{W}\right)$

66 Lago Viedma $\left(49^{\circ} 35^{\prime} \mathrm{S}-72^{\circ} 35^{\prime} \mathrm{W}\right)$

162 Lagos del Furioso $\left(47^{\circ} 28^{\prime} \mathrm{S}-71^{\circ} 47^{\prime} \mathrm{W}\right)$

163 Laguna Azul (52 $\left.04^{\prime} \mathrm{S}-69^{\circ} 34^{\prime} \mathrm{W}\right)$ Laguna Balton (not localized)

123 Laguna Cóndor $\left(49^{\circ} 09^{\prime} \mathrm{S}-72^{\circ} 57^{\prime} \mathrm{W}\right)$

91 Laguna de la Nevada (ca. $50^{\circ} 20^{\prime} \mathrm{S}-72^{\circ} 18^{\prime} \mathrm{W}$ )

96 Laguna del Blanquillo (ca. $50^{\circ} 20^{\prime} \mathrm{S}-72^{\circ} 18^{\prime} \mathrm{W}$ )

61 Laguna del Desierto (490’ S - $72^{\circ} 53^{\prime} \mathrm{W}$ ) Laguna del Pescado (not localized)

17 Laguna del Sello (ca. $46^{\circ} 50^{\prime} \mathrm{S}-71^{\circ} 20^{\prime} \mathrm{W}$ )

124 Laguna Escondida (ca. 51 $50^{\circ} \mathrm{S}-70^{\circ} 45^{\prime} \mathrm{W}$ )

115 Laguna Isola Bella (51 $\left.38^{\circ} \mathrm{S}-70^{\circ} 14^{\prime} \mathrm{W}\right)$

65 Laguna La Leona (ca. $49^{\circ} 35^{\prime} \mathrm{S}-72^{\circ} 35^{\prime} \mathrm{W}$ )

66 Laguna Las Toscas (ca. $49^{\circ} 35^{\prime} \mathrm{S}-72^{\circ} 35^{\prime} \mathrm{W}$ )

6 Laguna Los Cisnes (ca. $46^{\circ} 29^{\prime} \mathrm{S}-70^{\circ} 53^{\prime} \mathrm{W}$ )

92 Laguna Los Escarchados $\left(50^{\circ} 24^{\prime} \mathrm{S}-71^{\circ} 33^{\prime} \mathrm{W}\right)$

126 Laguna Morro Chico (ca. 51 $55^{\prime} \mathrm{S}-71^{\circ} 30^{\prime} \mathrm{W}$ )

91 Laguna Nimez (ca. $50^{\circ} 20^{\prime} \mathrm{S}-72^{\circ} 18^{\prime} \mathrm{W}$ )

36 Laguna Roble (ca. $47^{\circ} 55^{\prime} \mathrm{S}-73^{\circ} 00^{\prime} \mathrm{W}$ )

52 Laguna Tonchi (48 $\left.37^{\prime} \mathrm{S}-70^{\circ} 42^{\prime} \mathrm{W}\right)$

112 Laguna Travesía $\left(51^{\circ} 18^{\prime} \mathrm{S}-71^{\circ} 46^{\prime} \mathrm{W}\right)$

12 Las Heras (= Colonia Las Heras) $\left(46^{\circ} 33^{\prime} \mathrm{S}\right.$ $\left.68^{\circ} 57^{\prime} \mathrm{W}\right)$

8 Los Antiguos (46 $\left.33^{\circ} \mathrm{S}-71^{\circ} 37^{\prime} \mathrm{W}\right)$

38 Los Manantiales $\left(47^{\circ} 57^{\prime} \mathrm{S}-71^{\circ} 46^{\prime} \mathrm{W}\right)$

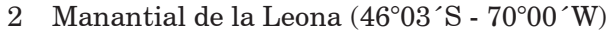

24 Mazarredo $\left(47^{\circ} 05^{\prime} \mathrm{S}-66^{\circ} 42^{\prime} \mathrm{W}\right)$

97 Meseta de las Vizcachas $\left(50^{\circ} 35^{\prime} \mathrm{S}-71^{\circ} 55^{\prime} \mathrm{W}\right)$

165 Meseta del Asador $\left(47^{\circ} 41^{\prime} \mathrm{S}-71^{\circ} 15^{\prime} \mathrm{W}\right)$

85 Meseta del Tobiano $\left(50^{\circ} 13^{\prime} \mathrm{S}-72^{\circ} 25^{\prime} \mathrm{W}\right)$

5 Meseta Lago Buenos Aires $\left(46^{\circ} 30^{\prime} \mathrm{S}\right.$ $\left.72^{\circ} 00^{`} \mathrm{~W}\right)$

51 Meseta Lago Strobel (ca. 48 $40^{\prime} \mathrm{S}-71^{\circ} 30^{\prime} \mathrm{W}$ )

70 Meseta La Siberia (ca. $50^{\circ} 00^{\prime} \mathrm{S}-73^{\circ} 00^{\prime} \mathrm{W}$ )

83 Monte León (= Ea. Monte León) (ca. 50¹4`S $68^{\circ} 55^{\prime} \mathrm{W}$ )

31 Monte Loayza (ca. $47^{\circ} 10^{\prime} \mathrm{S}-66^{\circ} 00^{\prime} \mathrm{W}$ )

109 Monte Tigre $\left(51^{\circ} 20^{\prime} \mathrm{S}-69^{\circ} 05^{\prime} \mathrm{W}\right)$

30 Monumento Natural Bosques Petrificados $\left(47^{\circ} 40^{\prime} \mathrm{S}-68^{\circ} 00^{\prime} \mathrm{W}\right)$

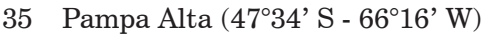

104 Paraje Le Marchand (5050`S - 69 $\left.25^{\circ} \mathrm{W}\right)$

126 Paraje Punta del Monte (ca. $51^{\circ} 37^{\prime} \mathrm{S}-71^{\circ} 17^{\prime} \mathrm{W}$ )

70 Parque Nacional Los Glaciares (ca. 5000’S $\left.73^{\circ} 00^{\prime} \mathrm{W}\right)$

36 Parque Nacional Perito Moreno (ca. $47^{\circ} 55^{\prime} \mathrm{S}$. $\left.72^{\circ} 15^{\prime} \mathrm{W}\right)$

73 Paso Biggieri $\left(50^{\circ} 09^{\prime} \mathrm{S}-72^{\circ} 00^{\prime} \mathrm{W}\right)$

76 Paso Ibáñez (ca. $50^{\circ} 10^{\prime} \mathrm{S}-70^{\circ} 00^{\prime} \mathrm{W}$ )

63 Península San Julián (ca. $49^{\circ} 18^{\prime} \mathrm{S}-67^{\circ} 43^{\prime} \mathrm{W}$ )

9 Perito Moreno (ca. $46^{\circ} 35^{\prime} \mathrm{S}-70^{\circ} 55^{\prime} \mathrm{W}$ )

81 Pico Quebrado (50 $10^{\prime} \mathrm{S}-68^{\circ} 38^{\prime} \mathrm{W}$ )

16 Pico Truncado $\left(46^{\circ} 47^{\prime} \mathrm{S}-67^{\circ} 59^{\prime} \mathrm{W}\right)$

13 Piedra Clavada (46 $\left.36^{\circ} \mathrm{S}-68^{\circ} 32^{\prime} \mathrm{W}\right)$
154 Puente Blanco (51 $\left.53^{\circ} \mathrm{S}-71^{\circ} 35^{\prime} \mathrm{W}\right)$

84 Puerto Bandera (ca. $50^{\circ} 18^{\prime} \mathrm{S}-72^{\circ} 48^{\prime} \mathrm{W}$ )

106 Puerto Coig (505 $\left.57^{\prime} \mathrm{S}-69^{\circ} 12^{\prime} \mathrm{W}\right)$

41 Puerto Deseado $\left(47^{\circ} 45^{\prime} \mathrm{S}-65^{\circ} 54^{\prime} \mathrm{W}\right)$

63 Puerto San Julián (ca. $49^{\circ} 18^{\prime} \mathrm{S}-67^{\circ} 43^{`} \mathrm{~W}$ )

84 Punta Bandera (50 $\left.18^{\prime} \mathrm{S}-72^{\circ} 48^{\prime} \mathrm{W}\right)$

43 Punta Buque (ca. $48^{\circ} 06^{\prime} \mathrm{S}-66^{\circ} 00^{\prime} \mathrm{W}$ )

117 Punta Bustamante (51 $\left.35^{\circ} \mathrm{S}-68^{\circ} 59^{\prime} \mathrm{W}\right)$

23 Punta de los Pájaros (ca. 46 $53^{\prime} \mathrm{S}-66^{\circ} 57^{\prime} \mathrm{W}$ )

135 Punta Dungeness (52 $2^{\circ} 3^{\prime} \mathrm{S}-68^{\circ} 25^{\prime} \mathrm{W}$ )

119 Punta Loyola (ca. 51 $38^{\circ} \mathrm{S}-69^{\circ} 05^{\prime} \mathrm{W}$ )

4 Punta Maqueda (46 $\left.02^{\prime} \mathrm{S}-67^{\circ} 35^{\prime} \mathrm{W}\right)$

43 Punta Médano Negro (48 $\left.01^{\prime} \mathrm{S}-65^{\circ} 57^{\prime} \mathrm{W}\right)$

44 Punta Medanosa (= Puerto Medanoso) $\left(48^{\circ} 06^{\prime} \mathrm{S}\right.$ $-65^{\circ} 55^{\prime} \mathrm{W}$ )

15 Punta Murphy $\left(46^{\circ} 38^{\prime} \mathrm{S}-67^{\circ} 17^{\prime} \mathrm{W}\right)$

81 Punta Quilla (ca. $50^{\circ} 01^{\prime} \mathrm{S}-68^{\circ} 31^{\prime} \mathrm{W}$ )

92 Reserva de Vida Silvestre Los Escarchados (ca. $50^{\circ} 24^{\prime} \mathrm{S}-71^{\circ} 33^{\prime} \mathrm{W}$ )

63 Reserva Provincial Península San Julián (ca. $\left.49^{\circ} 18^{\prime} \mathrm{S}-67^{\circ} 43^{\prime} \mathrm{W}\right)$

106 Ría Coig $\left(50^{\circ} 57^{\prime} \mathrm{S}-69^{\circ} 10^{\prime} \mathrm{W}\right)$

41 Ría Deseado $\left(47^{\circ} 45^{\prime} \mathrm{S}-65^{\circ} 54^{\prime} \mathrm{W}\right)$

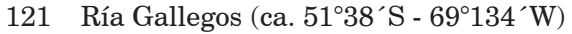

63 Ría San Julián (ca. $49^{\circ} 20^{\prime} \mathrm{S}$ - $67^{\circ} 43^{\prime} \mathrm{W}$ )

81 Ría Santa Cruz (ca. $50^{\circ} 01^{\prime} \mathrm{S}-68^{\circ} 31^{\prime} \mathrm{W}$ )

40 Río Belgrano (48 $\left.{ }^{\circ} 15^{\prime} \mathrm{S}-71^{\circ} 30^{\prime} \mathrm{W}\right)$

166 Río Blanco (ca. $49^{\circ} 30^{\prime} \mathrm{S}-72^{\circ} 50^{\prime} \mathrm{W}$ )

86 Río Bote $\left(50^{\circ} 16^{\prime} \mathrm{S}-71^{\circ} 44^{\prime} \mathrm{W}\right)$

78 Río Chico (49 $\left.56^{\prime} \mathrm{S}-68^{\circ} 32^{\prime} \mathrm{W}\right)$

106 Río Coig (= Río Coyle) $\left(50^{\circ} 58^{\prime} \mathrm{S}-69^{\circ} 11^{\prime} \mathrm{W}\right)$

166 Río de las Vueltas $\left(49^{\circ} 30^{\prime} \mathrm{S}-72^{\circ} 50^{\prime} \mathrm{W}\right)$

41 Río Deseado $\left(47^{\circ} 45^{\prime} \mathrm{S}-65^{\circ} 54^{\prime} \mathrm{W}\right)$

18 Río Ecker $\left(47^{\circ} 04^{\prime} \mathrm{S}-70^{\circ} 45^{\prime} \mathrm{W}\right)$

102 Río Frías (ca. 5050`S - $71^{\circ} 20^{\prime} \mathrm{W}$ )

121 Río Gallegos (51 $\left.38^{\circ} \mathrm{S}-69^{\circ} 13^{\prime} \mathrm{W}\right)$

72 Río Guanaco $\left(49^{\circ} 47^{\prime} \mathrm{S}-72^{\circ} 09^{\prime} \mathrm{W}\right)$

85 Río La Leona (ca. $50^{\circ} 13^{\prime} \mathrm{S}-72^{\circ} 25^{\prime} \mathrm{W}$ )

50 Río Mayer $\left(48^{\circ} 29^{\prime} \mathrm{S}-72^{\circ} 36^{\prime} \mathrm{W}\right)$

90 Río Mitre $\left(50^{\circ} 26^{\prime} \mathrm{S}-72^{\circ} 44^{\prime} \mathrm{W}\right)$

29 Río Olnie $\left(47^{\circ} 45^{\prime} \mathrm{S}-71^{\circ} 30^{\prime} \mathrm{W}\right)$

26 Río Oro $\left(47^{\circ} 26^{\prime} \mathrm{S}-71^{\circ} 57^{\prime} \mathrm{W}\right)$

10 Río Pinturas $\left(46^{\circ} 35^{\prime} \mathrm{S}-70^{\circ} 18^{\prime} \mathrm{W}\right)$

39 Río Robles $\left(47^{\circ} 57^{\prime} \mathrm{S}-71^{\circ} 51^{\prime} \mathrm{W}\right)$

127 Río Rubens $\left(52^{\circ} 00^{\prime} \mathrm{S}-71^{\circ} 50^{\prime} \mathrm{W}\right)$

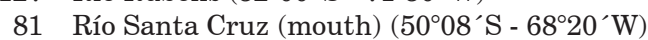

110 Río Turbio $\left(51^{\circ} 32^{\prime} \mathrm{S}-72^{\circ} 18^{\prime} \mathrm{W}\right)$

110 Rospentek (ca. 51 $32^{\prime} \mathrm{S}-72^{\circ} 18^{\prime} \mathrm{W}$ )

79 San Francisco de Paula $\left(49^{\circ} 44^{\prime} \mathrm{S}-67^{\circ} 43^{\prime} \mathrm{W}\right)$

63 San Julián $\left(49^{\circ} 18^{\prime} \mathrm{S}-67^{\circ} 43^{\prime} \mathrm{W}\right)$

81 Santa Cruz (= Puerto Santa Cruz) (50 $01^{\prime} \mathrm{S}$ $68^{\circ} 31^{\prime} \mathrm{W}$

70 Sección Centro del Parque Nacional Los Glaciares (ca 49 $50^{\prime} \mathrm{S}-73^{\circ} 00^{\prime} \mathrm{W}$ )

99 Sierra Baguales (ca. $50^{\circ} 30^{\prime} \mathrm{S}-72^{\circ} 30^{\prime} \mathrm{W}$ )

47 Tamel Aike (ca. $48^{\circ} 20^{\prime} \mathrm{S}-70^{\circ} 55^{\prime} \mathrm{W}$ )

107 Tapi Aike (= Ea. Tapi Aike) (ca. 51 $\left.{ }^{\circ} 05^{\prime} \mathrm{S}-71^{\circ} 30^{\prime} \mathrm{W}\right)$

41 Tellier (ca. $47^{\circ} 45^{\prime} \mathrm{S}-65^{\circ} 54^{\prime} \mathrm{W}$ )

167 Tres Cerros $\left(48^{\circ} 12^{\prime} \mathrm{S}-67^{\circ} 47^{\prime} \mathrm{W}\right)$

67 Tres Lagos $\left(49^{\circ} 37^{\prime} \mathrm{S}-71^{\circ} 30^{\prime} \mathrm{W}\right)$

136 Ventisquero Onelli (ca. $50^{\circ} 10^{\prime} \mathrm{S}-73^{\circ} 05^{\prime} \mathrm{W}$ ) 


\section{BIBLIOGRAPHY}

Administración de Parques Nacionales. (Inédito). Resúmenes de avistajes de fauna 1994-97, Delegación Regional Patagonia.

Albrieu, C. \& J.L Navarro. 1997. Localización y tamaño poblacional de cormoraneras en la ría Deseado (Santa Cruz, Argentina). Hornero 14 (4): 243-246.

Anónimo. 1924. Donaciones de aves, nidos y huevos. Hornero 3 (3): 291.

Anónimo. 1933. Huevos de aves de Tierra del Fuego. Hornero 5 (2): 228-230.

Anónimo. 1994. Winter secret revealed. World Birdwatch 16(3): 3

Arrighi, A.C. \& J.L Navarro.1998. Ecología reproductiva del Cormorán Imperial (Phalacrocorax atriceps) en isla Deseada (Santa Cruz, Argentina). Hornero 15 (1): 64-67.

Babarskas, M. \& J.C. Chebez. 1999. Notas breves sobre aves de la Argentina y países vecinos. Nuestras Aves $\mathrm{N}^{\circ} 39$ : 12-14.

Bala, L.O., M.A. Hernández \& M.C. Baarck. 1998. Análisis de la dieta de Limosa haemastica en Punta Loyola, Santa Cruz. X Reunión Argentina de Ornitología. Libro de resúmenes: 3.

Beck, R.H. 1912. M.S., Beck's Journal of the Brewster - Sanford South American Expedition. Unpublished (Amer. Mus. Nat. Hist.).

Beltrán, J., C. Bertonatti, A. Johnson, A. Serret \& P. Sutton. 1992. Actualizaciones sobre la distribución, biología y estado de conservación del Macá Tobiano (Podiceps gallardoi). Hornero 13 (3): 193-199.

Birabén, M. \& M.I. Hylton Scott. 1939. Observaciones sobre el "pingüino" Spheniscus magellanicus (J.R. Forster). Physis 16: 245-261.

Bó, N.A. 1956. Observaciones morfológicas y etológicas sobre el Biguá. Hornero 10 (2): 147-157.

Bohl, W.H. 1970. A study of the Crested Tinamous of Argentina. Bur. Sport Fish. Wildl., Spec. Sci. Rept. Wildl. 131: 1-101.

Bornschein, M.R. 1996. Extralimital record of the Spotwinged Falconet Spiziapteryx circumcinctus. Bulletin British Ornithological Club 116: 197-198.

Brodkorb, P. 1928. Geographic variation in Thinocorus orbignyanus Geoffroy and Lesson. Auk 45 (4): 499-500.

- 1934. Geographical variation in Belonopterus chilensis (Molina). Occasional Papers of the Museum of Zoology University Michigan 293: 1-13.

Burmeister, C.V. 1888. Relación de un viaje a la gobernación del Chubut. Anales del Museo Nacional de Buenos Aires 3: 175-251.

- 1890. Expedición a Patagonia. Anales del Museo Nacional de Buenos Aires 3: 253-321.

Cabrera, A.L. 1971. Fitogeografía de la República Argentina. Boletín de la Sociedad Argentina de Botánica 14 (1-2): 1-42.

Cabrera, A.L. \& A. Willink. 1973. Biogeografía de América Latina. Organización de Estados Americanos, Serie de Biología. Monografía № 13, 117 p.

Cadierno, S.A. \& C.D. Amorós. 1999. Cauquén Colorado (Chloephaga rubidiceps) y Cabecitanegra Austral (Carduelis barbata) en la Estancia Cóndor, provincia de Santa Cruz, Argentina. Nuestras Aves $\mathrm{N}^{\circ}$ 40: 16.
Carrara, I.S. 1952. Lobos marinos, pingüinos y guaneras de las costas del litoral marítimo e islas adyacentes de la República Argentina. Ministerio de Educación, UNLP, Facultad de Ciencias Veterinarias (Publ. Especial), $191 \mathrm{pp}$.

Casares, J. 1935. Palmípedos argentinos. Hornero 6 (1): 1-21.

Cevasco, C.M., E. Frere \& P.A. Gandini. 2001. Intensidad de visitas como condicionante de la respuesta del Pingüino de Magallanes (Spheniscus magellanicus) al disturbio humano. Ornitología Neotropical 12 (1): 75-81.

Chapman, F.M. 1919. Descriptions of proposed new birds from Peru, Bolivia, Argentina, and Chile. Bulletin American Natural History 41: 323-333.

Chebez, J.C., N.R. Rey, M. Babarskas \& A.G. Di Giácomo.1998. Las aves de los parques nacionales de la Argentina. Monografía LOLA, 12: 126 pp.

Chebez, J. C., C. Bertonatti, A. Johnson, S. Heinonen Fortabat \& G. Gil. 1988. Notas sobre la distribución de algunas aves santacruceñas. APRONA $\mathrm{N}^{\circ} 8$ : 14-27.

Coconier, E. 2005. Aves Acuáticas en la Argentina. Reporte Final, Aves Argentinas-Wetlands International, 137 pp. www.avesargentinas.org.ar

Conover, B. 1950. A study of the elegant tinamous genus Eudromia. Fieldiana, Zoology, 31: 363-374.

Cory, C.B. 1915. Notes on South American birds, with descriptions of new subspecies. Field Museum of Natural History, Ornithological Series, 1 (9): 301-335.

Cruz, I., F. Astete, G. Nauto \& L. Borrero. 2005. La colonia de nidificación de Spheniscus magellanicus de la Reserva Provincial Cabo Vírgenes (Santa Cruz, Argentina) a lo largo del tiempo: aportes de la Arqueología y la Historia. XI Reunión Argentina de Ornitología. Libro de resúmenes: 84.

Cunningham, R.O. 1871. Notes on the Natural History of the Straits of Magellan and West coast of Patagonia, made during the Voyage of H. M. S. "Nassau", 1866-1869. Edinburgh.

Dabbene, R. 1910. Ornitología Argentina. Anales del Museo Nacional Buenos Aires, Serie 3 Tomo 18: 1-513.

- 1913. Distribution des oiseaux en Argentine d'après l'ouvrage de Lord Brabourne et Chubb The Birds of South America (pt. 1). Physis 1: 241-261.

- 1920a. Notas sobre los chorlos de Norteamérica que invernan en la República Argentina. Hornero 2 (2): 99-128.

- 1920b. Miscelanea ornitológica. III El huevo de la perdiz Tinamotis ingoufi Oust. IV Sobre nidificación del flamenco, Phoenicopterus chilensis Mol. Hornero 2 (2): 133-136.

- 1923. La martineta (Calopezus elegans) en Santa Cruz (Patagonia). Hornero 3 (2): 195.

Daciuk, J. 1976a. Notas faunísticas y bioecológicas de Península Valdés y Patagonia XV. Estudio bioecológico inicial de los Espheniscidos visitantes y colonizadores de Península Valdés y costas aledañas (Prov. de Chubut, Argentina). Physis, Sec. C 35 (90): 43-56. 1976b. Notas faunísticas y bioecológicas de Península Valdés y Patagonia. XIX. Pingüinos que nidifican y arriban en sus migraciones a las costas de Santa Cruz e Islas Malvinas (Aves, Spheniscidae). Neotrópica 22 (68): 87-92. 
- 1977. Notas faunísticas y bioecológicas de península Valdés y Patagonia. VI. Observaciones sobre áreas de nidificación de la avifauna del litoral marítimo patagónico (provincias de Chubut y Santa Cruz, Rep. Argentina). Hornero 11 (5): 361- 376.

Daneri, C.A. 1959. La nidificación del biguá Phalacrocorax olivaceus olivaceus (Humboldt) en Puerto Deseado. Physis 21 (61): 273-277.

Darrieu, C.A. 1980. Las razas geográficas de Cyanoliseus patagonus (Aves Psittacidae). Neotrópica 26 (76): 207-216.

- 1982. Contribución al conocimiento de las razas de Enicognathus ferrugineus (Müller) (Aves, Psittacidae). Historia Natural 2 (12): 93-100.

De la Peña, M.R. 1980. Notas nidológicas sobre biguáes y cormoranes (Aves: Anhingidae y Phalacrocoracidae). Historia Natural 1 (16): 109-112.

- 1987. Nidos y huevos de aves argentinas. Ed. del autor, $260 \mathrm{pp}$.

- 2005. Reproducción de las aves argentinas (con descripción de pichones). L.O.L.A., Monografía 20, $845 \mathrm{pp}$.

De Lucca, E.R. 1992. Métodos y éxito de caza de una hembra de Halconcito Colorado (Falco sparverius) en la Patagonia argentina. Nuestras Aves $\mathrm{N}^{\circ}$ 26: 25-26.

- $\quad 1993 a$. Un caso de poliginia en el Halconcito Colorado (Falco sparverius). Hornero 13 (4): 299-302.

- 1993b. Nidificación del Halconcito Colorado (Falco sparverius) en la Patagonia. Hornero 13 (4): 302-305.

De Lucca, E.R. \& M.D. Saggese. 1992. Aves del departamento Deseado, Santa Cruz. Hornero 13 (3): 259-260.

- 1993. Biología Reproductiva de Geranoaetus melanoleucus. Primera Reunión de Ornitología de la Cuenca del Plata: Libro de Resúmenes 15.

- $\quad$ 1995. Fratricidio en el Aguila Mora Geranoaetus melanoleucus. Hornero 14 (1 y 2): 38-39.

De Lucca, E.R., M.D. Saggese \& M. Ióppolo. 1993. Proyecto Aguila Escudada. Campaña 1987-1988. Primera Reunión de Ornito-logía de la Cuenca del Plata: Libro de Resúmenes 14.

Del Hoyo, J., A. Elliott, \& J. Sargatal. 1992. Handbook of the birds of the world. Vol. 1. Ostrich to Ducks. Lynx Edicions, Barcelona.

- 1994. Handbook of the birds of the world. Vol. 2. New World Vultures to Guineafowl. Lynx Edicions, Barcelona.

- 1996. Handbook of the birds of the world. Vol. 3. Hoatzin to Auks. Lynx Edicions, Barcelona.

- 1997. Handbook of the birds of the world. Vol. 4. Sandgrouse to Cuckoos. Lynx Edicions, Barcelona.

- 1999. Handbook of the birds of the world. Vol. 5. Barn-owls to Hummingbirds. Lynx Edicions, Barcelona.

- 2001. Handbook of the birds of the world. Vol. 6 . Mousebirds to Hornbills. Lynx Edicions, Barcelona. 2002. Handbook of the birds of the world. Vol. 7. Jacamars to Woodpeckers. Lynx Edicions, Barcelona.

Di Giácomo, A.S., V. De Francesco \& E.G. Coconier. 2005. Areas importantes para la conservación de las aves en la Argentina. Aves Argentinas. CD.

Doello Jurado, M. 1917. Sobre aves de Puerto Deseado. Hornero 1 (1): 8-16.

Earnshaw, A. \& N. Earnshaw. 2000. www.geocities.com, Lista de aves avistadas en un viaje a la Patagonia- enero 2000. Available from: http://viajesnat/ patagonia2000/p2000-li.htm.

Edelaar, P., M. Abril \& J. Torres Dowdall. 2005. Primer registro para agachonas (Familia Thinocoridae) bebiendo en estado silvestre. XI Reunión Argentina de Ornitología. Libro de resúmenes: 93.

Erize, F. 1983. Observaciones sobre el Macá Tobiano. Hornero $\mathrm{N}^{\circ}$ extraordinario: 256-268.

Ferrari, S. \& C. Albrieu. 2005. Estrategias y acciones para la conservación de las aves playeras en el estuario del río Gallegos, Santa Cruz. XI Reunión Argentina de Ornitología. Libro de resúmenes: 40.

Ferrari, S., B. Alegre \& P. Gandini. 2004. Dieta del Cormorán Imperial (Phalacrocorax atriceps) en el sur de Santa Cruz (Patagonia, Argentina). Ornitología Neotropical 15 (1): 103-110.

Ferrari, S., S. Imberti \& C. Albrieu. 2003. Magellanic Plovers Pluvianellus socialis in southern Santa Cruz Province, Argentina. Wader Study Group Bulletin 101/102: 70-76.

Ferrari, S., Z. Lizarralde, C. Albrieu \& S. Pittaluga. 2005. El Playero Rojizo (Calidris canutus rufa) en el sur de Santa Cruz: abundancia, patrón migratorio y alimentación. XI Reunión Argentina de Ornitología. Libro de resúmenes: 96.

Fjeldså, J. 1985. Origin, evolution, and status of the avifauna of Andean wetlands. Neotropical Ornithology, Ornithological Monographs $\mathrm{N}^{\circ}$ 36: 85-112.

-1986. Feeding ecology and possible life history tantics of the Hooded Grebe Podiceps gallardoi. Ardea 74: 40-58.

Fjeldså, J. \& N. Krabbe. 1990. Birds of the High Andes. Zoological Museum, University of Copenhagen, Denmark.

Frere, E. \& P.A. Gandini. 1998. Distribución reproductiva y abundancia de las aves marinas de Santa Cruz. Parte II: de Bahía Laura a Punta Dungeness. Pp. 153-177 En: P. Yorio, E. Frere, P. Gandini \& G. Harris (eds) Atlas de la disribución reproductiva de aves marinas en litoral patagónico argentino. Fundación Patagonia Natural y Wildlife Conservation Society, Buenos Aires.

- 2001. Aspects of the breeding biology of the Redlegged Cormorant Phalacrocorax gaimardi on the Atlantic coast of South America. Marine Ornitology 29 (2): 67-70.

Frere, E., P.A. Gandini \& P.D. Boersma. 1996. Aspectos particulares de la biología de reproducción y tendencia poblacional del Pingüino de Magallanes (Spheniscus magellanicus) en la colonia de Cabo Vírgenes, Santa Cruz, Argentina. Hornero 14 (3): 50-59.

- 1998. Breeding ecology of the Magellanic Penguin at Cabo Vírgenes, Argentina: What determine reproductive success? Colonial Waterbirds 21: 205-210.

Frere, E., P.A. Gandini \& T.M. Holik. 1992. Nidificación de Theristicus melanopis en la provincia de Santa Cruz. Hornero 13 (3): 247-248.

Frere, E., P.A. Gandini \& V. Lichtschein. 1996. Variación latitudinal en la dieta del Pingüino de Magallanes (Spheniscus magellanicus) en la costa patagónica, Argentina. Ornitologia Neotropical 7: 35-41.

Frere, E., P.A. Gandini \& R. Martínez Peck. 2000. Gaviota Cocinera (Larus dominicanus) como vector potencial de patógenos, en la costa patagónica. Hornero 15 (2): 93-97. 
Frere, E., M. Gandini, P.A. Gandini, T. Holik, V. Lichtschein \& M. Oliva Day. 1993. Variación anual en el número de adultos reproductivos en una nueva colonia de Pingüino Penacho Amarillo Eudyptes crestatus (Spheniscidae) en Isla Pingüino (Santa Cruz, Argentina). Hornero 13 (4): 293-294.

Frere, E., F. Quintana \& P. Gandini. 2005. Cormoranes de la costa patagónica: estado poblacional, ecología y conservación. Hornero 20 (1): 35-52.

Gandini, P. \& E. Frere. 1995. Distribución, abundancia y ciclo reproductivo del Cormorán Gris Phalacrocorax gaimardi en la costa patagónica, Argentina. Hornero 14 (1 y 2): 57-60.

- 1998a. Distribución reproductiva y abundancia de las aves marinas de Santa Cruz. Parte I: de La Lobería a Islote del Cabo. En: P. Yorio, E. Frere, P. Gandini \& G. Harris (eds). Atlas de la disribución reproductiva de aves marinas en litoral patagónico argentino. pp. 119-151. Fundación Patagonia Natural y Wildlife Conservation Society, Buenos Aires. 1998b. Seabird and shorebird diversity and associated conservation problems in Puerto Deseado, Patagonia, Argentina. Ornitologia Neotropical 9: 13-22.

Gandini, P., E. Frere \& D. Boersma. 1997. Efectos de la calidad de habitat sobre el éxito reproductivo del Pingüino de Magallanes (Spheniscus magellanicus) en Cabo Vírgenes, Santa Cruz, Argentina. Ornitologia Neotropical 8 (1): 37-48.

Gandini, P.A., E. Frere \& T.M. Holik. 1992. Implicancias de las diferencias en el tamaño corporal entre colonias para el uso de medidas morfométricas como método de sexado en Spheniscus magellanicus. Hornero 13 (3): 211-213.

Gandini, P., E. Frere \& F. Quintana. 2005. Feeding performance and foraging area of the Red-legged Cormorant. Waterbirds 28 (1): 41-45.

Goodall, R.N., A.C.M. Schiavini \& M. Galussio. 1991. Summer evaluation of mammals and birds of the Southwestern South Atlantic coast, between Cabo Vírgenes and Cabo San Sebastián. Informe inédito, Total Austral S.A., 182 pp.

Gould, J. 1837a. Observations on the Raptorial Birds in Darwin's Collection, with characters of the New Species. Proceedings of the Zoological Society of London 1837: 9-11.

- 1837b. On a new Rhea (Rhea Darwinii) from Mr. Darwin's Collection. Proceedings of the Zoological Socciety of London 1837: 35-36.

Gould, J. \& C. Darwin. 1838-1841. Birds. En C. Darwin, Zoology of H.M.S. Beagle. 1832 to 1836, vol. 1. Smith, Elder \& Co, London, $164 \mathrm{pp}$.

Gray, G.R. 1844. List of the species of birds in the British Museum 3: 1-209.

Gurney, J.H. 1882. Notes on a "Catalogue of the Accipitres in the British Museum" by R. Bowdler Sharpe (1874). Ibis 1882: 290-321.

Hartert, E. \& S. Venturi. 1909. Notes sur les oiseaux de la République Argentina. Novitates Zoology 16: 159-267.

Hellmayr, C.E. 1932. The birds of Chile. Field Museum of Natural History, Zoological Series 19: 1-472.

Hellmayr, C.E. \& B. Conover. 1942. Catalogue of birds of the Americas and the adjacent islands. Field
Museum of Natural History, Vol. 13 Part 1 (1) (Rheidae to Columbidae): 1-636.

1948a. Catalogue of birds of the Americas and the adjacent islands. Field Museum of Natural History, Vol. 13, Part 1 (2) (Spheniscidae to Anatidae): 1-434. 1948b. Catalogue of birds of the Americas and the adjacent islands. Field Museum of Natural History, Vol. 13, Part 1 (3) (Jacanidae to Alcidae): 1-383.

- 1949. Catalogue of birds of the Americas and the adjacent islands. Field Museum of Natural History, Vol. 13, Part 1 (4) (Cathartidae to Falconidae): 1-358.

Hernández, M.A., V.L. D’Amico \& L.O. Bala. 2004. Presas consumidas por el Playero Rojizo (Calidris canutus) en Bahía San Julián, Santa Cruz, Argentina. Hornero 19 (1): 7-11.

Humphrey, P.S. \& D. Bridge. 1970. Apuntes sobre distribución de aves en la Tierra del Fuego y la Patagonia argentina. Revista del Museo Argentino de Ciencias Naturales Bernardino Rivadavia, NS, Zoologia 10 (17): 251-265.

Humphrey, P.S. \& B.C. Livezey. 1982. Molts and plumages of Flying Steamer-Ducks (Tachyeres patachonicus). Occasional Papers of the Museum of Natural History University Kansas N ${ }^{\circ}$ 103: 1-30.

Iglesias, G.J. \& A.A. Pérez. 1998. Región 4: Patagonia. En: P. Canevari, D.E. Blanco, E.H. Bucher, G. Castro \& I. Davidson (eds.). 1998. Los Humedales de la Argentina: Clasificación, Situación Actual, Conservación y Legislación. Wetlands International Publ. 46, Buenos Aires, Argentina. 208 pp.

Imberti, S. 2001. Primera observación del Ñacundá (Podager nacunda) en Santa Cruz, Argentina, la más austral para la especie. Nuestras Aves $\mathrm{N}^{\circ}$ 41: 10 . 2003. Notes on the distribution and natural history of some birds in Santa Cruz and Tierra del Fuego provinces, Patagonia, Argentina. Cotinga 19: 15-24. 2005. Aves de Los Glaciares: Inventario Ornitológico del Parque Nacional Los Glaciares, Santa Cruz, Patagonia, Argentina. Aves Argentinas \& APN.

- Lista de aves de la Reserva Provincial Cabo Vírgenes. (Informe inédito/ Unpublished report a), 7 pp.

- Lista de aves zona Estancia La Angostura. (Informe inédito/Unpublished report b), 3 pp.

Imberti, S. \& C. Albrieu. 2001. Lista de las aves de Laguna Nimez. Universidad Nacional de la Patagonia Austral 14 pp.

Imberti, S., S.M. Sturzenbaum \& M. McNamara. 2004. Actualización de la distribución invernal del Macá Tobiano (Podiceps gallardoi) y notas sobre su problemática de conservación. Hornero 19 (2): 83-89. Jardine, W. \& P.J. Selby. 1830. Illustrations of Ornithology, 3, part 7, $131 \mathrm{pl}$.

Jehl, J.R. Jr. 1975. Pluvianellus socialis: biology, ecology, and relationships of an enigmatic Patagonian shorebirds. Transactions, San Diego Society of Natural History 18: 29-73.

- 1978. A new hybrid oystercatcher from South America, Haematopus leucopodus $x$ H. ater. Condor 80 (3): 344-346.

Jehl, J.R. Jr. \& M.A.E. Rumboll. 1976. Notes on the avifauna of Isla Grande and Patagonia, Argentina. Transactions, San Diego Society of Natural History 18: $145-154$ 
Junge, G.C.A. \& K.H. Voous. 1955. The distribution and the relationship of Sterna eurygnatha Saunders. Ardea 43: 226-247.

König, C. 1991. Zur Taxonomie und Ökologie der Sperlingskäuze (Glaucidium spp.) des Andenraumes. Ökologie Vögel 13: 15-76.

Lange, C. 1981. Una temporada de observaciones sobre Podiceps gallardoi (Aves: Podicipediformes). Ecología y etología. Neotrópica 27 (77): 39-56.

Laredo, C.D. 1988. Sobre recuperaciones de Chloephaga picta. En Capllonch, P. Sobre el anillado de aves en Argentina. Serie Monográfica y Didáctica $\mathrm{N}^{\circ} 1$. Universidad Nacional de Tucumán, Facultad de Ciencias Naturales e Instituto Miguel Lillo, 32 pp.

Liebermann, J. 1936. Monografía de las Tinamiformes argentinas y en problema de su domesticación. Edición del autor, Buenos Aires, 99 pp.

Livezey, B.C. \& P.S. Humphrey. 1983. Mechanics of steaming in Steamer-Ducks. Auk 100 (2): 485-488.

Lucero, M.M. 1982. El anillado de aves en la República Argentina. Fundación Miguel Lillo Miscelania № 74: 5-35.

Lucero, M.M. 1987. El anillado de aves en Argentina 1961-1986, Undécimo informe. Neotrópica 33 (90): 133-138.

Márquez, F., L. Pozzi, J. Lancelotti, M. Pascual \& P.Yorio. 2005. Ensambles de aves acuáticas del sistema de lagunas del Lago Strobel, Santa Cruz: datos preliminares. XI Reunión Argentina de Ornitología. Libro de resúmenes: 126.

Martens, G.H. 1900. Hamburger Magahaensische Sammeheise: Vögel. Naturhistorischen l'Museum zu Hamburg 5 (6): 2-34.

Martín, C. 1992. Macá Tobiano en un Parque Nacional. Nuestras Aves № 27: 34.

Mazar Barnett, J. 2001. Nuevo registro del picaflor andino (Oreotrochilus leucopleurus) para Santa Cruz (Argentina). Nuestras Aves $\mathrm{N}^{\circ}$ 41: 31.

Mazar Barnett, J. \& M. Pearman. 2001. Lista comentada de las aves argentinas. Lynx Edicions, 164 pp.

Mazar Barnett, J., M. della Seta, S. Imberti \& G. Pugnali. 1998. Notes on the rediscovery of the Austral Rail Rallus antarcticus in Santa Cruz, Argentina. Cotinga 10: 96-101.

Miller, E.H. \& A.J. Baker. 1980. Displays of the Magellanic Oystercatcher (Haematopus leucopodus). Wilson Bulletin 92 (2): 149-168.

Millones A., E. Frere \& P. Gandini. 2005. Dieta del Cormorán Gris (Phalacrocorax gaimardi) en la Ría Deseado, Santa Cruz, Argentina. Ornitología Neotropical 16: 519-527.

Murphy, R.C. 1916. Notes on American subantarctic cormorants. Bulletin of the American Museum of Natural History 35: 31-48.

- 1925. Notes on certain species and races of oystercatchers. American Museum Novitates 194: 5-15.

- $\quad$ 1936. Oceanic Birds of South America. 2 Vols. American Museum of Natural History, New York.

Murphy, R.C. \& F. Harper. 1921. A review of the diving petrels. Bulletin of the American Museum of Natural History 44: 495-554.

Nasca, P.B., P.A. Gandini \& E. Frere. 2004. Caracterización de las asociaciones de alimentación multiespecíficas de aves marinas en la Ría Deseado, Santa Cruz, Argentina. Hornero 19 (1): 29-36.
Navas, J.R. 1991. Fauna de agua dulce de la República Argentina. Vol. 43 Aves, Fasc. 3 Gruiformes. PROFADU. 80 pp.

Navas, J.R. \& N.A. Bó. 1981. Nuevas aportaciones a la taxionomía de las razas geográficas de Eudromia elegans y Eudromia formosa. Revista del Museo Argentino de Ciencias Naturales Bernardino Rivadavia, Zool. 11 (2): 33-59.

- 1986. Revisión de las subespecies argentinas de Vanellus chilensis (Aves, Charadriidae). Neotrópica 32 (88): 157-165.

- 1996. Notas breves sobre las especies del género Attagis en la Argentina (Aves: Thinocoridae). Neotrópica 42 (107-108): 76.

- 1997. Nuevos aportes sobre las características y la distribución geográfica de Speotyto cunicularia partridgei (Aves: Strigidae). Neotrópica 43 (109 y 110): 127-128.

Navas, J.R. \& A.R. Camperi. 1999. Los tipos de aves que se conservan en el Museo Argentino de Ciencias Naturales de Buenos Aires. Revista del Museo Argentino de Ciencias Naturales, N. S. 1 (1): 109-113.

Nuechterlein, G.L. \& R.W. Storer. 1985. Aggressive behavior and interspecific killing by Flying SteamerDucks in Argentina. Cóndor 87: 87-91.

Oates, E.W. 1901. Catalogue of the collection of birds'eggs in the British Museum. Vol. 1. Taylor \& Francis, London.

Olrog, C.C. 1948. Observaciones sobre la avifauna de Tierra del Fuego y Chile. Acta Zoológica Lilloana 5: 437-531.

- 1962. Notas ornitológicas sobre la colección del Instituto Miguel Lillo. VI. Acta Zoológica Lilloana 18: $111-120$.

- 1973. Notas ornitológicas. IX. Sobre la colección del Instituto Miguel Lillo . Acta Zoológica Lilloana 30: 7-11.

- $\quad$ 1975. El anillado de aves en la Argentina. 1961-1974. Noveno informe. Neotrópica 21 (64): 17-19.

- 1976. El anillado de aves en la Argentina. 1961-1976, décimo informe. Neotrópica 22 (68): 113-114.

Orfila, R.N. 1937. Los Psittaciformes argentinos. Hornero 6 (3): 365-382.

Oustalet, E. 1890. Description d'un nouveau tinamou de la Patagonie. Annales des Sciences Naturelles, Zoologie, Paris, Ser. 7, 9: 18.

- 1891. Misión Scientifique du Cap Horn, 1882-83. VI. Zool., Oiseaux, 341 pp.

Paynter, R.A. Jr. 1995. Ornithological gazetteer of Argentina. 2nd edition. Cambridge, Massachusetts.

Pergolani de Costa, M.J.I. 1942. Los Pícidos argentinos (carpinteros). Hornero 8(2): 155-170.

- 1955. Las "Avutardas" - Especies que dañan a los cereales y las pasturas. IDIA $\mathrm{N}^{\circ}$ 88: 1-8.

Plotnick, R. 1961. Migración de las avutardas. IDIA $\mathrm{N}^{\circ}$ 167: 18-28.

Pozzi, A. 1923. Las perdiz de Santa Cruz (Patagonia) Tinamotis ingoufi Oust. Hornero 3 (2): 180-184.

Pugnali, G., M. Pearman, G. Escudero, D. Vaquero \& T. Chater. 2004. New localities for the Austral Rail Rallus antarcticus in Argentina, and first record from the Falkland Islands. Cotinga 22: 35-37.

Reichenow, A. 1920. New forms of Riparia, Hirundo, Pachysylvia, Thinocorus. Journal für Ornithology 68: 88. 
Renard, A. 1931. Algunas observaciones sobre aves durante un viaje a la Ría de Deseado. Hornero 4 (4): 412-414.

Rumboll, M.A.E. 1974. Una nueva especie de macá (Podicipedidae). Comunicaciones del Museo Argentino de Ciencias Naturales Bernardino Rivadavia, Zoología 4 (5): 33-35.

- 1975. Notas sobre Anseriformes. El Cauquén de Cabeza Colorada (Chloephaga rubidiceps): Una nota de alarma. Hornero 11 (4): 315-316.

- 1991. Hallazgo de Anas discors en Santa Cruz. Nuestras Aves $\mathrm{N}^{\circ}$ 24: 23.

Rumboll, M.A.E. \& P.J. Canevari. 1975. Invasión de Bubulcus ibis en la Argentina (Aves: Ardeidae). Neotrópica 21 (66): 162-165.

Saggese, M.D. \& E.R. De Lucca. 1995. Reproducción del Gavilán Ceniciento Circus cinereus en la Patagonia argentina. Hornero 14 (1 y 2): 21-26.

- 2001. Biología reproductiva del Aguila Mora (Geranoaetus melanoleucus) en la Patagonia sur, Argentina. Hornero 16 (2): 77-84.

Salvadori, T. 1900. Contribuzione all' avifauna dell' America Australe (Patagonia, Terra del Fuoco, Isola Degli Stati, Isole Falkland). Annali del Museo Civico di Storia Naturale di Genova Ser. 2 (20): 609-634.

Saunders, H. 1896. Catalogue of the Gaviae in the Collection of the British Museum. Gaviae (Terns, Gulls and Skuas) Vol. 25, London.

Savigny, C., F. Firstater \& G. Caille. 2005. Observaciones de aves marinas en la plataforma continental austral argentina: registros extralimitales y especies poco documentadas. XI Reunión Argentina de Ornitología. Libro de resúmenes: 154.

Schiavini, A., P. Yorio, P. Gandini, A. Raya Rey \& P.D. Boersma. 2005. Los pingüinos de las costas argentines: estado poblacional y conservación. Hornero 20 (1): 5-23.

Sclater, P.L. \& O. Salvin. 1869. Second list of birds collected, during the survey of the Straits of Magellan, by Dr. Cunningham. Ibis 1869: 283-286.

- 1871. A revised list of the Neotropical Laridae. Proceedings of the Zoological Society of London, 1871: 564-580.

Sclater, P.L., O. Salvin \& A. Newton. 1870. Third list of birds collected, during the survey of the Straits of Magellan, by Dr. Cunningham. Ibis 1870: 499-504.

Scott, D.A \& M. Carbonell. 1986. Inventario de humedales de la Región Neotropical. (compiladores). IWRB Slimbridge UICN Cambridge.

Scott, W.E.D. \& R.B. Sharpe. 1904. Reports of the Princenton University Expeditions to Patagonia, 1896-1899. Vol. II, Ornithology, Part I, pp. 1-112.

- 1910. Reports of the Princenton University Expeditions to Patagonia, 1896-1899. Vol. II, Ornithology, Part II, pp. 113-344.

- 1912. Reports of the Princenton University Expeditions to Patagonia, 1896-1899. Vol. II, Ornithology, Part III, pp. 345-504.

- 1915. Reports of the Princenton University Expeditions to Patagonia, 1896-1899. Vol. II, Ornithology, Part IV, pp. 505-718.

Seebohm, H. 1887. The Geographical Distribution of the Charadriidae, or the Plovers, Sandpipers, Snipes, and their allies. London \& Manchester, pp. 1-524.
Serié, P. 1926. Miscelánea ornitológica. V. Captura de un "Ñacurutú" en Santa Cruz. Hornero 3 (4): 417-420. Serret, A. \& A. Johnson. 1986. Primera cita del Picaflor Serrano Ventrinegro para la provincia de Santa Cruz. Nuestras Aves $\mathrm{N}^{\circ}$ 10: 14.

Sharpe, R.B. 1896. Catalogue of the Limicolae in the Collection of the British Museum. Vol. 24. London.

Sharpe, R.B. \& W.R. Ogilvie-Grant. 1898. Catalogue of the birds in the British Museum. Vol. 26. London, $687 \mathrm{pp}$.

Steullet, A.B. \& E.A. Deautier. 1935. Catálogo Sistemático de las Aves de la República Argentina. Obra del Cincuentenario del Museo de La Plata, Universidad Nacional de La Plata, Tomo 1 (1ㄹ entrega): 1-256. 1936. Catálogo Sistemático de las Aves de la República Argentina. Obra del Cincuentenario del Museo de La Plata, Universidad Nacional de La Plata, Tomo 1 ( $2^{\mathrm{a}}$ entrega): 257-492.

- 1939. Catálogo Sistemático de las Aves de la República Argentina. Obra del Cincuentenario del Museo de La Plata, Universidad Nacional de La Plata, Tomo 1 (3 $3^{\mathbf{a}}$ entrega): 493-732.

- 1945. Catálogo Sistemático de las Aves de la República Argentina. Obra del Cincuentenario del Museo de La Plata, Universidad Nacional de La Plata, Tomo 1 (4⿳亠口了 entrega): 733-932.

Stone, W. 1915. Rep. Princeton Univ. Exp., 2 (2), pt. 4

1928. Reports of the Princeton University Expedition to Patagonia, 1896-1899. Vol. II, Ornithology, Part V, pp. 719-857.

Straneck, R. \& A. Johnson. 1984. Vocalizaciones en relación al comportamiento del Macá Tobiano (Podiceps gallardoi Rumboll). Revista del Museo Argentino de Ciencias Naturales Bernardino Rivadavia, Zoología 13 (19): 177-188.

Torres, E.R. \& F.A. Vargas. 2005. Dieta del Macá Tobiano (Podiceps gallardoi) en el estuario del río Gallegos, Santa Cruz, Argentina. Nuestras Aves $N^{\circ} 50$ : 33-55.

Vinciguerra, D. 1884. Sulla fauna dell' America australe. Bollettino della Societá Geografica Italiana, pp. 185-811.

Wetmore, A. 1926a. Report on a collection of birds made by J. R. Pemberton in Patagonia. University of California Publications in Zoology 24 (4): 395-474.

- 1926b. Observations on the birds of Argentina, Paraguay, Uruguay, and Chile. Bulletin of the United States National Museum 133: 1-448.

Wilson, R., R. Locca, J.A. Scolaro, S. Laurenti, J. Upton, H. Galleli, E. Frere \& P. Gandini. 2001. Magellanic Penguins Spheniscus magellanicus commuting through San Julian Bay: do current trends induce tidal tactics? Journal of Avian Biology 32: 83-89.

Wilson, R.P., J.A. Scolaro, D. Grémillet, M.A.M. Kierspel, S. Laurenti, J. Upton, H. Galleli, F. Quintana, E. Frere, G. Müller, M. Thor Straten \& I. Zimmer. 2005. How do Magellanic penguins cope with variability in their access to prey? Ecological Monographs 75 (3): 379-401.

Yorio, P. 1998. Región 5: zona costera patagónica. En: P. Canevari, D.E. Blanco, E.H. Bucher, G. Castro \& I. Davidson (eds.). 1998. Los Humedales de la Argentina: Clasificación, Situación Actual, Conservación y Legislación. Wetlands International Publ. 46, Buenos Aires, Argentina. 208 pp. 
- 2005. Estado poblacional y de conservación de gaviotines y esqúas que se reproducen en el litoral marítimo argentino. Hornero 20 (1): 75-93.

Yorio, P., M. Bertellotti, P. Gandini \& E. Frere. 1998. Kelp Gulls Larus dominicanus breeding on the argentine coast: population status and relationship with coastal management and conservation. Marine Ornithology 26: 11-18.

Yorio, P., M. Bertellotti \& P. García Borboroglu. 2005 Estado poblacional y de conservación de gaviotas que se reproducen en el litoral marítimo argentino. Hornero 20 (1): 53-74.

Zapata, A.R.P. 1965. Nuevas localidades de nidificación de Leucophaeus scoresbii (Traill) y Sterna sandvi- censis eurygnatha Saunders. (Aves, Laridae). Physis 25 (70): 383-385.

- 1967. Observaciones sobre aves de Puerto Deseado provincia de Santa Cruz. Hornero 10 (4): 351-378.

- 1969. Aves observadas en el Golfo San Jorge, Provincias de Chubut y Santa Cruz, Argentina. Zoología Platense 1 (5): 21-27.

Zapata, A.R.P. \& H.S. Martínez. 1972. Algunas aves no citadas y otras pocos frecuentes para el sur de la provincia de Buenos Aires. Acta Zoológica Lilloana 29: 181-199.

Zotta, A.R. \& S. Da Fonseca. 1936. Sinopsis de los Ciconiiformes Argentinos. Hornero 6 (2): 240-248.

Recibido: 24-VIII-2007

Aceptado: 25-IV-2008 